


\title{
Albert-Ludwigs-Universität Freiburg
}

\author{
Zulassungsarbeit \\ zum 1. Staatsexamen \\ für das Lehramt an Gymnasien
}

mit dem Titel:

\section{Die Darstellung des intergenerationellen Traumas im Film La teta asustada von Claudia Llosa}

\author{
vorgelegt von \\ Yasemin Soydan \\ betreuender Dozent: Prof. Dr. Hermann Herlinghaus \\ Philologische Fakultät \\ Romanisches Seminar \\ Lehrstuhl für \\ Lateinamerikanische Literaturwissenschaft
}




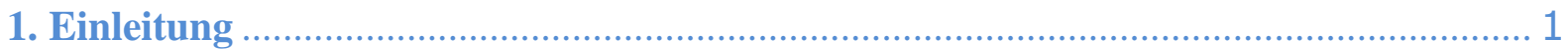

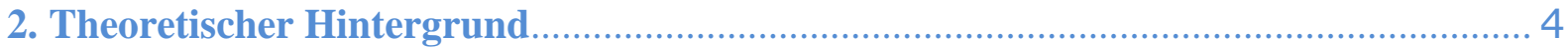

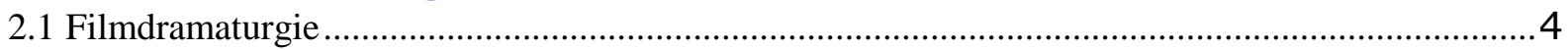

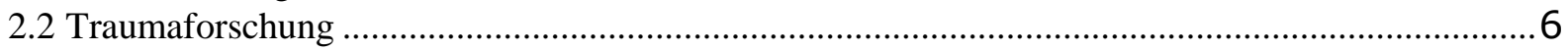

2.3 Zusammenhang zwischen Filmdramaturgie und Traumaforschung …........................................

3. Intergenerationelle Traumatisierung in Peru nach Theidon....................................... 10

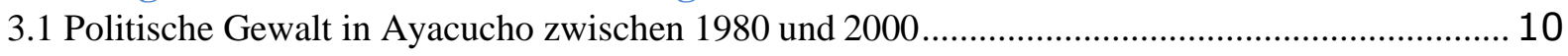

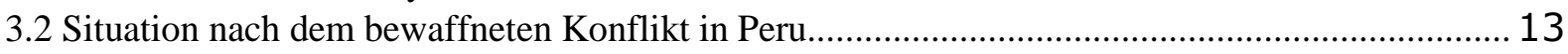

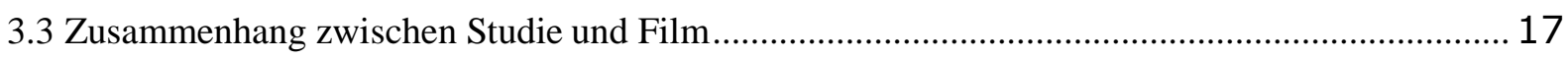

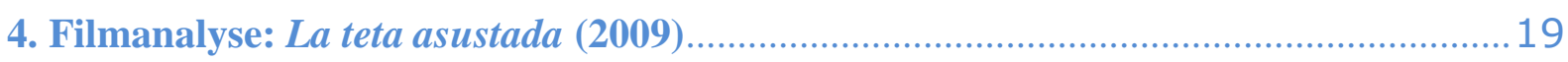

4.1 Methode

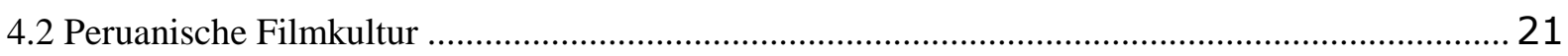

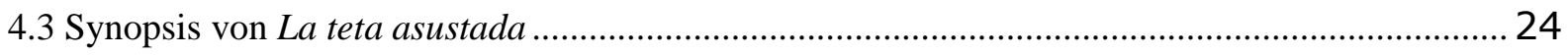

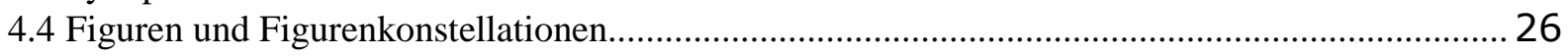

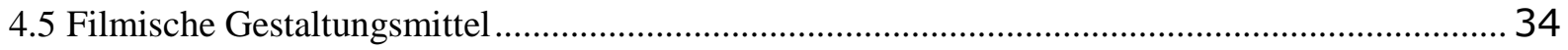

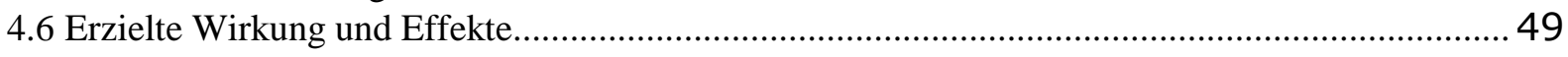

5. Abschließender Kommentar und Fazit .................................................................55

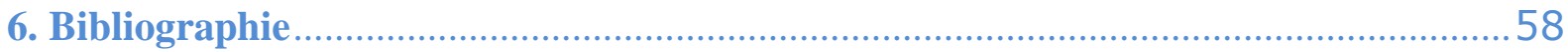

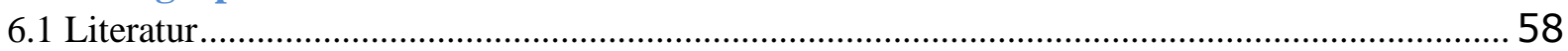

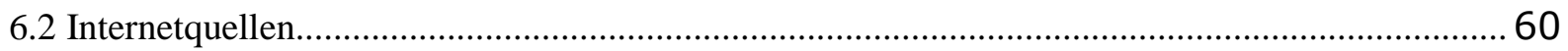

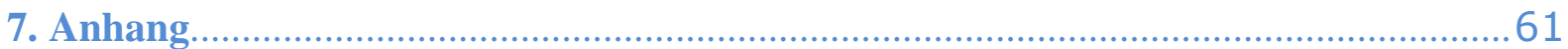

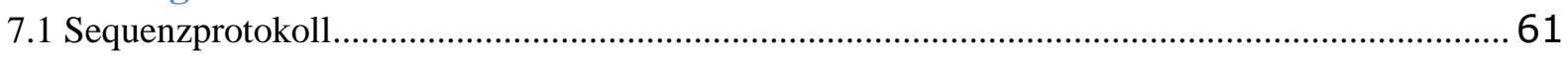

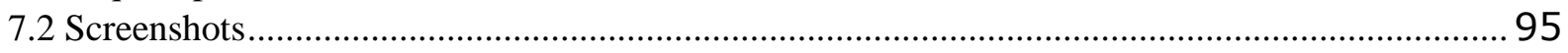




\section{Einleitung}

Medien werden genutzt, um Erinnerungen $\mathrm{zu}$ (re)konstruieren und ein historisches Bewusstsein zu schaffen. Sie erzählen die Ereignisse eines Landes oder des Lebens einer Person und erinnern an vergangene Erfahrungen. Außerdem werden Unwissende aufgeklärt. (Kino-) Filme sind ein besonders beliebtes Medium, denn sie können Vergangenes auf andere Art und Weise darstellen, als unter anderem die Politik- oder Geschichtswissenschaften. Sie können die historische Materie freier interpretieren und künstlerisch untermalen. Filme archivieren die Geschichte nicht, sondern regen zum Reflektieren an.

Südamerikanische Geschichte ist beispielsweise im Vergleich zur Geschichte der Bundesrepublik Deutschland bei weitem nicht so oft und intensiv diskutiert, sowie filmisch verarbeitet worden. Es existieren sowohl auf nationaler als auch internationaler Ebene unzählige Museen, Denkmale, Dokumentationen, Sammlungen von historischen Gegenständen, Interviews mit Zeitzeugen und Spielfilme, die über den Holocaust und dessen Folgen aufklären und die Menschheit daran hindern, zu vergessen. Kaum ein anderes Land arbeitet derart intensiv an der Aufarbeitung der eigenen Vergangenheit wie die Bundesrepublik Deutschland. Auch Menschen in südamerikanischen Staaten mussten in der näheren Vergangenheit an Unterdrückung, Entführungen und Völkermorden leiden. Doch es existieren nur wenige Dokumentationen, die an diese traumatischen Zeiten erinnern und ein kollektives Gedächtnis schaffen.

Claudia Llosa ist eine der wenigen Personen, die an der Bewahrung der peruanischen Geschichte beteiligt ist. Ihr filmisches Werk ist von besonderer Wichtigkeit für die Aufklärung über die Folgen des bewaffneten Konfliktes in den 1980ern und 90ern. Dieser Konflikt sorgte in den Jahren zwischen 1980 und 2000 für Bürgerkrieg im Land, der laut des Informe final der Comisión de la Verdad y Reconciliación (CVR) 69280 Menschen das Leben kostete. Außerdem informiert der Film über die aktuellen sozialen Verhältnisse in Peru. Die Figur der QuechuaFrau oder indígena, zu deutsch Indiofrau, übernimmt dabei das Zentrum ihres Werkes. Sowohl ihr erster Film Madeinusa (2006) als auch die zweite Produktion La teta asustada (2009) wörtlich übersetzt bedeutet der Titel ,die erschrockene Brust“" - handeln von jungen Frauen aus den Anden, die sich ihren Platz in der Gesellschaft erkämpfen müssen, um dadurch ihr Überleben zu sichern. Beide Protagonistinnen werden von der zuvor unbekannten Schauspielerin Magaly Solier, Angehörige der Quechua, verkörpert. Besonders die Rolle der 
Fausta in La teta asustada ist ihr wie auf den Leib geschnitten. Dieser zweite Film wird Gegenstand der Analyse in dieser Arbeit sein. La teta asustada ist ein Drama, das sich an wahren Begebenheiten orientiert und fiktive Elemente hinzufügt. In La teta asustada wird das Schicksal von Fausta, einer jungen Frau, dargestellt, die in Zeiten des bewaffneten Konfliktes in Peru geboren wurde.

In diesem Film wird der Befreiungsprozess erzählt, den Fausta nach dem Tod ihrer Mutter bis $\mathrm{zu}$ deren Beerdigung durchlebt. Es ist ein Befreiungsprozess von traumatischen Erinnerungen, die nicht einmal ihre eigenen sind, sondern diejenigen ihrer Mutter. Diese musste in Zeiten des bewaffneten Konfliktes physische und psychische Qualen erleiden. Soldaten des peruanischen Militärs ermordeten ihren Ehemann und vergewaltigten sie während ihrer Schwangerschaft. Die traumatischen Erinnerungen übertrug sie, laut der allgemeinen Deutung der QuechuaFrauen, im Mutterleib und später mit der Muttermilch an ihre Tochter. Der Titel des Films beschreibt dieses intergenerationelle Phänomen, das laut Kimberley Theidon, auf deren Studie der Film basiert, von Quechua-Frauen und ihren Kindern im Departement Ayacucho nicht selten beobachtet wurde:

[L]as mujeres cuentan cómo los llakis (penas o males en quechua) se acumulan en sus cuerpos a causa de la violencia recibida, para luego ser transmitidos a sus hijos en el vientre materno y mediante la lactancia. Este mal es denominado 'la teta asustada' (Chauca et al. 2010: 46).

Um diesen Befreiungsprozess zu zeigen, hat Claudia Llosa den Minimalismus als Ausdrucksmittel gewählt. Sie verknüpft demnach die thematische Befreiung Faustas, d.h. die Loslösung von sozialen Ängsten und Schmerzen, die durch traumatische Erfahrungen der Mutter an sie übertragen wurden, mit der Reduzierung der Gestaltungsmittel. Die Entwicklung der Protagonistin ist ein schwieriger Prozess, der zeigt, wie groß die Resilienz oder menschliche Kapazität ist, traumatische Ereignisse ohne dauerhaft anhaltende Beeinträchtigung überstehen zu können. Der Film verdeutlicht auf drastische und dramatische Art und Weise, die Fähigkeit des Menschen, psychische und physische Schmerzen zu ertragen.

Ziel dieser Arbeit ist es nun zu untersuchen, inwiefern sich der minimalistische Einsatz von filmischen Elementen in der inneren Befreiung der Protagonistin Fausta von ihrer Vergangenheit, ihren Ängsten und ihres Traumas wiederspiegelt.

Als Grundlage werden im zweiten Kapitel zunächst die Filmdramaturgie und die Traumaforschung beschrieben und anschließend zueinander in Beziehung gesetzt. Beide sind für die Untersuchung des peruanischen Films La teta asustada relevant, da dieser über ein 
intergenerationelles Trauma aufklärt, das bis 2004 nicht an die Öffentlichkeit getragen wurde und dies auf eine einzigartige, künstlerische Art und Weise verarbeitet.

Kapitel drei wirft Licht auf die Geschichte Perus zur Zeit des bewaffneten Konfliktes zwischen 1980 und 2000. Es arbeitet die Hauptakteure und Folgen, die aus dem bewaffneten Konflikt resultieren, heraus. Zudem widmet sich das dritte Kapitel der Studie von Kimberley Theidon aus dem Jahr 2004 und diskutiert inwieweit diese als Vorlage oder Inspiration für Claudia Llosas Kunstwerk gesehen werden kann.

In der Analyse soll dann herausgefunden werden, inwieweit der Minimalismus eingesetzt wird, das heißt welche Gestaltungsmittel minimalisiert werden, wie sich dieser Minimalismus auch bei der Wahl der Figuren zeigt und welche Wirkung all dies auf den Zuschauer hat. Zudem soll hier der Frage nachgegangen werden, ob die Anwendung der minimalistischen Gestaltungsformen dem Zweck dient, dem Film eine noch authentischere und realistischere Form zu verleihen.

Die daran abschließende Schlussbetrachtung arbeitet die Zusammenhänge der einzelnen Kapitel heraus und bilanziert die bis dahin gewonnen Erkenntnisse. Der beigefügte Anhang enthält das Sequenzprotokoll, das für die Analyse angefertigt wurde, ebenso wie Screenshots der wichtigsten Sequenzen im Film zur bildlichen Veranschaulichung. 


\section{Theoretischer Hintergrund}

Die folgende Einführung in die Filmdramaturgie und die Traumaforschung dient dem besseren Verständnis der Analyse des Films. Beide wissenschaftlichen Bereiche enthalten Erkenntnisse, die für die Analyse des Films von Relevanz sind. Die Filmdramaturgie ist eine analytische Kategorie, deren Ziel die Beschreibung der Handlungsstruktur von Filmen ist. Wogegen in der Traumaforschung unter anderem die psychosoziale Traumaweitergabe diskutiert wird, was den Hauptkonflikt in dem hier zu analysierenden Film ausmacht.

\subsection{Filmdramaturgie}

Dramaturgie ist eine als eine Teildisziplin der Ästhetik tradierte praxisbezogene wie praxisbasierte Wissenschaft, die sich dem Geheimnis des Erzählens widmet und gleichermaßen analysiert sowie darstellt, was ein das Publikum unterhaltendes wie anregendes narrativ-performatives Werk ausmacht (Stutterheim/ Kaiser 2011: 15).

Die Filmdramaturgie befasst sich mit der Struktur und dem Ablauf einer Geschichte im Medium Film, das seit einigen Jahrzehnten zu einem der wichtigsten und einflussreichsten Medien des Geschichtenerzählens geworden ist. Im Gegensatz zu anderen Medien, wie beispielsweise Romane, sprechen Filme in der Regel mehrere Sinnenebenen gleichzeitig an. „Normalerweise besteht ein Roman aus einem einzigen verbalen Datenstrom“ (Hentschel/ Moormann 2018: 134), beim Film jedoch gibt es mehrere Informationsquellen: die Bild- und die Tonspur. Dabei kann die Tonspur noch aufgespalten werden, denn „Dialog, Geräusche und Musik werden oft simultan eingesetzt" (ebd.).

Erste grundlegende Aufgabe der Dramaturgie ist die Strukturierung der Ereignisabläufe, das heißt der Handlungen und Begebenheiten in der [...] Story zu dem Zweck, beim Zuschauer ein Interesse auf den Ausgang und das Ergebnis der Handlungen zu erregen (Rabenalt 2011: $34)$.

Dramaturgie beschäftigt sich mit den folgenden Fragen: Was soll erzählt werden? Was ist das Thema? Welche ästhetische Erfahrung oder Emotion soll beim Zuschauer oder bei der Zuschauerin ausgelöst werden? Und mit welchen Mitteln kann dies erreicht werden? Die Aufgabe eines Dramaturgen/ einer Dramaturgin ist es, innovative Inhalte zu finden und in jedem Moment des Films die gewünschte Wirkung zu erzielen, sowie die Kunst zu beherrschen, einen Spannungsbogen zu gestalten (vgl. Rabenalt 2011).

Grob kann unterschieden werden zwischen einer geschlossenen und einer offenen Dramaturgie. Die geschlossene Dramaturgie geht von einer 3-Akt-Struktur mit Anfang, 
Mitte und Ende aus. Mit einer geschlossenen Form werden grundlegende Bedürfnisse des Menschen befriedigt, wie beispielsweise das Verlangen nach Harmonie, Vollständigkeit, Konfliktlösung und Abgeschlossenheit.

Eine offene Dramaturgie stellt dagegen in der Regel eine größere Herausforderung für die Zuschauer dar. Sie lässt mehr Raum für Interpretation und erfordert eine aktive Beteiligung und mehr Aufmerksamkeit (vgl. Klotz 2012).

Mittlerweile existieren eine Vielzahl an Mitteln und Stilen in der Filmerzählung. Eines dieser Darstellungsmöglichkeiten ist die Verwendung des Prinzips weniger ist mehr oder less is more, von welchem auch Claudia Llosa in La teta asustada Gebrauch macht, um Inhalte zu vermitteln und die gewünschte Wirkung bei den Zuschauern zu erzielen. Die Analyse der Bauformen erfolgt im Kapitel 4.4 und 4.5.

Wenn also less zugleich more ist, so zielt das less eher auf formale Darstellungen, während das more die Wirkung auf den Zuschauer meint. Durch die Reduktion der üblichen Gestaltungsmittel, die die Erwartungen des Publikums prägen, auf ein Minimum, soll der Rezipient aktiviert und die ästhetische Wirkung auf ihn intensiviert werden. In diesem Sinne ist ästhetischer Minimalismus immer auch intendierte Maximalisierung der möglichen ästhetischen Erfahrung, im Film wie in anderen Künsten (Grob et al. 2009: 12).

Warren Motte beschreibt diese Vorgehensweise auch als lessening: „Through a reduction of means, minimalists hope to achieve an amplification of effect“" (Motte 1999: 4).

Das Kino des Minimalismus ist jedoch weder ein Genre, noch ein einheitlicher Stil. „Es ist eine besondere Ausdrucksweise, ein besonderes Gestaltungsprinzip, das sich [je nach Autor oder Autorin] in ganz unterschiedlichen Stilen äußert" (Grob et al. 2009: 12, Anm. der Verfasserin). Die Filmemacher, die minimalistische Prinzipien anwenden zielen darauf ab, durch stark reduzierte künstlerische und technische Mittel, „das Alltäglichste intensiv sichtbar, ästhetisch erfahrbar werden zu lassen“ und „das Allereinfachste in seiner bloßen Existenz zu würdigen“ (Grob et al. 2009: 10). Die Rede ist von einem „Kino des Knappen, Konzentrierten, Sparsamen, Elliptischen, [...] ein Kino des ent-dramatisierten, entpsychologisierten Handelns und Geschehens“ (Grob et al. 2009: 8). Dies bedeutet jedoch nicht, dass alle Gestaltungsmittel auf ihr Minimum verringert werden müssen.

Auf der narrativen Ebene, manifestiert sich der Minimalismus durch folgende Elemente: Echtzeitdarstellungen, elliptische Handlungsfolgen oder Aussparungen, lange Erzähldauer in einzelnen Sequenzen, Schilderung von Alltagsleben, flache Spannungskurven ohne Finalität, Fehlen oder Schwächung von Höhe- und Wendepunkten, schwache Konfliktentfaltung (vgl. Grob et al. 2009). 
Durch die Vermeidung von überladenen, belebten, symbolischen oder exotischen Räumen kann der Minimalismus auf der diegetischen Ebene gezeigt werden. Andere Möglichkeiten sind die Reduzierung der Anzahl von Figuren und Nebendarstellern, oder auch eine eingeschränkte Ausstattung mit wenig Dekor, Kostümvariationen und die Nutzung von realen Schauplätzen (vgl. ebd.).

Auf der visuellen Ebene bedeutet Minimalismus z.B. kein Übermaß an Reizen, keine Fülle von Farben oder Kontrasten (Monochromie), oder eine reduzierte Belichtung, Schärfe und Tiefenwirkung. Ebenso die Wiederholung einer bevorzugten, eventuell distanzierten Einstellungsgröße (Totale, Halbtotale, Halbnahe), die Kamera ist statisch, bewegt sich geruhsam und gleichförmig und verfolgt hauptsächlich die Bewegungen der Figuren (vgl. ebd.). Minimalismus auf der auditiven Ebene wird unter anderem durch lakonische Dialoge (kurz, einfach, ohne Bedeutung), Einzeiler und stumme Interaktion veranschaulicht. Die Atmosphäre wird mit sparsamen akustischen Modulierungen oder einzelnen musikalischen Akzenten geschaffen. In manchen Sequenzen fehlt es gänzlich an Geräuschen oder Musik (vgl. ebd.).

Auch Schauspiel und Inszenierung können minimal gehalten werden: Die Darsteller nutzen keine großen, ikonischen Gesten, sondern inszenieren ihr Schauspiel anhand von mimischer und gestischer Bewegungslosigkeit. Einige Filmemacher greifen daher eher auf Laien als auf professionelle Darsteller zurück, die zum Teil mehr Natürlichkeit ausstrahlen (vgl. ebd.). Minimalismus, in Form von ästhetischer und erzählerischer Ökonomie, kann als eine extreme Version des Realismus gesehen werden. Hier wird der Begriff Realismus als Stilmerkmal, im Sinne von Aktualität in Bezug auf das Zeitgeschehen, Exaktheit der zeitlichen und räumlichen Details, außerdem Authentizität bezüglich der politischen, sozialen, wirtschaftlichen und ideologischen Ereignisse, verwendet. Unterschiedliche Erscheinungsformen des Stilmerkmals teilen die Bemühung gegebene Tatsachen wahrheitsgetreu zu strukturieren und die ,sinnlich erfahrbaren Vorgänge mit den ihnen gemessenen einfachen [...] Mitteln im Gegensatz zu idealisierender Verklärung“ (Wilpert 2001: 662) zu gestalten.

\subsection{Traumaforschung}

In der Traumaforschung wird untersucht, unter welchen Umständen und auf welche Weise sich ein überwältigendes psychisches Ereignis auf das Gedächtnis und die Erinnerung 
auswirkt (Seidler et al. 2015: 15).

Die intergenerationelle Weitergabe von Traumatisierung wurde bisher hauptsächlich in Bezug auf die jüdischen Überlebenden des Holocaust untersucht, da hier eine Vielzahl an Forschungsergebnissen existiert.

„In diesem Kontext [wurde] ein integratives Modell transgenerationaler Übertragung“ (Seidler et al. 2015: 93) entwickelt. In diesem von Kellermann (2001) erstellten Modell werden folgende typische Befunde der intergenerationellen Traumatransmission aufgelistet:

Beeinträchtigter Selbstwert mit persistierenden Identitätsproblemen; Überidentifikation mit dem 'Opferstatus' der Eltern; hohe Leistungsorientierung, um den Verlust der Eltern zu kompensieren; Auftrag, Ersatz für verlorene Angehörige zu sein; Katastrophisierende Erwartungen; sorgenvolle Beschäftigung mit dem Thema 'Tod'; Belastung durch holocaustassoziierte Reize; Vernichtungsängste und Alpträume mit Verfolgung; Missstimmungen im Zusammenhang mit Gefühlen von Verlust und Trauer; ungelöste Konflikte im Zusammenhang mit Ärger und Schuld; übermäßige familiäre Bindung und Abhängigkeit oder übermäßige Unabhängigkeit und Schwierigkeiten, enge Bindungen einzugehen bzw. mit interpersonellen Konflikten umzugehen (Seidler et al. 2015: 94).

Es wird beobachtet, dass zwischen den Überlebenden des Holocaust und ihren Nachkommen zeitliche Grenzen zwischen dem Vergangenen und dem Gegenwärtigen nicht eingehalten werden.

Infolgedessen würden [...] elterliche Gefühle der Trauer und des Schmerzes sowie der unvergänglichen lebensbedrohlichen äußeren Realität auf das Kind übertragen, das dann die Gefühle nach außen trage, als wären diese seine eigenen. [Dies] erfolge dann, wenn die Eltern intensive Trauer und (selbstzerstörerische) Aggression wegen deren verheerender Natur psychisch nicht bewältigen und auch mit erwachsenen Partnern nicht teilen könnten (Seidler et al. 2015: 95, Anm. der Verfasserin).

Die traumatischen Erfahrungen der Eltern leben in ihren (Enkel-)Kindern wie ein Fremdkörper oder Parasit weiter und rauben ihnen die Möglichkeit und Energie, eigenen Bedürfnissen und Gefühlen gerecht zu werden und die eigene Identität zu entfalten (vgl. Seidler et al. 2015).

Die Kernidentität der Kinder wird überflutet und beeinflusst von verletzten Selbst und internalisierten Objektbildern nebst dazugehörigen Affekten, die den Eltern und Großeltern gehören (Lampartner et al. 2010: 13).

Zusätzlich zu den zeitlichen Grenzen werden auch Generationsgrenzen durchlässiger, ,[d]a die Kinder in zwei (biographischen) Wirklichkeiten lebten“ (Seidler et al. 2015: 96). Resultate dieser von den Eltern aufgezwungenen Erfahrungen und Emotionen sind, wie bereits oben erwähnt, große Identitätsprobleme, hohe Leistungsanforderung und auf lange Sicht schwere Depressionen (vgl. Seidler et al. 2015). 
Des Weiteren wird in Kellermanns Modell zwischen vier Weitergabeprozessen differenziert. Traumatische Erfahrungen können durch ,interpersonelle Beziehungen, Sozialisation, Kommunikation und genetische Disposition“ (ebd.) übermittelt werden. Unter genetischer Disposition notiert Kellermann eine „erbliche Vulnerabilität für PTBS“ (Posttraumatisches Belastungssyndrom) als zentralen Weitergabefaktor (Seidler et al. 2015: 94). An diese erbliche Vulnerabilität knüpft das Konzept der „fetalen Programmierung“ (Seidler et al. 2015: 101) an. Die wichtigsten Forschungsergebnisse in diesem Zusammenhang kommen von Rachel Yehuda in Zusammenarbeit mit ihrer Arbeitsgruppe. Die von ihr untersuchte Gruppe bestand aus schwangeren Frauen, die von der 9/11 Katastrophe betroffen waren. Sie konnte mehrfach in Studien zeigen, dass traumatischer Stress und auch Unterernährung in der Schwangerschaft zu einem erhöhten Glucocorticoidspiegel der Mutter führen können, was wiederum eine fetale Programmierung auslösen kann. Diese fetale Programmierung kann sich später in Form von physischer oder auch psychischer Beeinträchtigung zeigen (vgl. Yehuda et al. 2007).

Ein bedeutsames Element, das bisher noch nicht erwähnt wurde, jedoch relevant ist, um das Phänomen der psychosozialen Traumatransmission zu verstehen, ist das Schweigen, das Opfer traumatischer Erfahrungen bewahren. Das Verschweigen und die Negation der erfahrenen psychischen und physischen Gewalt verhindern die mentale Verarbeitung letzterer und verstärken den Effekt der eigentlichen gewalttätigen Erlebnisse. Kurz gesagt, das Schweigen darüber verhindert die Autoreparation und Wiederherstellung der Opfer (vgl. Iosa et al. 2013).

\subsection{Zusammenhang zwischen Filmdramaturgie und Traumaforschung}

Sowohl die Filmdramaturgie als auch die Traumaforschung gilt es nun hier in Verbindung zu setzen, da beide relevant für die Analyse des Films La teta asustada sind. Die Rolle des Films in der Vermittlung von Traumata darf nicht unterschätzt werden. Der Film kann eine künstlerische Form der Gesellschaftskritik sein, die wichtige Aufgabe der Aufklärung übernehmen und/ oder als Schuldbekenntnis gesehen werden. Gleichzeitig kann er zur Traumatherapie und Genesung beitragen. Der Prozess der Traumatisierung und dessen Überwindung können im Film durch Bilder, Ton, Geographie und Geschichte veranschaulicht werden (vgl. Mhando/ Tomaselli 2009). Kürzlich wurde der Begriff Cinema 
of Difficult Dialogues zur Benennung von Filmen vorgeschlagen, die bedeutende, soziale und die verschiedenen Volksgruppen betreffende Themen behandeln (vgl. ebd.). Im Gegensatz zu den Fachliteraturen der Psychologie, Soziologie, Geschichte und Ethnologie kann das Kino ein wesentlich größeres und breiter gefächertes Publikum erreichen. Des Weiteren ist zu beobachten, dass Audiovisuelles intensiver wahrgenommen wird als Geschriebenes oder mündlich Tradiertes. Filme besitzen daher reichlich Potenzial, um auf Menschen und deren Gedanken Einfluss zu nehmen. Deshalb können sie auch zu den oben genannten Zwecken effektiv genutzt werden.

Film and video generate interpretations in the minds of viewers, interpretation that can create a phenomenological sense of "being there," or "having been there." These are experiences of encountering the "real" (Mhando/ Tomaselli 2009: 31).

Das Gefühl der Anwesenheit, das der Zuschauer empfindet, verstärkt den Effekt, den der Film auf ihn haben soll.

In dem hier zu analysierenden Film La teta asustada dienen bekannte Phasen der Traumaverarbeitung zur Strukturierung des Films. Zu Beginn die Schockphase, danach folgt die Verarbeitungsphase und zuletzt die Entlastungsphase. La teta asustada erzählt in einem Handlungsstrang die Geschichte der persönlichen Entwicklung Faustas und der Verarbeitung ihres Traumas. Gleichzeitig ist es eine Konfliktgeschichte und die Geschichte einer Freundschaft beziehungsweise einer entstehenden Liebe, je nachdem auf welchen Aspekt des Films der Fokus gelegt wird. Der Film steigt direkt, ohne Umschweife, in die Haupthandlung ein. Es gibt keine Nebenhandlung oder Verschachtelung von Handlungssträngen, jede Szene des Films ist Teil der Haupthandlung. Der Film verläuft zeitlich linear. Große Zeitsprünge, Rückblenden oder Vorausdeutungen gibt es nicht. In 90 Minuten Filmzeit wird ein Zeitraum von ungefähr einem Monat dargestellt. Zeitraffungen finden zwischen den Sequenzen statt, das bedeutet, die dargestellten Ereignisse, geschehen in Echtzeit und zwischen den Sequenzen wird die Zeit sozusagen vorgespult. Echtzeitdarstellungen sind, wie bereits oben gesagt, ein Element des Minimalismus im Film.

Obwohl La teta asustada die individuelle Geschichte von Fausta schildert, ist es wichtig, diese als Teil innerhalb der peruanischen Landesgeschichte zu sehen und sie auf eine größere Zahl der peruanischen Bevölkerung auszubreiten. Das bedeutet, die traumatischen Erfahrungen, die Fausta beeinflussen, können als kollektives Gedächtnis gesehen werden. Sie leidet an einem „historical trauma“ (Espinoza 2010: 3). Das bedeutet, dass traumatische 
Erlebnisse bereits vor langer Zeit geschehen sind, diese aber immer noch ins Gedächtnis eingebrannt sind. Ein historisches Trauma beeinflusst die Gesundheit, das Leben und soziale Beziehungen über Zeit und Raum hinweg (vgl. Espinoza 2010). Gefühlszustände und Zustandsveränderungen werden in diesem Film durch Symbole sowie räumliche Metaphern und weniger durch Dialoge ausgedrückt. Die Minimalisierung der Gestaltungsformen verfolgt das Ziel, die innere Leere in Fausta und gleichzeitig ihren Befreiungsprozess zu visualisieren. Damit wird sich im Kapitel vier im Detail auseinandergesetzt.

\section{Intergenerationelle Traumatisierung in Peru nach Theidon}

La teta asustada ist ein Drama, das sich an wahren Begebenheiten orientiert. Der Film rekonstruiert die Gegenwartsgeschichte der heutigen Generationen in Peru. Er verfolgt die Intention, Außenstehende aufzuklären und der Gesellschaft $\mathrm{zu}$ ermöglichen, traumatische Ereignisse der nicht weit zurückliegenden Vergangenheit (1980-2000) zu verarbeiten.

Der Film basiert auf einer im Jahr 2004 veröffentlichten medizinisch-anthropologischen Studie von Kimberly Theidon mit dem Titel Entre prójimos: el conflicto armado interno y la política de la reconciliación en el Perú. In diesem Buch integriert Theidon Interviews, die sie mit Quechua-Muttersprachlerinnen aus der Region Ayacucho geführt hatte. Die Frauen erzählen von Vergewaltigungen und Missbräuchen, die ihnen sowohl von den Mitgliedern der Terrororganisation Sendero Luminoso, als auch vom peruanischen Militär angetan wurden (vgl. Chauca et al. 2010).

Um den Film, den in Kapitel vier analysiert wird, in seiner komplexen Gesamtheit wahrzunehmen und $\mathrm{zu}$ verstehen, ist es wichtig, auch den historischen Kontext sowie Theidons Studie miteinzubeziehen. Vor allem in Bezug auf diejenigen Elemente, die Claudia Llosa als Inspiration für ihren Film gedient haben. Das folgende Kapitel gibt einen Überblick über die wichtigsten historischen Ereignisse.

\subsection{Politische Gewalt in Ayacucho zwischen 1980 und 2000}

Der bewaffnete Konflikt in Peru ist ein interner Konflikt, der während der letzten zwei Jahrzehnte des vergangenen Jahrhunderts stattgefunden hat. An ihm waren hauptsächlich drei Parteien beteiligt, die unterschiedliche Ziele verfolgten. Die peruanische 
Regierung strebte die Realisierung und Festsetzung einer parlamentarischen Demokratie an, während die Sendero Luminoso diese lediglich wie eine Weiterführung der Militärdiktatur betrachtete. Die Sendero Luminoso, auch bekannt als Partido Comunista del Perú, ist eine maoistische Guerrilla Gruppierung, die zu Beginn zum größten Teil aus Studierenden der Universität von Ayacucho bestand. Sie kämpften für die Verbesserung der Lebensumstände im Departement Ayacucho, mit der Vision, das System zu demaskieren und im bewaffneten Kampf zu besiegen. Die dritte involvierte Partei ist die MRTA, Movimiento Revolucionario Túpac Amaru, eine linke Untergrundbewegung, die sich hauptsächlich aus der indigenen Bevölkerung zusammensetzte. Sie trat ein für ein freies und unabhängiges Vaterland, forderte die Nationalisierung ausländischer Firmen und Betriebe. Andere Ziele der MRTA waren die Partizipation der unterprivilegierten Bevölkerungsschichten, Begünstigungen für Lebensmittel und die Freilassung aller politischer Gefangener.

Die Gewalt der MRTA [hatte] ein wesentlich geringeres Ausmaß als die von Sendero Luminoso [...]. Man vermutet, daß [sic] die Revolutionäre Bewegung Tupac Amaru für 1\% der Opfer der politisch motivierten Gewalt zwischen 1984 und 1992 in Peru verantwortlich ist (Sierck 1997: 227, Anm. der Verfasserin).

Das Departement Ayacucho war dabei der Ausgangspunkt des bewaffneten Konfliktes, von dem aus die Mitglieder der Sendero Luminoso ihr ideologisches Vorhaben, die radikale Transformation der peruanischen Gesellschaft, dirigierten. Ebendort ereignete sich auch die erste von vielen bewaffneten Aktionen der Terrororganisation (vgl. Sánchez Villagómez 2015).

Estos hechos y muchos otros, establecieron una situación que produjo en el imaginario peruano, sobre todo urbano-limeño, la equivocada idea que los pobladores ayacuchanos eran simplemente 'agentes del mal'. Ayacucho quedó estigmatizado. Durante las décadas de 1980 y 1990 autorreconocerse como ayacuchano era un acto peligroso, puesto que se traducía, muy a menudo, como una declaración de parte que develaba su condición de terruco, término de uso popular que reemplazó a otro: terrorista (Sánchez Villagómez 2015: 9).

Die kommunistische Partei Sendero Luminoso entstand im Jahr 1970 als Ergebnis interner Kämpfe innerhalb der peruanischen kommunistischen Partei Bandera Roja. Die Führung übernahm Abimael Guzmán, der Ex-Nationalsekretär von Bandera Roja. Die Mehrheit der Mitglieder wurden an der Universidad Nacional de San Cristóbal de Huamanga rekrutiert. Unter ihnen Dozenten, Mitarbeiter und Studierende, welche zum größten Teil selbst aus den Provinzen Huanta, Huamanga, La Mar, Cangalla, Vilcashuamán und Víctor Fajardo stammten. Ganze 35,5\% der Terroristen, die zwischen 1983 und 1986 verurteilt und in Gewahrsam genommen wurden, haben eine universitäre Ausbildung genossen, „was 
überraschend hoch ist, wenn man berücksichtigt, daß [sic] nur 7,7\% der arbeitsfähigen Bevölkerung dieses Ausbildungsniveau hat" (zit. nach Chavez de Paz 1989: 43, Anm. der Verfasserin). $\mathrm{Zu}$ Beginn wurde die Terrororganisation stark unterschätzt und als vergleichsweise ungefährlich eingestuft.

Durante el periodo inicial (1980-1982), las acciones de SL se concentraron en el trabajo político antes que en el militar. Sus miembros implementaron tareas de propaganda, de organización de comités de apoyo y de organización de la producción y la distribución en algunas bases. No impusieron aún las ejecuciones de campesinos o de habitantes de los sectores urbanos populares, denunciados, muy a menudo de manera arbitraria, como 'soplones' o 'traidores a la revolución' (Theidon 2004: 28).

In ruralen Gebieten gewann Sendero Luminoso zunehmend an Macht, unter anderem, weil sie eine Lücke füllten, die der Staat hinterlassen hatte (vgl. Theidon 2004). Die zahlreichen Menschenrechtsverstöße der zur Polizei und Armee gehörenden fuerzas del orden verstärkten das Wachstum von Sendero Luminoso. Große Teile der Bevölkerung schlossen sich der Guerrilla Gruppierung an, um an der Polizei Rache zu üben. Diese Rachegelüste machte sich die Gruppierung zu Nutze, um Gleichgesinnte zu rekrutieren (vgl. ebd.). Um sich auch zukünftige Anhänger zu sichern, zwangen die Senderisten die Lehrer und Lehrerinnen in den Schulen der Umgebung ,revolutionäre Lieder“ zu singen und „den Marxismus ein[zuführen]“ (Sierck 1997: 228, Anm. der Verfasserin).

Wie bereits erwähnt, ist Sendero Luminoso für die Mehrheit der Opfer und Anschläge verantwortlich. Die folgende Tabelle fasst die Anzahl der von Sendero Luminoso ausgeübten Attentate zwischen 1980 und 1991 zusammen:

\section{Tabelle 1: Attentate der Terrororganisation Sendero Luminoso}

\begin{tabular}{|c|c|c|}
\hline Jahr & Anzahl der Attentate & \multirow{13}{*}{$\begin{array}{l}\text { Zahlen für 1980-1988: DESCO 1989: 25, } \\
\text { Zahlen für 1989-1990: Senado de la República 1991: } \\
\text { 10, } \\
\text { Zahl für 1991: IDL, in: Ideéle Nr. } 34 \text { (Feb. 1992): } 1 .\end{array}$} \\
\hline 1980 & 219 & \\
\hline 1981 & 715 & \\
\hline 1982 & 891 & \\
\hline 1983 & 1123 & \\
\hline 1984 & 1760 & \\
\hline 1985 & 2050 & \\
\hline 1986 & 2549 & \\
\hline 1987 & 2489 & \\
\hline 1988 & 2802 & \\
\hline 1989 & 2117 & \\
\hline 1990 & 2049 & \\
\hline 1991 & 2140 & \\
\hline
\end{tabular}

Es ist deutlich sichtbar, „dass sich die politische Kriminalität, die gegen den Staat gerichtet 
ist, [...] quantitativ geändert [hat]“" (Sierck 1997: 230, Anm. der Verfasserin). Im Jahr 1980, wurden 219 Anschläge gezählt, 1988 stieg die Zahl dagegen auf 2802 an. Zu Beginn richteten sich die Attentate insbesondere gegen Angehörige der Streitkräfte und wichtige Personen. Später traf die Gewalt auch die einfache Landbevölkerung:

Teilweise kam es zum Abbrennen ganzer Dörfer oder zu Massakern an Dorfbewohnern. Insbesondere Dörfer, in denen Selbstverteidigungseinrichtungen wie die 'Rondas' bestehen, wurden Opfer der Gewalt von Sendero Luminoso. Bei den 'Rondas' sind die freiwilligen Bürgerwehren im Norden des Landes von den 'Rondas' zu unterscheiden, die im südlichen Hochland von den Militärs im Rahmen der Aufstandsbekämpfung gegründet wurden. Die freiwilligen 'Rondas' gehen auf eine alte inkaische Tradition zurück. Die dienten den Dorfgemeinschaften zum Schutz vor Eindringlingen und waren ein System der informellen Sozialkontrolle (Sierck 1997: 231).

Wie auch die pájaros in Kolumbien, Auftragsmörder, die politische Feinde der konservativen Partei in den 70ern beseitigten, legten die Mitglieder von Sendero Luminoso ihre Opfer an öffentlichen Orten ab (vgl. ebd.). „Die Leichen wurden [darüber hinaus] oft mit Plakaten versehen. Auf diesen stand z.B. 'So sterben Verräter'" (ebd., Anm. der Verfasserin). Mögliche Gegner der Terrororganisation sollten durch diese Aktionen bedroht und eingeschüchtert werden.

\subsection{Situation nach dem bewaffneten Konflikt in Peru}

Im Jahr 2003 veröffentlicht die Comisión de la Verdad y Reconciliación (CVR) peruana ihren Informe final, ein Aufklärungsbericht mit erstaunlichen Ergebnissen zum bewaffneten internen Konflikt in Peru. Die Ergebnisse werden in diesem Teilkapitel genauer betrachtet.

Unter anderem enthält der Informe final, dass während der letzten beiden Jahrzehnte des 20. Jahrhunderts an die 69,280 Menschen ermordet wurden. Von diesen Opfern lebten $79 \%$ in ländlichen Gebieten und 75\% hatten als ihre Muttersprache Quechua oder eine andere indigene Sprache. Der Bericht macht die kommunistische Partei Sendero Luminoso für mehr als die Hälfte dieser Opfer verantwortlich (vgl. Theidon 2004).

Diesen Zahlen will Theidon in ihrer Studie nachgehen. Dafür interviewte sie 403 Personen, unter ihnen Frauen, Männer, Mädchen und Jungen (vgl. ebd.). Worauf Theidon vor allem aufmerksam machen möchte, ist die bürgerliche Beteiligung an den Morden. Sie versucht 
zu verstehen, was Menschen dazu treiben konnte, ihre eigenen Nachbarn oder Bekannten zu töten. In ihren Interviews taucht häufig der folgende Ausdruck als Beschreibung der Art und Weise der Morde auf: „matar entre prójimos“ (Theidon 2004: 21). Aufgabe dieser Aufklärungsstudie ist die Untersuchung der Veränderung der sozialen und politischen Strukturen, die noch heute die ethnische Diskriminierung, Ausgrenzung und Unruhe verstärken (vgl. Theidon 2004).

Die Besonderheit der guerras internas ist der Gegner oder Feind, denn es handelt sich um einen „'enemigo íntimo' - un vecino, una nuera, un padrino o la comunidad de enfrente“ (Theidon 2004: 22). Dieser Feind befindet sich in der direkten Umgebung. Jeder könnte es sein, was die Übergriffe noch unvorhersehbarer macht.

„El conflicto armado interno entre 1980 y 2000 fue el de mayor duración, intensidad y de más elevados costos humanos y económicos de toda la historia republicana peruana (Theidon 2004: 26).

Unter diesem internen Konflikt litt an erster Stelle das Departement Ayacucho, welches sich in unterschiedliche Gebiete aufteilt. Diese sind klar und deutlich zu identifizieren, da sie sich in Sachen Integration und Kommunikation stark unterscheiden. Ebenso unterscheiden sie sich durch die verschiedenen Eigentumsregelungen, die vor der Zeit der politischen Gewalt vorhanden waren. Diese Vielfältigkeit beeinflusste die Intensität der Gewalt während des internen bewaffneten Konfliktes in den 1980ern mit (vgl. Theidon 2004).

Das Gebiet im Norden des Departements, bestehend aus den Provinzen Huanta, Huamanga und La Mar, traf es am härtesten, gemessen an der Zahl der Anschläge und Opfer, sowie am Ausmaß der Zerstörung der Infrastruktur. Die Sendero Luminoso wurde aufgrund ihrer erbarmungslosen Gewalt stark kritisiert. Noch in der ersten Phase kam es schon zu Bombenattentaten in Lima. „Bis Ende 1982 starben 91 Zivilisten und 43 Angehörige der Streitkräfte durch Sendero Luminoso“ (zit. nach DESCO 1989: 37). Außerdem wurden zahlreiche Strommasten gesprengt, wodurch die Hauptstadt zum Teil Tage lang ohne Strom auskommen musste. Um Sendero Luminoso zu bekämpfen und die Schäden einzudämmen, wurden 1983 Einheiten der Armee und der Marine in das Gebiet geschickt (vgl. Sierck 1997).

Entraron las fuerzas armadas a comienzos del año 1983. Fue precisamente durante los años de 1983 y 1984 cuando la violencia alcanzó un nivel abrumador en las provincias de Huanta, La Mar, y Huamanga: en sólo esos años hubo la misma cantidad de muertos que en todos los años restantes del ciclo de violencia en la región" (zit. nach CVR 2003: IV, 93).

Als 1983 sieben Mitglieder von Sendero Luminoso von einer Gruppe Bauern aus Huaychao 
getöten wurden, lobte der damalige Präsident Fernando Belaunde „la 'heroicidad' de estos campesinos al defender al Estado peruano“" (Theidon 2004: 32). Andere Bauern der benachbarten Gemeinden in Huanta folgten diesem Beispiel, indem sie sich auf die Seite des Staates schlugen:

Las demás comunidades de las alturas de Huanta escuchaban, y [...] decidieron 'rescatar su imagen' por medio de una toma de posición en contra de los senderistas, forjando una alianza conflictiva pero estratégica con las fuerzas armadas. Parte de esta alianza consistió en formar sus rondas campesinas. En el proceso de organización contra Sendero, se decidieron a 'limpiar sus comunidades', erradicando a los simpatizantes (Theidon 2004: 32).

Das Gebiet im mittleren Süden des Departements Ayacucho wurde von der Anschlagreihe weniger heimgesucht als der Norden, trotzdem gab es schwerwiegende Folgen.

Fue en esta zona centro-sureña donde el PCP-SL [el Partido Comunista del Perú] logró un apoyo prolongado importante. Fueron los mismos hijos del lugar quienes empezaron a desarrollar un adoctrinamiento a través de su posición como 'educados'- profesores en una región donde la educación simbolizó 'el progreso' y la movilidad social (Theidon 2004: 35, Anm. der Verfasserin).

Die Dörfer der Gegend mussten wiederholt Anschläge des Ejército erdulden: „,T]eniendo como resultado dos de las masacres emblemáticas del conflicto armado interno: Lloqllepampa-Accomarca, el 14 de agosto de 1985, y Cayara, el 14 de mayo de 1988“ (ebd.). Die Auswirkungen dieser Gewalt auf das Leben und die körperliche sowie psychische Verfassung der ländlichen Bewohner war und ist immer noch gewaltig. Häufig wird diese Erbarmungslosigkeit als sinnlos beschrieben (vgl. ebd.).

[P]uede ser que la violencia horrenda destruya los significados aceptados y los vocabularios compartidos, y asalte los órganos sensoriales. [...] La violencia efectúa un asalto tremendo sobre los sentidos y los significados. Muchos comuneros [...] han dicho que lloraron hasta perder la visión; [...] han asegurado que cuerpos que cargan tanta tristeza son cuerpos que duelen y que envejecen antes de tiempo. [...] Dentro del paradigma de la somatización, el cuerpo es el vehículo pasivo para la expresión del dolor psíquico y emocional (Theidon 2004: 48/49, Anm. der Verfasserin).

Auch nicht körperliche, traumatische Erfahrungen können durchaus zu schwerwiegenden Erkrankungen führen. Diese Verbindung zwischen dem seelischen und körperlichen Wohlbefinden fällt in den medizinischen Fachbereich der Psychosomatik. Die Somatisierung ordnet körperliche Beschwerden, die jedoch nicht auf konkrete organische Befunde oder bewiesene körperliche Fehlfunktionen zurückzuführen sind, trotzdem den körperlichen Erkrankungen zu, die eine medizinische Behandlung erfordern. Neben physischer Gewalt waren die Menschen auch enormen psychischen Belastungen ausgesetzt, wie z.B. Drohungen, Isolation, mangelnde Integration, ethnische Diskriminierung etc. In 
Interviews personifizieren die Landbewohnerinnen häufig ihre Trauer oder andere negative Gefühle:

Una señora en Accomarca [...] habló de su pena y cómo el estar sola le resultaba muy difícil porque 'Cuando estoy sola, mi tristeza me sigue y quiere agarrarme'. De modo similar, podemos pensar en el alcanzo, que agarra a una persona que pisa o se sienta donde no debe, provocando así a los gentiles, responsables del alcanzo. (Theidon 2004: 58).

Andere comuneros erzählen, dass sie aufgrund der erfahrenen Gewalt und ihrer tiefsitzenden Trauer bis zur Erblindung weinten. Diese körperliche Sprache ist der westlichen Medizin und ihrer klinischen Definition von Somatisierung unbekannt (vgl. Theidon 2004).

In diesem Zusammenhang stellt Theidon die Theorie des Embodiments nach dem Ansatz von Csordas (1994) vor. Die Theorie beschreibt eine Reaktion auf posttraumatische Situationen, die der Körper erfährt, sowie Fragen die daraus resultieren. Csordas zufolge erklärt sich jede körperliche Erfahrung in einem Dialog zwischen der Kultur und dem Selbst (vgl. Theidon 2004). Es existiert kein Selbst als alleiniges Bewusstsein, das sich unabhängig vom dazugehörenden Körper entwickelt. Das Selbst bildet eine Einheit mit dem Körper, sowie mit der Kultur. Somit werden posttraumatische Emotionen durch den Körper nach außen getragen und wahrnehmbar gemacht.

In Theidons Studie werden verschiedene Krankheitsbilder geschildert, die in der Region Ayacucho von den ländlichen Bewohnern beobachtet wurden. Die quechuasprachige Bevölkerung in Ayacucho glaubt an die Theorie, der zufolge Kinder ohne Seele geboren werden. Diese entwickelt sich erst innerhalb der ersten beiden Lebensjahre, was bedeutet, dass die Seele im Kindesalter nicht fest genug im Körper verankert ist. Das bedeutet, der Status des „runakuna“ (Theidon 2004: 60) oder der Status des Menschseins und die Identität eines Menschen konstruieren sich mit der Zeit. Außerdem definieren sich die Menschen durch ihre sozialen Beziehungen, durch ihren sozialen Kontext, durch die Liebe, die sie in anderen Menschen wecken.

Da die Seele noch nicht ausreichend im Körper der Kleinkinder gefestigt ist, sind sie anfälliger für den susto, ein lang anhaltender Schockzustand, der durch negative Gefühle und Erinnerungen provoziert wird. Kinder, die unter der politischen Gewalt leiden mussten und das Krankheitsbild des susto bestätigen, mangelt es an der Fähigkeit Liebe in anderen Menschen zu wecken oder sie selbst zu empfinden (vgl. Theidon 2004).

Die „llakis“ (Theidon 2004: 64) formen ein Leiden, das sowohl durch politische Gewalt, als auch durch Armut ausgelöst werden kann und an schmerzvolle Erinnerungen geknüpft ist. Dieses Leiden kann den ganzen Körper einnehmen und die betroffene Person spürt 
überwältigende Schmerzen, sowie Atemnot und Ohnmachtsanfälle.

Weitere Krankheitsbilder sind „Iquyasqa“ (Theidon 2004: 72), ein Zustand der konstanten Schwäche und Energielosigkeit und „la teta asustada“ (Theidon 2004: 77), eine Traumatisierung, die von der Mutter an ihr im eigenen Leib heranwachsendes Kind und nach der Geburt über die Muttermilch weitergegeben wird. An dieser Stelle ist es wichtig, wiederzugeben, was in Accomarca, nach dem Massaker im Jahr 1985, beobachtet wurde.

Al tiempo de la matanza del 14 de agosto de 1985, varias mujeres estaban embarazadas. Algunas estaban a punto de dar a luz, mientras que otras darían a luz en las semanas que siguieron inmediatamente a la matanza. Actualmente hay siete jóvenes que nacieron por esas fechas, y todos tienen problemas físicos y/o mentales. Sufren de ceguera o sordera, o son sordomudos; dos tienen epilepsia. Lo que queda claro es que, en todos los casos, las madres de estos jóvenes fueron testigos presenciales de la matanza de Lloqllepampa. Vale la pena considerar la relación que los comuneros y comuneras construyen entre la violencia horrenda y el nacimiento de bebés "dañados." (Theidon 2004: 77).

Das nächste Unterkapitel wird nun einen Zusammenhang zwischen Theidons Studie und Llosas Film herstellen. Außerdem wird hervorgehoben, welche Elemente der Interviews in der Studie im Film La teta asustada verarbeitet wurden.

\subsection{Zusammenhang zwischen Studie und Film}

In diesem Teilkapitel soll der Frage nachgegangen werden, inwieweit die Studie von Theidon als Vorlage oder Inspiration für Claudia Llosas Film La teta asustada gedient hat. Es wird sich denjenigen Elementen gewidmet, die im Film verarbeitet wurden.

Aufgabe des Films ist es, die Geschichte aufzuarbeiten und dem Vergessen entgegenzuwirken, sowie an die Opfer und die Folgen des Konfliktes zu erinnern. Wie auch in Llosas erstem Film behandelt sie das Thema der sozialen Unterdrückung und der aufgrund ihres Geschlechts diskriminierten Frau.

Der historische Hintergrund wird im Film lediglich angedeutet. Dies geschieht durch die Antagonistin Aída, die in Kapitel vier näher analysiert wird. An dieser Stelle ist es jedoch wichtig zu erwähnen, dass die Geschichte Perus - der Bürgerkrieg und die soziale Ungleichheit zwischen indígenas und von Europäern abstammenden Mestizen - durch die Wanddekoration in Aídas Schlafzimmer angedeutet wird. Diese besteht aus Familienporträts, unter welchen das eines Offiziers in Uniform ganz besonders hervorsticht.

Das Bild ruft Ängste in Fausta hervor. Die Beschreibungen der von Theidon interviewten Frauen angesichts der Krankheit la teta asustada, stimmen mit Faustas Beschwerden überein. Sie scheint keine Seele zu haben. Anstelle einer Seele, sitzen Ängste und Schmerzen tief in 
ihrem Inneren. Sie leidet an Ohnmachtsanfällen und Nasenbluten.

Die angesprochene Theorie des Embodiments oder Somatisierung des Traumas wird mit der Maßnahme verdeutlicht, zu der Fausta greift, um sich vor Angriffen zu schützen. Fausta führt sich zur Abwehr eine Kartoffel in die Vagina. So verhindert sie sexuelle Übergriffe. Die Kartoffel steht symbolisch für das Trauma, das Fausta in sich trägt (vgl. Bernales Albites/ Gómez 2017).

Así, al insertarse la papa en la vagina para protegerse de cualquier futura violación como la de que fue objeto su madre, Fausta [...] mostraría cómo se usa el cuerpo para expresar las emociones del ser en estado postraumático dentro de una cultura determinada, en este caso la andina donde el tubérculo adquiere un gran significado simbólico" (Bernales Albites/ Gómez 2017: 95).

In Theidons Studie wird diese drastische Maßnahme nicht erwähnt, es ist demnach ein fiktives Element im Film. Anhand der Ablehnung angesichts des Traumas vonseiten des Arztes im Film, den Fausta nach einem Ohnmachtsanfall aufsucht, wird verdeutlicht, wie die Reaktion der westlichen Medizin auf die Krankheit namens la teta asustada war. Mit der bildlichen Beschreibung der unterschiedlichen Krankheitsbilder von Seiten der Betroffenen konnte nicht angemessen umgegangen werden (vgl. Theidon 2004). Es wurden andere Mittel und Wege gesucht, um mit diesen Krankheitsbildern und der Traumatisierung umzugehen. Unter anderem wurden Heiler aufgesucht, die den Körper der Betroffenen reinigen sollten. „En los procesos de curación que utilizan los campesinos, hay mucho énfasis en "la limpieza". Es decir, ya sea por "pasar" un huevo o un cuy o por ir a la iglesia evangélica para quitarse sus llakis (memorias penosas)" (Theidon 2004: 59).

Die Reinigung oder der Befreiungsprozess, den Fausta im Film durchlebt, wird nun in der Analyse im folgenden Kapitel beschrieben. Dabei soll die Betonung auf der Untersuchung der Gestaltungsmittel liegen. Diese wurden in reduzierter Form eingesetzt, um die besagte Befreiung des Traumas la teta asustada zu visualisieren. 


\section{Filmanalyse: La teta asustada (2009)}

Diese folgende Analyse widmet sich der Frage, inwiefern der minimalistische Filmstil genutzt wird, um die Konzentration auf die Handlung, die innere Befreiung der Protagonistin, zu lenken. Das heißt, es soll beobachtet werden, wie Faustas Befreiung von ihrer Traumatisierung anhand filmischer Gestaltungselemente veranschaulicht wird. Es gilt die These zu untersuchen, ob die Anwendung der minimalistischen Gestaltungsformendem Zweck dient, dem Film eine noch authentischere und realistischere Form zu verleihen.

\subsection{Methode}

Generell kann eine Filmanalyse viele Bereiche im Detail analysieren und dabei sehr umfangreich werden, denn ein Film besteht aus einer Vielzahl von Komponenten. Darum beschränkt sich diese Filmanalyse auf jene Aspekte, die dem Zuschauer Aufschluss über die Darstellung von Faustas Befreiung geben. Wichtig für die Analyse dieser Arbeit sind Gestaltungskomponenten, wie beispielsweise die Architektur des ruralen und urbanen Raums und die Mise-en-scène im Film. Letztere bezieht sich auf alles, was vor der Kamera auftaucht, genauso wie dessen Anordnung, die so den Raum bildet. Dies wird auch als das set design bezeichnet, die Bildinszenierung oder die Bildkomposition. Folglich und im Rahmen dessen, wird in der Analyse vor allem das Setting eine wichtige Rolle spielen. Dazu gehört zum Beispiel Kostüm und Make-up der Figuren, da dies die Charaktere des Films beschreibt und unterscheidet. Dieser Aspekt ist deshalb wichtig, da die Filmfiguren wesentlich zur Raumformung beitragen. Make-up und Frisuren können Anzeichen von Veränderung innerhalb der Charaktere darstellen. Ein weiteres wichtiges Element, welches es zu untersuchen gilt, ist die Dekoration der Räume. Und zwar deshalb, weil die Objekte, darunter Symbole, im Film ausschlaggebend für die Raumkonstruktion sind. Immer noch in der Mise-en-scène, jedoch eher in der virtuellen Inszenierung, wird dem Licht im Film Aufmerksamkeit geschenkt. Dieses schafft Aspekte des Raums in den Vorder- oder Hintergrund und kann auch Farben beeinflussen. Außerdem beeinflusst es das Ambiente des Raums (vgl. Barsam/ Monahan 2010), wobei von Frontallicht, Gegenlicht, Seitenlicht oder der Art von Beleuchtung gesprochen werden kann. Das Licht kreiert außerdem Plastizität, verändert den Raum, charakterisiert den Raum und zeigt, was man von ihm sehen soll. Somit kann hier auch von einer Architektur des Lichts gesprochen werden. Die nächsten Elemente, 
die im Bereich der Mise-en-scène untersucht werden sollen, sind der Fokus, der die Aufmerksamkeit auf bestimmte Aspekte des Raums lenkt und die Komposition, die Raum durch die Anordnung von Objekten und Personen schafft (vgl. Pramaggiore/ Wallis 2005). Des Weiteren werden die Figuren im Film analysiert. Der filmische Raum ist der Handlungsraum der Figuren, deren Charakter oder Handlungen mithilfe des Raums, in dem sie sich bewegen, gedeutet werden. Abgesehen von der Untersuchung der Mise-en-scène, wird die Filmanalyse auch die Einstellungsgrößen der Kamera und die Kameraperspektive in den Blick nehmen. Bestimmte Elemente des Raums können in den Einstellungen extreme close-up/ Detailaufnahme, close-up/ Großaufnahme, close shot/ Nahe Aufnahme, medium shot/ Mittelgroße Aufnahme, full shot/ Halbnahe Aufnahme, medium long shot/ Halbtotale Aufnahme, long shot/ Totale Aufnahme und extreme long shot/ Weite Aufnahme gezeigt werden. Der Fokus liegt dabei auf einem bestimmten Bildausschnitt, einem Charakteristikum einer Person oder einem Objekt, das im Bild zu sehen ist. All das ist entscheidend für die Wirkung der Bildaussage. Das ersichtlichste Untersuchungselement ist der Inhalt an sich, da die darin stattfindenden Handlungen Resultat des Raums sind und diesen gleichzeitig konstruieren und verändern. Des Weiteren wird der Ton in einer ebenfalls semiotischen Analyse untersucht, in Form des Bildtons, des Fremdtons, der Effekte, des OnTons (Quelle des Tons im Bild sichtbar), des Off-Tons (Quelle des Tons außerhalb des Bildes), der Filmmusik oder auch der Dialoge.

Diese oben genannten Elemente werden sowohl anhand von relevanten Sequenzen als auch der Handlungssegmente untersucht, die durch Ort und Zeit gekennzeichnet sind. Eine Sequenz versteht sich als Handlungseinheit, die meistens mehrere Einstellungen beinhaltet. Für die Analyse wird für den gesamten Film ein Sequenzprotokoll, das im Unterkapitel 7.1 angehängt wird, als „Gedächtnisstütze [oder] die Festlegung von positiven Daten“ (Becker/ Schöll 1983: 28, Anm. der Verfasserin) angefertigt. Dieses wird die Nummer der Sequenz, den Zeitpunkt innerhalb des Films, die Dauer der Sequenz, die Orte, die Handlung, die Figuren und deren Kostüme und Maske, Musik und Geräusche, Momente der Stille, die Einstellungsgröße, Kameraperspektive und Kamerabewegungen, Licht und Farbe, den Bildinhalt und die Komposition beinhalten.

Die Einheit des Ortes beziehungsweise der Ortswechsel dient in diesem Fall als Basiseinheit für die Einteilung der Sequenzen. Für die Anfertigung eines detaillierten Sequenzprotokolls bietet sich „die gezielte Fragmentierung der Wahrnehmung [an]: Die Bildanalyse wird befördert durch die Ausschaltung des Tons, die Musik- und Geräuschanalyse durch die 
Abdeckung oder Schwärzung des Monitors“ (Faulstich 2002: 113, Anm. der Verfasserin). So können einzelne Elemente klarer und eindeutiger wahrgenommen werden.

\subsection{Peruanische Filmkultur}

Im Gegensatz zu großen Filmindustrien wie Hollywood oder Bollywood, selbst im Vergleich zu anderen südamerikanischen Ländern, ist die peruanische Filmindustrie international weitestgehend unbekannt. 1899 wurden im Teatro Politeama de Lima die ersten bewegten Bilder mithilfe eines Stereokinematographen ausgestrahlt. Diese ersten Produktionen konzentrierten sich zunächst auf geographische Aufnahmen Perus, unter ihnen La Catedral de Lima, der Camino de La Oroya und die Provinz Chanchomayo. Sie stellten den Anfang einer Reihe von Dokumentaraufnahmen der peruanischen Landschaft und ihrer Bevölkerung dar. Vom Publikum geschätzt wurden exotische Zeremonien, Feste, Riten, Kolonisierungs- oder Evangelisierungsarbeiten, die die nationale Filmwelt bestimmten (vgl. Bedoya 2002).

Der erste peruanische Spielfilm Negocio al agua wurde schließlich 1913, noch ohne Ton, veröffentlicht. In den Jahren zwischen 1919 und 1930 dienten die Produktionen größtenteils zur Berichterstattung über die damaligen aktuellen politischen Ereignisse geknüpft an Augusto B. Leguía und seine autokratische Regierung. Zur selben Zeit wurden aber auch Fiktionfilme gedreht, wie beispielsweise 1922 Camino de la venganza, ein Drama des Fotografen und Malers Luis Ugarte. Regisseure wie Ugarte, Enrique Cornejo Villanueva und Enzo Longhi versuchten zu beweisen, dass ebenso in Peru ein Kino geschaffen werden könnte, das das internationale Publikum begeistern würde (vgl. ebd.).

Weitere Entwicklungen folgten im Jahr 1936 mit Buscando olvido und Sigifredo Salas in der Regie, als sich der Stummfilm zum Tonfilm entwickelte. Für die Weiterentwicklung des Tonfilms spielte ebenso die neu gegründete Firma Amauta Films, die allein zwischen 1937 und 1940 vierzehn Spielfilme, darunter Dramen und Komödien, produzierte. 1940 kam die nationale Produktion von Filmen aus mehreren Gründen plötzlich zum Stillstand. Zum einen der Mangel an Investoren verursacht durch den 2. Weltkrieg, zum anderen einige wirtschaftliche Zwischenfälle hervorgerufen durch die Zensur eines von Amauta Films produzierten Films namens Barco sin rumbo. Aber vor allem waren es die mexikanischen Produktionen, die das Publikum in ihren Bann zogen und immer mehr an Bedeutung für das 
spanischsprachige Kino gewannen (vgl. ebd.).

Einen neuen Versuch, erfolgreiches Kino in Peru zu kreieren, machte der Foto Cine-Club Cuzco, der 1955 gegründet wurde. Es waren die Cineasten aus Cuzco, die sich in ihren dokumentarischen Produktionen mit der Landbevölkerung der Anden, sowie den Lebensbedingungen der indígenas auseinandersetzten. Dadurch konnte ein Einblick in ihre Lebensweise aus kultureller Sicht gewährt werden. In dieser Rubrik stachen vor allem die Dokumentationen des Regisseurs Manuel Chambi hervor (vgl. ebd.).

In den 50er Jahren wurde lediglich ein Spielfilm in Lima ausgestrahlt, La muerte llega al segundo show von José Marie Roselló. Durch diese Produktion wurden der technische Rückschritt und der Mangel an professionellem Equipment sichtbar, die das Ergebnis jahrelanger Passivität der Filmproduktion in Peru waren (vgl. ebd.).

1958 konnte Peru sich für Fernsehsendungen begeistern und von diesem Zeitpunkt begann die Filmindustrie stetig an zu wachsen. Die Schauspieler, die durch telenovelas im Fernsehen bekannt wurden, übernahmen ebenfalls Rollen in den Kinoproduktionen. Durch Werbung auf internationaler Ebene wurde das peruanische Kino von nun an außerdem durch Zeitschriften wie Hablemos de cine unterstützt (vgl. ebd.).

Durch das im Jahr 1972 verabschiedete Ley de Promoción a la Industria Cinematográfica wurde zum einen festgelegt, dass die Filmvorführung der peruanischen Kinofilme obligatorisch würde und zum anderen, wie viele Anteile der Steuern den Produzenten zugutekommen sollten. Der Erfolg dieses Gesetzes war sofort sichtbar und führte zu einer Wiedergeburt des peruanischen Kinos. In den darauffolgenden 20 Jahren wurden in Peru derart viele Filme gedreht, wie noch nie zuvor. Einige Produktionen, die zu der Zeit entstanden, wie La ciudad de los perros von Lombardi oder La fuga del chacal von Augusto Tamayo San Román, konnten sogar mit den Blockbustern aus den USA konkurrieren (vgl. ebd.).

Unglücklicherweise konnten die Kinosäle aufgrund der Wirtschaftskrise und des Terrorismus nicht mehr ausreichend gefüllt werden, da die Eintrittskarten lediglich für die obere Mittelschicht bezahlbar waren. Daraufhin wurde 1994 ein neues Gesetz erlassen, das die Produktionen durch Wettbewerbe und Preise ankurbeln sollte. Leider ohne Erfolg (vgl. ebd.).

In der ersten Hälfte der 80er Jahre wurden die Themen Terrorismus, bewaffneter Konflikt und Informationen über die Kommunistische Partei Perus Sendero Luminoso im peruanischen Kino gemieden. Erst Ende der 80er Jahre fanden Filmemacher Interesse an 
diesen Themen. Regisseure, wie Francisco Lombardi und Marianne Eyde, produzierten Filme, die über den bewaffneten Konflikt informieren und Unbeteiligte aufklären sollten. Ersterer studierte das Verhalten und Motive der Soldaten, die in den Anden gekämpft hatten. Letztere konzentrierte sich auf eine Gemeinde in den Anden, die zwischen den Rebellen von Sendero Luminoso und den Soldaten der Regierung gefangen war (vgl. Barrow 2014). Aufgrund einiger Veränderungen der Regierungspolitik in den 90er Jahren, bezüglich der staatlichen Finanzierung des nationalen Kinos, wurde der Fokus auf Produktionen gesetzt, die die breite Bevölkerung in Peru befriedigen sollten, wie Komödien, historische Dramen und Melodramen. Dies bedeutete, dass die kinematographische Bearbeitung der politisch motivierten Gewalt in Peru wieder vermieden wurde (vgl. ebd.).

Erst Anfang der Jahrtausendwende wurden die Themen erneut aufgegriffen und auf verschiedenste Weise bearbeitet. Fabrizio Aguilar verfilmte 2003 in Paloma de Papel

the point of view of a young boy to explore the trauma suffered by an Andean village located at the epicentre of the violence, and was the third most popular film at Peru`s box office in the year of its release (Barrow 2014: 4).

Nach zehn Jahren war es der erste in Peru produzierte Film, der sich die Gewalt des Sendero Luminoso zum Thema machte und von einer staatlichen Förderung profitierte. Darauf zeigte Josué Méndez einige Monate später in Días de Santiago ,an intimate portrayal of a young 'veteran' of armed conflict as he tries to return to family and civilian life in Lima“" (ebd.). 2009 wurde dann der hier zu analysierende Film La teta asustada von der Jungregisseurin Claudia Llosa ausgestrahlt. Ein Film, der sich mit den Folgen des bewaffneten Konfliktes in Peru auseinandersetzt und dies aus der Sicht einer Frau aus der Folgegeneration. Fausta war somit nicht direkt von der Gewalt betroffen und weist trotzdem noch Symptome einer Posttraumatischen Belastungsstörung auf. Dies veranschaulicht die Schwere der politischen Vergehen an der indigenen Gesellschaft.

Gedreht wurde der Film in Spanien und Peru, produziert von Vela Producciones, Wanda Visión S.A. und Oberón Cinematográfica S.A. Die Montage wurde von Frank Gutiérrez übernommen, Selma Mutal war für die außerordentlich einzigartige Filmmusik verantwortlich. Die Besetzung stammt zum größten Teil aus Peru. Die Darstellerin Magaly Solier, die die Rolle der Fausta verkörpert, stammt tatsächlich aus dem vom bewaffneten Konflikt meist betroffenen Gebiet Huanta. Die einzige Ausnahme bildet Susi Sánchez, die spanische Schauspielerin, die die Rolle der Aída verkörpert. Der Film kann nicht nur aus diesem Grund als authentisch und wahrheitsgetreu gekennzeichnet werden. Die Anlehnung 
an Theidons Studie, die unveränderte Darstellung der gezeigten Orte und die Abbildung gesellschaftlicher Probleme unterstützen diese These.

Ein detailliertes Resümee des Films folgt im kommenden Unterkapitel. Mit La teta asustada gewann Claudia Llosa weltweite Anerkennung. Sie wurde belohnt mit namenhaften Auszeichnungen, unter ihnen der Goldene Bär der Berlinale. Außerdem wurde der Film 2010 für einen Oscar in der Kategorie „bester ausländischer Film“ nominiert.

\subsection{Synopsis von La teta asustada}

Dieses Unterkapitel wird eine Zusammenfassung des Films liefern. In La teta asustada wird das Schicksal von Fausta, einer jungen Frau, dargestellt, die in Zeiten des bewaffneten Konfliktes in Peru geboren wurde. Dieser Konflikt sorgte in den Jahren zwischen 1980 und 2000 für Bürgerkrieg im Land, der laut des Informe final der Comisión de la Verdad y Reconciliación (CVR) 69280 Menschen das Leben kostete. Das Epizentrum des Konfliktes war die Andenregion vor allem südlich der Hauptstadt Lima, eines der ärmsten Gebiete des Landes (vgl. Theidon 2004, zit. aus CVR 2003: conclusiones).

Aus besagtem Gebiet stammen Fausta und ihre Mutter Perpetua. Als Perpetua mit Fausta schwanger war, musste sie unvorstellbare Gräueltaten über sich ergehen lassen. Ihr Ehemann Josefo wurde vor ihren Augen ermordet, sie wurde brutal vergewaltigt und wurde gezwungen den abgetrennten Penis ihres Mannes zu essen. Fausta erlebte diese Gewalt im Bauch ihrer Mutter mit.

Der Film spielt zum größten Teil in Manchay, einem sich konstant vergrößernden Armenviertel am Rande Limas, wohin die Mutter mit Fausta nach der am eigenen Körper erfahrenen Gewalt, geflohen ist. Dort leben die beiden gemeinsam mit der Familie des Onkels Lúcido. Dieser organisiert erfolgreich Hochzeiten, denen viele Szenen im Film gewidmet sind.

Trotz des Umzugs kehren die beiden immer wieder, in Gedanken und in selbst kreierten Liedern auf Quechua, zurück zu ihren traumatischen Erfahrungen und können diese nicht verarbeiten. Fausta leidet an la teta asustada, eine Krankheit, welche sie daran hindert, ein normales Leben zu führen. La teta asustada beschreibt ein Trauma, welches Mütter über die Muttermilch an ihre Kinder weitergeben, laut der Interpretation der indigenen Gesellschaft. Die konstante Präsenz der Erinnerungen an die Brutalität, die Faustas Mutter erlebt hat, 
verschlimmert den psychischen und auch physischen Zustand der jungen Frau. Zu Lebzeiten verarbeitet die Mutter ihre Erlebnisse mithilfe ihres Gesangs und bezieht Fausta auf diese Weise mit ein. Fausta lebt daher in konstanter Angst vor gewaltsamen sexuellen Übergriffen. Aus panischer Angst vergewaltigt zu werden, führt sich Fausta zum Schutz vor sexuellen Übergriffen unter großen Schmerzen eine Kartoffel in die Vagina ein. Ebenso ist Fausta nicht in der Lage ohne Begleitung das Haus zu verlassen, denn jeder fremde Mann stellt eine potentielle Gefahr für sie dar. Auch körperlich leidet Fausta unter ihrem Trauma: In besonders stressigen und beängstigenden Situationen bekommt Fausta Nasenbluten und wird ohnmächtig.

Der Tod ihrer Mutter am Anfang des Filmes stellt Fausta vor eine große Herausforderung. Da die Beerdigung der Mutter in Manchay oder Lima keine Option für Fausta ist, benötigt sie eine erhebliche Summe an Geld, um die Mutter in ihrem Heimatort in der Provinz in Würde begraben zu können. Aus einfachen Verhältnissen stammend, verfügt Fausta nicht über die nötigen finanziellen Mittel. Ebenso wenig ihr Onkel, der selbst nur das Nötigste bezahlen kann und die kostspieligen Hochzeitswünsche seiner Tochter Máxima erfüllen muss.

Um die letzte Reise ihrer Mutter zurück in die Heimat zu verwirklichen, entscheidet sich Fausta dazu, den Leichnam einzubalsamieren, bis sie das Geld aufgetrieben hat. Sie nimmt eine Arbeit als Hausmädchen in Lima an und muss sich auf diese Weise mit ihren Ängsten und Einschränkungen auseinandersetzen. Beispielsweise kommt Fausta dort beinahe täglich mit fremden Männern in Kontakt, seien es Handwerker oder Lieferanten. Dort lernt sie jedoch auch Noé, den höflichen, einfühlsamen Gärtner des Hauses kennen. Die beiden verbindet ihre gemeinsame Sprache Quechua, die Freude an Pflanzen und die Tatsache, dass beide in Manchay wohnen. Mit der Zeit beginnt Fausta ihm zu vertrauen und romantische Gefühle für ihn zu entwickeln.

Kurz nach dem Tod ihrer Mutter beginnt Fausta für Aída, einer wohlhabenden, in jüngeren Jahren sehr erfolgreichen Pianistin, als Hausmädchen zu arbeiten. Diese steht kurz vor ihrem alljährlichen Konzert zu dem die gesamte gesellschaftliche Oberschicht aus Lima erscheinen wird. Aída fehlt es jedoch an jeglicher Kreativität und sie scheint nervlich am Ende zu sein. In Fausta, die zur eigenen Beruhigung improvisierte Lieder auf ihrer Muttersprache Quechua singt, erkennt Aída ihre Chance und bietet einen Pakt an: Für jedes gesungene Lied bekommt Fausta eine Perle einer wertvollen Halskette, sobald sie die Kette vervollständigt, schenkt sie ihr die Perlenkette. 
Es kostet Fausta viel Überwindung, in einer ungewohnten Umgebung auf Spanisch zu singen. Für sich allein singt sie lediglich auf Quechua, der Sprache, die sie mit ihrer Mutter verbindet. Dennoch überwiegen ihre Not und der Wunsch, die eigene Mutter nach Hause zu bringen. Somit akzeptiert sie die Vereinbarung und beginnt für Aída, einer Frau, der sie nicht vertraut, zu singen offenbart ihr ihr Inneres. Aída macht sich die vielversprechenden, exotischen Klänge aus den Anden zu Nutze und kopiert schamlos eines von Faustas selbst komponierten Liedern namens La sirena. Auf dem Heimweg von ihrem glorreichen Konzertes, das ganz Lima begeisterte, wirft Aída die hilflose, völlig verängstigte Fausta nicht nur aus dem Auto, sie weigert sich auch ihren Pakt einzuhalten. Fausta bekommt weder die Bezahlung für einen Monat Arbeit, noch die versprochenen Perlen.

Verzweifelt und wütend zugleich, findet Fausta nach Máximas Hochzeit den Mut ohne Begleitung nach Lima zu gehen und die ihr zustehenden Perlen zu holen. Sie schleicht sich in das Anwesen, während Aída noch schläft. Fausta kriecht auf allen Vieren in das Schlafzimmer der Pianistin und sammelt die Perlen einzeln vom Boden auf. Mit den Perlen in der Hand verliert Fausta vollkommen entkräftet im Innenhof das Bewusstsein. Noé entdeckt sie am Eingangstor auf dem Boden liegend und trägt sie auf dem Rücken ins Krankenhaus, wo sich Fausta endlich die wurzelschlagende Kartoffel entfernen lässt.

Im Krankenhaus wartet ihr Onkel an ihrem Bett, bis sie aufwacht und ihm die Perlen in ihrer Hand zeigt (Screenshot Nr. 1). Dank dieser Perlen hat sie die nötigen Mittel, um ihre Mutter im Heimatdorf zu beerdigen. Auf dem Weg dorthin sieht sie das Meer und beerdigt ihre Mutter an diesem friedlichen Ort, weit weg von allen traumatischen Erinnerungen in den Anden.

Am Meeresufer finden Fausta und Perpetua die lang ersehnte Erlösung. Fausta ist mit dem Rücken zur Kamera gewandt. Der Zuschauer kann erkennen, dass sie sichtlich entspannter ist. Ihr Körper wirkt nicht mehr verkrampft, ihre Schultern hängen locker hinunter und sie atmet ruhig. Faustas Blick ist auf das rauschende Meer gerichtet. In dieser ruhevollen Umgebung, abgeschirmt von der Außenwelt, verweilt sie einige Momente und steht schweigend neben dem am Boden liegenden Leichnam ihrer Mutter. Die letzten Worte zu ihrer Mutter sind: „Mira el mar, ma. Mira el mar“ (La teta asustada 2009: 1.27.44- 1.29.06). Der Schluss ist geprägt von einer starken Symbolik, was weiter unten im Unterkapitel 4.5 genauer diskutiert wird. Noé stellt eine blühende Kartoffelpflanze vor Faustas Tor. Fausta atmet den Duft der Pflanze ein und lächelt. Mit dieser Szene endet der Film.

Nach dieser Zusammenfassung der Handlung, wird sich im nächsten Unterkapitel mit den 
Figuren und den Figurenkonstellationen befasst.

\subsection{Figuren und Figurenkonstellationen}

„Der Film als ein audiovisuelles Medium tendiert zum Sichtbaren, zum Äußerlichen und muß [sic] einen besonderen Aufwand betreiben, um Inneres - Gedanken, Gefühle, mentale Zustände usw. einer Person - zu gestalten“ (Faulstich 2002: 95).

Zur Beschreibung der Figuren und ihrer Handlungsbeziehungen ist das Aktantenmodell von Algirdas Julien Greimas (1966) hilfreich. Er nennt darin verschiedene Aktanten, die in unterschiedlichen Relationen zueinander stehen können. In jeder Handlung gibt es das Subjekt, das ein Objekt anstrebt oder begehrt und dabei von einem Gegner gestört oder von einem Helfer gefördert wird. (vgl. Kim 2002).

Fausta ist die Protagonistin und das Subjekt der Filmhandlung.

[Sie ist das] Wahrnehmungszentrum im Film, die Schlüsselfigur, die Klammer, die alles zusammenhält [...] Aufgrund [ihrer] Rolle als Wahrnehmungs- und Bedeutungszentrum im Film wird auf [ihre] Gestaltung in aller Regel Wert gelegt, eben um die notwendige Glaubwürdigkeit und zugleich Attraktivität zu erzeugen (Faulstich 2002: 95/ 96, Anm. der Verfasserin).

Ihre Präsenz dominiert im ganzen Film, sie ist in beinahe jeder Sequenz des Films zu sehen. Fausta Huamán ist eine junge Migrantin, die ihre ländliche Heimat in den Anden verlassen musste. Ihre Beweggründe waren die Suche nach Sicherheit und Unterstützung im Kreise der Familie des Bruders ihrer Mutter. Sie leidet an la teta asustada, einer Traumatisierung, die durch die Muttermilch ihrer, während der Schwangerschaft vergewaltigten, Mutter an sie weitergegeben wurde. Es ist eine

[e]nfermedad que se manifiesta en el cuerpo de Fausta con sangrados por la nariz o desmayos cuando se enfrenta a situaciones límite o ataques de pánico, pero también en su personalidad convirtiéndola en un ser retraído, temeroso, desconfiado, hermético e insensibles con su familia y el resto de personas, sobre todo con los hombres que son para ella potenciales agresores sexuales. Es como si no tuviera alma (Laura Atanacio 2016: 86).

Infolge dieser Krankheit ist die introvertierte Fausta unfähig, mit fremden Personen zu kommunizieren, sie vermeidet generell Gespräche mit Menschen und bevorzugt es zu schweigen. Ihr Bewusstsein wird stark von ihren sozialen Ängsten kontrolliert, was dazu führt, dass sie das Haus nicht alleine verlassen kann. Sie ist in ständiger Begleitung von Familienmitgliedern, denen sie meistens mit einem geringen Abstand folgt. Wenn es die Gegebenheiten zulassen, läuft sie eng an Mauern vorbei und blickt sich in kurzen Abständen über die Schulter, um mögliche Gefahren sofort zu entdecken (vgl. Laura Atanacio 2016). 
„[Y] al llegar a una esquina corre de prisa por temor a que las almas le quiten la suya“ (Laura Atanacio 2016: 71). Außerdem äußert sich diese Angst vor körperlichen Übergriffen durch eine konstante physische Anspannung, das heißt Fausta wirkt sehr steif oder starr mit ihrer etwas gebeugten Körperhaltung, den eingezogenen Schultern und den geballten Fäusten. Fausta greift aber auch zu drastischeren Maßnahmen, um sich vor möglichen Übergriffen zu schützen. Sie hat sich zur Abwehr eine Kartoffel in die Vagina geschoben, die zum einen eine Vergewaltigung unmöglich machen soll und zum anderen beim Angreifer großen Ekel erregen soll, denn ihr zufolge kann nur etwas Ekelhaftes einen ekelhaften Menschen abwehren.

Faustas Gesicht strahlt Traurigkeit, sowie Verzweiflung und permanentes Leiden aus. gibt Es gibt kaum mimische Veränderung in ihrem Gesicht. Trotz der Zurückhaltung der emotionalen Expressivität liegt eine starke Betonung auf ihren Augen, in denen sich ihre Gefühle wiederspiegeln. Sie sind häufig vor Angst weit geöffnet und glasig. Als Zuschauer erkennt man keine Persönlichkeit in ihr, es ist unmöglich erkennbar, wie sie wirklich ist, weil ihr ganzes Wesen aufgrund der Leiden verdeckt bleibt. Sie ist klein und schlank. Sie kann als archetypische Quechua-Schönheit beschrieben werden. Mit den langen, glänzenden, pechschwarzen Haaren, den schwarzen Augen, ihren hohen Wangenknochen und einer von der starken Sonne in den Anden gebräunte Haut entspricht die Darstellerin Magaly Solier dem westlich geprägten Bild einer exotischen Frau. Fausta trägt keine traditionelle Quechua-Tracht. Genaueres zu Faustas Kostüm und Maske wird im nächsten und wichtigsten Unterkapitel der Analyse folgen. Der individuelle Charakter der Schauspielerin spiegelt sich auch in der Figur der Fausta wieder. „,[N]atürlich tragen auch die schauspielerischen Fähigkeiten des jeweiligen Darstellers und seine gestalterische Interpretation wesentlich zur Charakterisierung einer Figur bei“" (Faulstich 2002: 100). Wie bereits erwähnt kann sich der minimalistische Filmstil auch durch die Sparsamkeit der Gesichtsausdrücke und Gesten der Charaktere kennzeichnen. Dies wird bei Fausta bis ins Äußerste getrieben. Sie verweigert sogar jeglichen Augenkontakt.

Die anfangs noch von Ängsten geplagte Fausta entwickelt sich im Laufe des Films hin zu einer selbstbewussteren Frau, die sich emanzipiert. Fausta ist daher ein mehrdimensionaler Charakter. Zu Beginn macht sich ihre Traumatisierung stark bemerkbar, sie bekommt mehrmals Panikattacken und Ohnmachtsanfälle in Stresssituationen. Ihr Handeln wird durch ihre Ängste kontrolliert. So zum Beispiel in Sequenz 3, als sie ihrem Onkel über den Tod ihrer Mutter berichtet. Sie bekommt Nasenbluten und fällt in Ohnmacht. Nasenbluten und 
Atemnot bekommt sie auch, als sie Aída das erste Mal in Sequenz 21 kennenlernt. Aída bohrt in dieser Szene ein Loch in die Schlafzimmer Wand, um ein weiteres Bild aufzuhängen. Dabei soll Fausta ihr behilflich sein. Während sie die Bohrmaschine bereithält, sieht sie zuerst ihr eigenes Spiegelbild in dem Glas eines Bildes und erkennt erst dann, um welche Fotografie es sich handelt. Die Fotografie zeigt einen Mann des peruanischen Militärs, womöglich einen Offizier. Auch Aída ist in dieser Szene ähnlich gekleidet. Sie trägt eine lockere Buntfaltenhose, die in hohe Lederstiefel gesteckt ist, außerdem ein braunes Oberteil und eine wertvolle Perlenkette, die mehrmals um ihren Hals gewickelt ist. Diese Situation ist zu viel für Fausta, denn unterbewusst verknüpft sie das Bild des Offiziers und die Kleidung ihrer Arbeitgeberin mit der Vergangenheit ihrer Mutter. Sie rennt in die Küche, wäscht sich und singt zu ihrer eigenen Beruhigung.

Zudem Fausta bekommt zunehmende körperliche Beschwerden aufgrund der Kartoffel, denn diese schlägt Wurzeln, die sie regelmäßig abschneiden muss, um noch stärkere Schmerzen zu vermeiden. Trotz des Abschneidens der Triebe müssen die Schmerzen immer noch unerträglich sein, denn Fausta bekommt in Sequenz 62 während einer Hochzeitsfeier ebenfalls einen Ohnmachtsanfall und reibt sich den Unterleib, nachdem sie wieder zu sich kommt. Die einzigen Figuren, die Fausta versuchen, umzustimmen und sie dabei unterstützen, ihr Leben zu ändern, sind ihr Onkel und Noé. Beide stehen Fausta zur Seite und helfen ihr eine wichtige Entscheidung zu treffen. Auf die Beziehung zwischen Noé und Fausta wird etwas weiter unten noch detaillierter eingegangen. Noé überzeugt sie Stück für Stück in mehreren Gesprächen. Die Gespräche handeln nicht explizit von der Maßnahme, zu der Fausta gegriffen hat. Das Thema der Kartoffel wird lediglich angespielt. Noé stellt Fausta verschiedene Pflanzen vor. Dabei erwähnt er in Sequenz 61, eher beiläufig, dass die Kartoffelpflanze billig ist und keine schönen Blüten bekommt. Daher wird sie nicht in Aídas Garten angepflanzt. Nach dieser Aussage nimmt Fausta einen traurigen, enttäuschten Gesichtsausdruck an. Daraus kann schlussgefolgert werden, dass Fausta sich von dieser Aussage persönlich angegriffen fühlt.

Der Onkel greift, in der Nacht nach Máximas Hochzeit, zu einer drastischeren Maßnahme, um Fausta zu überzeugen. Bereits betrunken, versucht er bei Fausta den Überlebenswillen zu wecken, indem er ihr den Mund zuhält. Fausta wird so aus dem Schlaf gerissen und rennt davon. Doch es scheint so, als hätte dieses Erlebnis sie aufgerüttelt. Sie ist nun nicht mehr die panische Fausta, sondern nimmt sich, was ihr zusteht. Außerdem überwindet sie ihre Ängste und lässt die Kartoffel aus ihrer Vagina entfernen. Die Kartoffel steht sinnbildlich 
für die Traumatisierung. Daher ist die Entfernung der Kartoffel der wichtigste Schritt des Befreiungsprozesses. Als Fausta endlich die finanziellen Mittel aufgebracht hat, kann sie sich von ihrer Mutter verabschieden und ihre Vergangenheit hinter sich lassen. In Sequenz 83 und 84 wird Faustas Gesicht gezeigt und die Erleichterung darüber, endlich die Mutter beerdigen zu können, die sich darin ausbreitet. Sie genießt den Fahrtwind, der ihr durch die Haare weht und ist sichtlich begeistert, als sie das Meer entdeckt.

Figurenkonstellationen machen einen Großteil der Dramaturgie aus und klassische „Rollen sind oft soziale Verhaltensschemata und Berufe“ (Faulstich 2002: 96), die Bezüge zur aktuellen politischen und gesellschaftlichen Situation aufweisen. Typische Figurenpaarungen, die in La teta asustada auftreten, sind die Arme und die Reiche, die Gute/ das Opfer und die Böse/ die Täterin, außerdem noch das Liebespaar, sowie die Hilfsbedürftige und der Helfer.

Die Beziehung zwischen Fausta und ihrer Mutter Perpetua ist überaus eng und lässt sich unter anderem an der liebevollen Art und Weise mit der die Tochter ihre Mutter pflegt, ausmachen. Fausta ist aufgrund der intergenerationellen Traumatisierung an ihre Mutter gebunden. Obgleich Perpetua schon zu Beginn des Films stirbt, ist sie trotzdem eine omnipräsente Figur (vgl. Laura Atanacio 2016). Da sich weder Fausta, noch Lúcido ein würdevolles Begräbnis in Perpetuas Heimat - der Gegenstand oder das Ziel aller Handlungen, die von Fausta ausgehen - leisten können, bewahrt Fausta den mumifizierten Körper ihrer Mutter lange Zeit, genau genommen bis zur 84. Sequenz, auf. Die Mumifizierung des Körpers war in Zeiten des Konfliktes nötig, um den Behörden Beweise für die Existenz und die Ermordung der ländlichen Bevölkerung zu liefern. Damals gab es weder Geburtsurkunden oder Fotos, noch richtige Begräbnisse. Im Film findet die Mumifizierung der Mutter in Sequenz 14 statt (Screenshot Nr. 2). Fausta schläft danach weiterhin neben ihr, streicht ihr Haar glatt und singt ihr vor, ganz so als würde sie noch leben. Fausta fühlt sich von anderen missverstanden und wendet sich daher weiter an ihre Mutter, sie kann sich noch nicht von ihr lösen. Perpetua ist jedoch nicht nur körperlich anwesend, ihr Andenken und ihre Geschichte sind allgegenwärtig, auch wenn es innerhalb der Familie nicht direkt angesprochen wird. In Anbetracht dieser furchtbaren Vergangenheit wird Perpetua nicht als Opferfigur dargestellt, sie wird stattdessen als ein Individuum gezeigt, das darum kämpft nicht vergessen zu werden und mithilfe ihres Gesangs eine Art kollektives Gedächtnis kreiert und somit auch für andere Frauen spricht, die wie sie leiden mussten. Ihr Name Perpetua, zu Deutsch Immortelle oder Unsterbliche, wurde daher auch 
symbolisch gewählt, denn ihre Geschichte und diejenige zahlreicher anderer QuechuaFrauen darf nicht vergessen werden.

Die Bewohner von Manchay werden als eine fröhliche und aktive Gesellschaft gezeigt, die stetig wächst, tolerant und heterogen ist. Sie feiern gerne Feste, vor allem Hochzeiten, die Leben in diese triste, sandige Landschaft bringt, wo spielende Kinder, das Rauschen des Küstenwindes und gelegentlichen Verkehrslärm von der Landstraße durch die Anden zu hören sind.

Die vielköpfige Familie von Fausta setzt sich zusammen aus dem Onkel Lúcido, seiner Ehefrau Carmela und ihrer gemeinsamen und einzigen Tochter Máxima. Weitere Cousins und Cousinen von Fausta - Jonathan, Melvin, Chicho und Severina - außerdem eine Großmutter, eine kleine Nichte, sowie ein Neffe bilden den Rest der Familie. Nach dem grausamen Tod, den Faustas Vater ertragen musste, flohen Perpetua und Fausta aus ihrer Heimat. Zum einen um von weiterer Gewalt verschont zu werden und zum anderen um sich einen Lebensunterhalt zu sichern. Im Familienbetrieb des Onkels, der Hochzeitsplanungsgesellschaft, findet Fausta einen Weg um ihrem Onkel etwas Dankbarkeit entgegenzubringen, angesichts der Tatsache, dass er sie beide auf seinem Grundstück wohnen lässt und mitversorgt.

La familia Huamán, incluida Fausta, trabaja en su micro empresa dedicada a la organización de fiestas de matrimonio para familias de su mismo nivel socioeconómico. Bodas Chic, como así se llama, se encargan de todas los detalles para los recién casados, desde el catering hasta la animación, del registro fotográfico al decorado y demás (Laura Atanacio 2016: 63).

Innerhalb dieser Familie lassen sich traditionelle Geschlechterrollen ausmachen. Die Frauen beschäftigen sich mit dem Haushalt, zu ihren Aufgaben gehören unter anderem kochen, nähen, putzen und die Kindererziehung. Die jungen Männer - mit einer Ausnahme verbringen die meiste Zeit mit angenehmen Beschäftigungen, wie Musik hören oder Videos drehen. Lúcido ist der einzige, der sich körperlichen Aufgaben widmet. Er gräbt z.B. ein Loch im sandigen Hof, um einen Pool zu bauen. Die Ausnahme, von der eben die Rede war, ist der homosexuelle oder transsexuelle - dies ist hier nicht ganz klar zu deuten - Cousin Jonathan. Er übernimmt Aufgaben, die traditionellerweise eher Frauen zugesprochen sind, wie das Aufpassen auf das Baby. „,[A]demás, trabaja como cosmetólogo, una profesión asociada a lo femenino y por antonomasia a la población trans masculina“ (Laura Atanacio 2016: 63).

Fausta hat eine gute Beziehung zu ihrem Onkel, er kümmert sich fürsorglich um sie, wenn sie einen ihrer Ohnmachtsanfälle bekommt, begleitet sie ins Krankenhaus und zeigt 
Mitgefühl. Er hat somit eine helfende Funktion. Auch wenn er ihr wohlgesonnen ist und sie im täglichen Leben unterstützt, akzeptiert er nicht, dass Fausta ihrem Körper Schaden zufügt und ihr junges Leben vergeudet. Zu Beginn weiß er nicht zu welchen Maßnahmen Fausta gegriffen hat, um sich vor sexuellen Übergriffen zu schützen. Dies erfährt er erst in Sequenz 5, durch den behandelnden Arzt, der ihn darüber informiert, dass Fausta ein tubérculo in die Vagina eingeführt hat und daher ihre Beschwerden herrühren. Der Onkel schildert dem Arzt, dass Fausta an la teta asustada leidet, doch dieser streitet jegliche Existenz dieser Krankheit ab. Lúcido ist sichtlich schockiert und spricht kein Wort mit Fausta, die im Bus nach Hause mehrere Anläufe startet, ihm ihre Gründe zu erläutern. In einigen Momenten kann bei ihm ein gequälter Gesichtsausdruck beobachtet werden, was darauf hindeutet, dass das Thema ihm etwas unangenehm ist. Fausta vermeidet den Augenkontakt. In der Nacht nach der Hochzeitsfeier seiner Tochter versucht Lúcido, getrieben vom Alkohol, seine Nichte ihren Überlebensgeist zu wecken, indem er der schlafenden Fausta den Mund zuhält, sodass sie keine Luft bekommt und aus dem Schlaf hochschreckt. Diese grobe Methode scheint doch gefruchtet zu haben. Die aufgeschreckte Fausta rennt den ganzen Weg von Manchay zum Anwesen in der Stadt, um sich die Perlen, die ihr zustehen, von Aída zu holen.

Auch die anderen Familienmitglieder, ebenso Helfer, kümmern sich um sie. Ihrer Tante Carmela hat sie unter anderem den Arbeitsplatz zu verdanken. Die Gleichaltrigen begleiten sie an verschiedene Orte, denn Fausta geht niemals allein außer Haus. In Sequenz 12 begleitet der Cousin Chicho sie zu drei verschiedenen Bestattungsinstituten und übernimmt dort das Reden. Auch Severina, die schwangere Cousine, begleitet sie in Sequenz 29, zur Busstation, um dort zu fragen, ob es gestattet ist einen Sarg im Bus zu transportieren. Jonathan versucht sie in Sequenz 36 mit seinem Bekannten Amadeo zu verkuppeln. Alle drei wissen nicht wirklich wie sie mit Faustas Krankheit umgehen sollen und auch Fausta verhält sich teilweise sehr egoistisch und stellt ihr eigenes Bedürfnis nach Sicherheit vor die Bedürfnisse anderer. Máxima ist sichtlich missgelaunt, denn sie ist mit ihrer bevorstehenden Hochzeit beschäftigt. Darum ,siente rivalidad por Fausta debido a la sobre atención que su padre le presta" (Laura Atanacio 2016: 63). Bereits in der dritten Sequenz, als Fausta ihren Onkel über Perpetuas Tod benachrichtigen will, beschwert sich Máxima über die Länge ihres Hochzeitsschleiers, der bereits an die 3 Meter misst, ihr jedoch immer noch zu kurz ist und sich daher sehr egoistisch, wie ein quengelndes Kind aufspielt.

Noé, der Gärtner auf Aídas Anwesen in Lima, kann aufgrund der gemeinsamen Herkunft und Sprache eine Beziehung des Vertrauens zu Fausta aufbauen, ist demnach ebenso ein 
Helfer an Faustas Seite.

For her to trust Noé takes a considerable amount of time, but as Fausta is able to gain enough confidence, their interactions indicate a drastically different relationship as Fausta has with her family and her employer. Noé never demonstrates anxiety towards Fausta, nor does he question her beliefs or patronizes her as Aída does (Vilchez 2014: 10).

Er ist einer der wenigen, mit dem Fausta redet und mit dem sie ihre Gefühle oder Gedanken teilt. Durch ihn gewinnt sie Selbstvertrauen und beginnt sich von ihrem alten Leben zu lösen.

[I]t is Noé (the biblical rescuer) who seems to wake her up from her stupor. He (Efraín Solis) displays kind manners, speaks Quechua with her, and offers to her wise comments. Like her uncle, Noé cares for Fausta but places himself at a distance, respecting her space, but being there when Fausta needs him. Noé tells Fausta that 'death is the only part of your existence that is predetermined,' and that he does not plant potatoes because they are too common and provide almost no flowers (Maseda 2016: 18).

Wie bereits erwähnt, entwickelt Fausta eine Mischung aus väterlichen und romantischen Gefühlen für Noé, die aber im Film lediglich angedeutet bleiben und nicht näher gezeigt werden. Im ursprünglichen Drehbuch war die Beziehung zwischen den beiden eindeutig romantischer Natur.

[E]l personaje Noé es un hombre joven de origen rural andino, casi de la misma edad de Fausta. Asimismo, en una escena del guión [sic] y que no aparece en el montaje final, Fausta le confiesa a su primo Jonathan, ante los reclamos de éste por haber rechazado la compañía de Amadeo que no es él que le gusta (Laura Atanacio 2016: 110).

Die Rolle der Antagonistin oder Gegnerin übernimmt Aída. Aída ist eine erfolgreiche Pianistin spanischer Abstammung. Wohnhaft ist sie in einem komfortablen Anwesen mit einer wunderschönen Grünanlage. Wie bereits erwähnt, hat sie Verbindung zum Militär. Aída ist eine elegante, aber ernste Frau, die gleich vom ersten Moment an unsympathisch wirkt. Sie hat einen mürrischen Gesichtsausdruck und wirkt streng und gebieterisch. Sie ist gut gekleidet und stellt optisch das exakte Gegenteil zu Fausta dar. Sie ist groß, blond und besitzt europäische Gesichtszüge.

Die Tatsache, dass sie Fausta immer beim falschen Namen nennt, beweist ihr Desinteresse an der dienenden Unterschicht, die sie ganz offensichtlich sich selbst unterordnet. Bei ihrer Einstellung schenkt sie Fausta Hygieneartikel - einen Nagelknipser, Deodorant, einen Spiegel und einen Kamm - denn sie möchte sicher stellen, dass ihr neues Dienstmädchen sauber zur Arbeit erscheint. Sie befindet sich in einer kreativen Krise, einer Blockade, die sie an der Komposition eines in näherer Zukunft geplanten Konzerts hindert. Ihre Frustration darüber bringt sie sogar dazu ihr wertvolles Piano aus dem bunten Bleiglasfenster zu stoßen. Dabei zerstört sie nicht nur das Klavier, sondern auch dieses historische Buntglas, welches an ein Kirchenfenster erinnert. Als sie herausfindet, dass Fausta Lieder mit neuen 
Thematiken und unbekannten Melodien singt, nutzt sie Faustas Notlage aus und macht ihr ein Angebot, das sie nicht ablehnen kann. Für jedes gesungene Lied verspricht sie ihr eine echte Perle. Diese könnte Fausta verkaufen, um ihrer verstorbenen Mutter das lang ersehnte, würdevolle Begräbnis zu ermöglichen. Daher hat sie auch zum Teil eine unterstützende Funktion für Faustas Vorhaben, sie ist also nicht nur ihre Gegnerin, sondern auch Helferin. Sie ist einzig und allein auf ihren eigenen Vorteil aus und besitzt keine Skrupel, Faustas missliche Lage auszunutzen und sie sogar noch zu betrügen und ihr die versprochenen Perlen nicht zu geben:

Getting closer to the conclusion, the terrible upper-class woman essentially steals what Fausta has produced for her own recital, without giving credit or a thank you. Towards the end of the film, and slowly demonstrating that she is gaining more of her own voice and self, Fausta makes a remark about the reception of the recital - the applause is truly hers [Spanisch: Les gustó mucho, ¿no?] - which leads to her being dropped off on the side of the highway (Vilchez 2014: 11, Anm. Der Verfasserin).

Aída lässt sie mitten in der Nacht auf einer stark befahrenen Straße stehen, wie einen ausgesetzten Hund und das, obwohl sie sich Faustas Ängsten bewusst ist. Doch sie will sich die Wahrheit über ihren Erfolg nicht eingestehen und schon gar nicht Faustas Talent bestätigen.

Zum Schauspiel und der Inszenierung der Darsteller und Darstellerinnen ist zu sagen, dass diese minimal gehalten werden: Sie nutzen keine großen, ikonischen Gesten. Außerdem dominiert die mimische und gestische Bewegungslosigkeit. Claudia Llosa hat wohl zum Teil auf Laien zurückgegriffen, um ihre Rollen zu besetzen. Diese strahlen Authentizität und Natürlichkeit aus. Im Anschluss an diese Figurenanalyse folgt die Analyse der filmischen Gestaltungsmittel in La teta asustada.

\subsection{Filmische Gestaltungsmittel}

Das folgende Kapitel analysiert die eingesetzten filmischen Gestaltungsmittel und bezieht sich dabei stets auf das erstellte Sequenzprotokoll, das im Anhang unter der Kapitelnummer 7.1 zu finden ist. Man wird sich, der Reihenfolge im Sequenzprotokoll nach, mit den wichtigsten Elementen, die sogenannten Bauformen der filmischen Gestaltung befassen: Anzahl der Sequenzen und Average Shot Length (ASL), Drehort, Kostüm, sowie Maske der Figuren. Außerdem werden Filmmusik und Geräusche oder Momente der Stille analysiert. Danach folgen Kameraführung, genauer gesagt Einstellungsgröße und 
Kamerabewegung, Farben und Licht, sowie die Mise-en-scène oder Komposition des Bildes, die aber in Verbindung mit dem Drehort analysiert wird. Wie dieses Sequenzprotokoll erstellt wurde, wurde bereits weiter oben im Unterkapitel 4.1 präzisiert. Zuerst werden die Merkmale der audio-visuellen Gestaltung beschrieben, um später im nächsten Unterkapitel deren Wirkung und Bedeutung zu klären.

\section{Anzahl der Sequenzen und ASL}

Wie aus dem realisierten Sequenzprotokoll ersichtlich ist, besteht der Film aus 86 Sequenzen. Wie bereits erläutert, ist die entscheidende Einheit für die Einteilung der Sequenzen in diesem Protokoll der Ortswechsel. Das heißt, jede neue Sequenz beginnt mit einem Ortswechsel. Jede Sequenz besteht aus mehreren Einstellungen. Mit knapp über 300 Einstellungen bei einer Filmdauer von 90 Minuten und 20 Sekunden unterscheidet sich $\mathrm{La}$ teta asustada deutlich von anderen Filmen, die im Durchschnitt deutlich mehr Einstellungen aufweisen. Die durchschnittliche Einstellungslänge oder ASL beträgt ca. 18 Sekunden, demnach gehört er eindeutig zu den langsamer geschnittenen Filmen. Vor allem in Hollywood hat sich die durchschnittliche Einstellungslänge in den letzten Jahrzehnten drastisch verringert. Die ASL in den Filmen von Steven Spielberg beträgt beispielsweise 6,5 Sekunden und die ASL des Regisseurs Michael Bay beträgt lediglich 3,0 Sekunden (web. Nedomansky 2017; 23.08.2018).

\section{Drehort mit Mise-en-scène}

Das nächste zu untersuchende Element ist der Drehort bzw. die Drehorte. Der Drehort ist der Standort des Sets, an dem ein ganzer Film oder lediglich eine Szene gefilmt wird. In $L a$ teta asustada handelt es sich um reale Standorte und keine Filmstudios. In La teta asustada gliedern sich die Drehorte grob in zwei Kategorien. Zum einen sieht der Zuschauer den ruralen Raum, der die Komfortzone von Fausta wiederspiegelt. Zum anderen gibt es den urbanen Raum, hier die Hauptstadt Lima, die, wie in vielen Teilen der Welt Zentrum der Politik, Wirtschaft und kulturellen Innovation ist, aber auch den größten Stressfaktor für die Protagonistin in La teta asustada repräsentiert, da sie sich außerhalb ihrer Komfortzone bewegen muss und mit zahlreichen Gefahren konfrontiert wird. Beide Räume unterscheiden sich grundlegend voneinander.

Betrachtet man den ruralen Raum, kann man beobachten, dass es sich um das stetig wachsende Armenviertel Manchay handelt, das sich im Einzugsgebiet der Stadt Lima 
befindet. Manchay erstreckt sich über eine Fläche von $11 \mathrm{~km}$ und befindet sich im Distrikt Pachacamac, welcher an die Distrikte Molina und Cieneguilla angrenzt. Viele Einwohner, die hier leben, mussten damals in Zeiten des Terrorismus, provoziert durch die kommunistische Partei Sendero Luminoso, auf der Suche nach Sicherheit und Zukunftsperspektiven aus ihren Heimatdörfern in den Anden Richtung Hauptstadt fliehen. Seit den 90er Jahren gibt es einen jährlichen Zuwachs von durchschnittlich 1000 Familien. Die genaue Einwohnerzahl ist schwer festzulegen, sie wird auf um die 100.000 Personen geschätzt. Der Wohnungsmangel in Lima und der niedrige sozioökonomische Hintergrund verwehren vielen Bürgern aus den unteren Schichten Perus den Zugang zum Stadtinneren. Daher bleibt den Inlandsmigranten aus den Andengebieten nichts Anderes übrig als sich außerhalb der Hauptstadt, zusammen mit ihren Gleichgestellten, in provisorischen Häusern aus Holz und Luftziegeln anzusiedeln. Die Häuser werden auf einem Boden gebaut, der nicht für den Häuserbau geschaffen ist, denn er besteht zum größten Teil aus Sand. Oft leben die Bewohner der Vororte in größter Armut, ohne Elektrizität und fließendes Wasser. Häufig teilen sich mehrköpfige Familien einen einzigen Wohnraum, in dem sie schlafen, kochen, essen und leben. Jugendliche organisieren sich in kriminellen Banden, um zu überleben und träumen von einem besseren Leben. 36\% der Einwohner sind arbeitslos und 53\% leben von Gelegenheitsjobs, die ebenfalls keine Stabilität und finanzielle Absicherung bieten. Außerdem gehört die Region um Manchay zu den gefährdetsten Gebieten des Landes, was schwerwiegende Erdbeben betrifft (web. https://manchayperu.com; 03.07.2018).

Das Zuhause von Fausta und ihrer Familie besteht aus einem großen, sandigen Hof, der durch eine Mauer und ein großes Metalltor von anderen Grundstücken isoliert wird. In diesem Hof steht ein provisorisches Betonhaus, indem sich Fausta und ihre Mutter ein Zimmer teilen. Dieses Zimmer, Drehort A., sieht man in den Sequenzen 2, 9, 11, 14, 37, 46, 53, 55 und 72. In Sequenz 2 (Screenshot Nr. 3/ 4) sieht man dieses Zimmer, in dem ein altes, abgegriffenes Bettgestell vor einer bunten Tapete mit Rosen steht. Der Lack des Gestells und die Tapete blättern bereits ab. Ein Jesuskalender hängt an der Wand. Der Zuschauer erkennt am Zustand des Bettes und der Tapete, dass sie nicht zur oberen, wohlhabenden Schicht in Peru gehören. Durch das Fenster ohne Fensterscheibe wird eine weitgefächerte Sicht über das schlichte, ärmliche Viertel geboten, das sich über die Berge ausbreitet. Es kann nicht eindeutig festgemacht werden, wie weit sich das Stadtviertel erstreckt. Die Anden bilden im verschwommenen Hintergrund eine natürliche Grenze. Wenige Meter von Faustas Behausung befindet sich ein kleines, einfaches Steinhaus mit einem Dach aus Holz und 
Plastikplanen, in dem der Onkel mit seiner Frau und der gemeinsamen Tochter wohnt. Ein großer Plastiktisch und Stühle, zusammen mit bunten Möbelstücken stehen davor (Sequenz 3, Screenshot Nr. 5/ 6). Der Innenhof stellt den Drehort B. in den Sequenzen 3, 10, 36, 45, 52, 66, 85 und 86 dar.

Die Siedlung Manchay stellt noch in weiteren Sequenzen des Films den Drehort dar. Dabei erinnert Manchay häufig an ein Labyrinth (Screenshot Nr. 7/ 8). Das Labyrinth funktioniert hier als Mittel der Figurencharakterisierung von Fausta. Es ist ein Labyrinth, aus dem Fausta nur schwer entkommen kann, da sie sich selbst darin gefangen hält. Es symbolisiert die ausweglose Situation, in der sie sich befindet. Außerdem ein Labyrinth, in dem es immer wieder ähnliche oder dieselben Elemente zu sehen gibt und somit die Monotonie des Alltagslebens dargestellt wird. Ein wiederkehrendes Element ist die lange, steile, gefühlt endlose Treppe (Screenshot Nr. 9), die von einer Landstraße nach Manchay führt (Sequenzen 26, 30, 43). Außerdem wird der Weg von dieser Treppe durch das Viertel, vorbei an unzähligen, nicht fertig gestellten Rohbauten inmitten einer staubig-kargen Landschaft in den Sequenzen 8, 44 und 76 gezeigt. Sich wiederholende Elemente sind ebenso die Bestandteile der zahlreichen Hochzeiten wie beispielsweise die Hochzeitstreppe, von der die Brautpaare herabsteigen und die farbenfrohe Dekoration (Sequenzen 27, 28, 62, 73, 74). Die Hochzeitsdekoration schafft einen starken Kontrast zur kargen, monochromen Landschaft in und um Manchay herum. Man sieht im Übrigen noch den Wohnraum des Onkels und seiner Familie in den Sequenzen 13 und 71, dabei wirkt er sehr überladen und vollgestellt mit Alltagsgegenständen. Außerdem ist dieser enge Wohnraum gleichzeitig der Arbeitsraum ihrer Hochzeitsplanungsgesellschaft, in der die gesamte Familie aushilft. Das heißt, in diesem Raum gibt es auch Dekoration, eine gigantische, mit Schleifen aus Zuckerguss verzierte Hochzeitstorte und Tauben, die aus einem Käfig in der Torte flüchten und wieder eingefangen werden müssen.

Die zahlreichen Hochzeiten, die sie planen, finden ebenfalls in Manchay statt. An dieser Stelle kann erwähnt werden, dass Claudia Llosa tatsächliche Paare engagiert hat, die ihre eigene Hochzeit nachstellen sollten (web: Nikolaidis 2010; 02.08.2018). Dabei kann in der Sequenz 62 ein für westliche Zuschauer sehr ungewöhnliches Phänomen beobachtet werden. Es handelt sich um die so genannte matrimonio civil comunitario, eine Massenhochzeit, die in Peru eine übliche Praxis darstellt. Dabei sparen die Paare erstens Geld und bekommen schneller einen Termin. Die Massenhochzeit im Film findet auf einem kahlen, sandigen Plateau in den Bergen statt (Screenshot Nr. 10). Im Hintergrund sind lediglich die Anden 
und ein klarer, blauer Himmel zu sehen. Die zahlreichen Brautpaare sitzen in Reihen auf verzierten Plastikstühlen. Andere Hochzeiten werden in den Sequenzen 27, 28 und 74 dargestellt. Dabei zeigt letztere die Hochzeit von Máxima, der Tochter des Onkels. Bei Máximas Hochzeit werden noch einmal die ärmlichen Verhältnisse deutlich hervorgehoben. Zwar werden in dem Sinne keine Kosten und Mühen für das Hochzeitskleid, die Dekoration und die Unterhaltung gescheut, trotzdem ist klar erkennbar, wie begrenzt ihre Möglichkeiten in dieser von der Hauptstadt Lima abgeschotteten Umgebung sind. Zum Beispiel wird eine Leinwand, die einen Wasserfall abbildet, genutzt, um einen ansehnlichen Hintergrund für die Hochzeitsbilder zu kreieren und die Baustelle im Hintergrund zu verdecken(Screenshot Nr. 11). Die Hochzeiten finden im Übrigen ausschließlich im Freien statt.

Im Gegensatz zur monotonen Umgebung von Manchay steht das urbane Umfeld der Hauptstadt Lima: Als größte, sowie politisch und wirtschaftlich wichtigste Stadt des Landes, vereint sie Elemente, welche in vielen Metropolen der Welt zu finden sind: Arbeitsplätze, verschiedene Lebensstile, mehrere Millionen Menschen aus unterschiedlichen Kulturen, Finanzen etc. In den Anden gelegen, ist sie Zuzugsraum für die arme Landbevölkerung und Binnenflüchtlinge, die Opfer des bewaffneten Konfliktes geworden sind. Auch allerlei andere Menschen aus jeglichen Städten und Regionen des Landes kommen in großer Zahl um Arbeit und Ausbildung zu finden. Dadurch herrscht in Lima ein reger Mix aus peruanischen Kulturen und Ethnien. Hinzu kommt die Anwesenheit der Regierung in Form des Parlaments und des Justizpalasts, sowie nationale und internationale Firmen. Daraus entsteht auch die reiche Oberschicht Perus, die hauptsächlich im Südwesten der Stadt, an der Küste angesiedelt ist, während die Unterschicht in Armut und Kriminalität die Ränder der Stadt bevölkert. Somit bildet das Zentrum Limas die Grenze zwischen Arm und Reich, wo sich alle Bevölkerungsschichten mischen. Lima ist ein Ort voller Energie, Farben und Sehenswürdigkeiten, der stark in Kontrast mit den einfarbigen, von der Sonne vertrockneten und eher trostlosen Vororten von Lima steht. An die 9 Millionen Menschen verkehren in Lima. Während es viele kulturelle Angebote gibt, ist Kriminalität, Unsicherheit und Gefahr ein großes Thema, weshalb sich die Oberschicht weitestgehend durch hohe Mauern vom Rest der Stadt abschottet. Bereits in den 80er Jahren initiierte eine Privatschule aus dem Stadtviertel Santiago de Surco den Bau einer Mauer, die die Schüler und Schülerinnen vor Attacken der Terrororganisation Sendero Luminoso schützen sollte. Die mit Stacheldraht besetzte Mauer aus Beton wurde auch später noch konstant erhöht und verlängert, um die wohlhabenden Stadtteile weiterhin von den Armenvierteln zu separieren (web. Ismar/ Cueva 
Sáenz 2017; 03.07.2018).

Als Fausta in Sequenz 4 nach ihrem, erstmals im Film zu sehenden, Ohnmachtsanfall im Krankenhaus aufwacht, tritt der Zuschauer das erste Mal mit dem urbanen Raum in Kontakt. Fausta liegt auf einem Gynäkologenstuhl in einem spärlich eingerichteten, im typischen krankenhausweiß gestrichenen Behandlungszimmer. Sie liegt auf dem Rücken und von der Decke scheint ihr ein grelles, flimmerndes Neonlicht ins Gesicht. Fausta wird durch einen Vorhang vor fremden Augen geschützt. Hinter diesem Vorhang befindet sich der andere Teil des Zimmers, in dem ein einfaches Büro für den behandelnden Arzt eingerichtet ist. Dieses Besprechungszimmer sieht man in Sequenz 5. Der Schreibtisch ist überladen mit Papieren, was darauf hindeutet, dass der Arzt viele Patienten betreuen muss. In der nachfolgenden Sequenz folgt Fausta ihrem Onkel durch die kahlen, weißen Krankenhausflure, ohne Sitzmöglichkeiten oder Schilder. Auch hier kommt das Motiv des Labyrinths zum Einsatz (Screenshot Nr. 12).

Der nächste zu analysierende Drehort in Lima ist das Herrenhaus von Aída, in dem Fausta zu arbeiten beginnt. Das Haus repräsentiert einen äußerst symbolträchtigen Ort. Dabei sind viele Gegensätze und Kontraste zwischen dem Garten/ Innenhof und dem Inneren des Hauses erkennbar. Der paradiesische Garten oder Innenhof, Drehort C., wird in den Sequenzen 16, 25, 31, 35, 41, 50, 56, 58, 60 und 79 dargestellt. Der Garten kann als goldener Käfig beschrieben werden, der alle, die sich darin befinden vor der lauten und chaotischen Außenwelt durch eine hohe Mauer und ein großes, braunes Garagentor sichert, sie aber auch auf eine bestimmte Art und Weise gefangen hält (Screenshot Nr. 13). Lediglich eine kleine Klappe in der Mauer neben dem Eingangstor gewährt einen Blick in die Außenwelt. Direkt hinter dem Eingangstor befindet sich ein lebendiger Markt mit verschiedenen Verkaufsständen. Vom Eingangstor zum Haus führt ein Kiesweg, vorbei an gepflegten Blumenbeeten. Der Hinterhof, der in den Sequenzen 51 und 61 auftaucht, ist der einzige, eher ungepflegte Fleck des städtischen Anwesens. In Sequenz 51 verbrennt Noé, der Gärtner, in der Mitte dieses Hinterhofs das zerstörte Klavier der Hausherrin Aída und schaufelt in Sequenz 61 die zurückgebliebene Asche auf einen Haufen. Fausta fühlt sich in diesem Garten sichtlich wohl, die zwitschernden Vögel und Noé, der ihr neue Blumen und Pflanzen zeigt, begeistern sie. Ganz anders geht es ihr im Innern des Anwesens. Die düsteren, einengenden Flure, die wieder an ein Labyrinth erinnern, sind ihr nicht geheuer. Sie scheint deutlich angespannter und ängstlicher zu sein, denn sie schaut sich immer über die Schulter, um zu prüfen, ob ihr jemand folgt. Von draußen scheint kein einziger Lichtstrahl in das Haus, 
die einzige Lichtquelle wird durch Lampen auf Tischen oder an Wänden verströmt. Es ist erkennbar, dass die Einrichtung und Dekoration aus vergangenen Zeiten stammen und sichtlich wertvoll sind, dies gibt Auskunft über die gesellschaftliche Schicht und Zugehörigkeit der Hausbesitzerin. Im Gegensatz zur prunkvollen Gestaltung der Wohnräume von Aída ist das Zimmer, in dem Fausta übernachten soll, sehr spärlich und einfach eingerichtet (Sequenz 18, 23, 24, 40, 47): Ein einfaches Bett mit dunklem Holz, keine Dekoration und das dazugehörende Badezimmer ist mit weißen Fließen bedeckt. Außer dem Bett sind keine weiteren Möbel zu sehen. Es kann angenommen werden, dass die Zuweisung des Zimmers demnach als eine Form der Herabsetzung von Fausta gesehen werden kann.

\section{$\underline{\text { Kostüm und Maske der Figuren }}$}

Enthaltsamkeit bei der Wahl von Kostüm und Maske der Figuren stellt eine Möglichkeit dar, im Film von minimalistischen Mitteln Gebrauch zu machen. Beide sind in La teta asustada besonders minimal und authentisch gehalten. Fausta trägt kein sichtbares Make-up und ist schlicht gekleidet. Sie besitzt wohl nur ein Schmuckstück, das sie aber immer trägt: Eine silberne Kette mit einem Taubenanhänger - das Symbol der Taube taucht noch häufiger auf in diesem Film. Fausta bevorzugt lockere, bequeme Kleidung, die ihre weibliche Figur kaschiert. Auffallend ist dabei, dass sie - bis auf eine Ausnahme (und zwar die Hochzeit ihrer Cousine) - immer eine Hose trägt. Sogar unter der Arbeitsuniform, die sie im Haus von Aída tragen muss, behält sie ihre Hose mit hochgewickelten Hosenbeinen an. Diese Uniform setzt sich zusammen aus einer pastellgrünen Strickjacke und einem Rock, der etwas über das Knie reicht und dessen Farbe vom Licht abhängig ist. Manchmal wirkt er beige und manchmal hellgrün. Dies ist ein beabsichtigter Effekt, dessen Wirkung im nächsten Unterkapitel genauer betrachtet werden soll.

Alle anderen Figuren sind realistisch und unauffällig gekleidet, passend zum jeweiligen Gesellschaftstand und ihrem Beruf angemessen. Das heißt im Alltag wird einfache Kleidung getragen, zu besonderen Anlässen wird sich aber schick gemacht, Handwerker tragen passende Arbeitskleidung und Ärzte einen Ärztekittel. Es gibt einen Kontrast zwischen der Kleidung von Aída oder ihrem Publikum und ihren Angestellten. Unter den Bewohnern von Manchay und der Familie Huamán ist lediglich Máxima etwas auffälliger gekleidet. Sie trägt sehr weibliche und das Dekolleté betonende Kleidungsstücke, außerdem Make-up. Sie wirkt im Gegensatz zu den anderen Bewohnern eher extravagant. Auch ihr Hochzeitskleid ist üppig und hat einen enorm langen Schleier, der mit rosa Luftballons zum Fliegen gebracht 
werden soll - dies scheitert allerdings, was bei Máxima Missmut auslöst. In Sequenz 52 verbringt die Familie den Tag am selbstgebauten Wasserloch (Screenshot Nr. 14). Máxima sticht mit ihrer pinken Bademontur samt Sonnenhut, ebenfalls farblich hervor. In Manchay ist gelegentlich die ein oder andere traditionelle Tracht aus den Anden auszumachen.

\section{$\underline{\text { Symbolik }}$}

In Claudia Llosas Film sind Symbole allgegenwärtig und formen einen wichtigen Bestandteil, um abstrakte Ideen darzustellen oder nicht Erklärbares mit einem speziellen Bild sichtbar zu machen. Oft sind symbolische Aussagen jedoch nicht leicht zu entschlüsseln und zu deuten. Zu Beginn soll sich auf das Bild der Taube konzentriert werden. Tauben sind fester Bestandteil der Hochzeiten, die die Familie Huamán plant und begleitet. Sie werden in einem Käfig auf dem Dach ihres Zuhauses auf nicht artgerechte Weise gehalten. Fausta ist für die Verpflegung der Tauben zuständig. Zum Einsatz kommen sie dann als Überraschung, wenn sie aus der Hochzeitstorte flattern. In Sequenz 13 entwischen sie der Tante, bei dem Versuch sie in die Torte zu stecken. Sie versuchen zu fliehen und schwirren im Wohnzimmer an der Decke entlang. Selbst wenn die Tauben nicht sichtbar im Bild sind, sind sie oft akustisch wahrnehmbar. Ihr Gurren gehören zu den alltäglichen Hintergrundgeräuschen in Manchay. In Sequenz 11 bastelt Fausta eine Papiertaube aus dem Rezept ihres Arztes und lässt sie in eine Schüssel Wasser fallen. Außerdem trägt sie durchgehend eine silberne Kette mit einem Taubenanhänger. Fausta kann sich in einer gewissen Art und Weise mit den Tauben identifizieren. Beide befinden sich in Gefangenschaft und wollen dieser entkommen. Die Taube symbolisiert demnach die Freiheit und die Leichtigkeit, die Fausta für sich und ihre Mutter ersehnt.

Warum sich Fausta eine Kartoffel in die Vagina einführt, wurde bereits erwähnt. Was sich jedoch Claudia Llosa bei diesem Objekt gedacht hat, gilt es jetzt aufzuzeigen. Ein erster Gedanke war, dass die Kartoffel eines der wichtigsten Grundnahrungsmittel nicht nur in Peru, sondern in ganz Südamerika, darstellt. Bei den Inkas wurde die Kartoffel als Sinnbild für Fruchtbarkeit gesehen. Diese Bedeutung scheint sie auch heutzutage noch zu tragen. Bestätigt wird dieses Bild unter anderem durch die Tradition, der Máxima an ihrer Verlobungsfeier nachgeht. Sie schält in Sequenz 36 vor den Augen der Gäste eine überdurchschnittlich große Kartoffel. Eine Überlieferung der indigenen Gesellschaft besagt, dass die Länge der Schale Auskunft über die Beschaffenheit der zukünftigen Ehe gibt. Je 
länger die abgeschnittene Schale, desto glücklicher und Gewinn bringender wird die Ehe der Braut. Die Tatsache, dass Fausta eine Kartoffel benutzt, um ihre Fruchtbarkeit zu unterdrücken, ist paradox. Wie alle Pflanzen, braucht auch eine Kartoffelpflanze Erde, um zu gedeihen. Sie schlägt jedoch Wurzeln im Körper von Fausta, was bedeutet, dass Fausta einen fruchtbaren Nährboden für die Kartoffel verkörpert. Auf der anderen Seite ist sie ein Parasit, der Fausta alle Kräfte raubt und sie krank macht. Die Infektion bereitet ihr große Schmerzen und verursacht außerdem Ohnmachtsanfälle. Fausta kürzt regelmäßig die Triebe der Kartoffel und hindert sie dadurch, sich zu entfalten. Umgekehrt gilt dies genauso: Die Kartoffel hindert Fausta an ihrer eigenen persönlichen und körperlichen Entfaltung. Erst, als Fausta die wichtige Entscheidung trifft, sie aus ihrem Innern entfernen zu lassen, können beide - die Kartoffel und Fausta - sich entfalten. Der Gärtner Noé macht Fausta am Ende des Films ein Geschenk. Es handelt sich um eine blühende Kartoffelpflanze (Screenshot Nr. 15/ 16). Fausta hat sich zu diesem Zeitpunkt bereits von ihrer Mutter und den mit ihr verbundenen Erinnerungen verabschieden können.

Die ersehnte Erlösung finden Fausta und ihre Mutter beim Anblick des Meeres an der Pazifikküste (Screenshot Nr. 17). Der Pazifische Ozean und seine Küste spielen für das Volk der Quechua eine bedeutende Rolle. Sie symbolisieren den Ursprung des Volkes der Quechua: "[L]a costa peruana, lugar de nacimiento del quechua, del dios protector de los comerciantes, Pachacámac" (Bernales Albites/ Gómez 2017: 101). Auf der Reise zum Heimatort der Mutter entschließt sich Fausta gegen die Bestattung des mumifizierten Leichnams in der Erde der Anden. „Fausta se niega a enterrar a su madre en la sierra, quebrantando el deseo de los antiguos dioses precristianos, porque al atravesar por el umbral del dolor supera el trauma y encuentra un nuevo y, a su vez, antiguo dios, Pachacámac" (Bernales Albites/ Gómez 2017: 103). Diese spontane Handlung spiegelt Faustas neugewonnene Unabhängigkeit und Stärke wieder. Die Loslösung von der Mutter ist notwendig, um als eigenständiges Individuum existieren zu können. Dies beinhaltet ebenfalls die Möglichkeit eine andere Person lieben zu können und körperlichen Kontakt mit ihr zu pflegen. Diese Möglichkeit wird gegen Ende des Films mit Noés liebevoller Geste angedeutet.

\section{Musik, Geräusche und Stille}

Im Film verbinden sich die visuelle und die auditive Ebene zu einer Einheit. „Erst der Ton (>sonic space<) verhilft dem bewegten Bild zu einer räumlichen Illusion” (Faulstich 2002: 
90). Beim Ton im Film muss zwischen drei Ebenen unterschieden werden: Zum einen die Geräusche, zum anderen die Musik und zum dritten die Sprache (vgl. Faulstich 2002). Hintergrundgeräusche erzeugen im Film eine ,akustische Atmosphäre, die den Wirklichkeitseindruck des Visuellen wesentlich steigert“" (vgl. ebd.). Da es in La teta asustada nur lakonische Dialoge und stumme Interaktion gibt, dominieren die anderen beiden Ebenen, Geräusche und Musik. Trotzdem hat Claudia Llosa weitestgehend auf den Einsatz von Soundeffekten zur Verstärkung des Wirklichkeitseindrucks verzichtet. Die Hintergrundgeräusche sind aufs Minimum reduziert. So kommt es, dass es in manchen Sequenzen komplett still ist. Allgemein dominieren in internationalen Kinos immer noch Filmproduktionen aus den USA. Daher ist das Publikum vom Kino eher abrupt und laut einsetzende Geräusche sowie gefühlsverstärkende Musik gewohnt. Aus diesem Grund wirkt der Sound in La teta asustada sehr natürlich bis minimalistisch, ja sogar befreit von unnötigem akustischen Überfluss. Geräusche dienen hier nicht zur Emotionalisierung des Zuschauers, auch nicht um Spannung aufzubauen. Es handelt sich lediglich um Geräusche, die Teil der dargestellten Realität sind, also authentische handlungsunterstützende Geräusche.

Dank der Geräusche kann man, selbst wenn man die Augen schließt, erkennen, an welchem Ort sich die Figuren befinden. Im Wohnviertel Manchay wird das Bild von Geräuschen wie dem Rauschen des Windes, gelegentlichem Verkehrslärm, Hundebellen, Taubengurren und Kindergeschrei begleitet. Die Geräusche werden äußerst dezent eingesetzt und lenken nicht von der Handlung ab. Ein Geräusch, das Fausta in Schrecken versetzt, dem Zuschauer jedoch zu Beginn kaum auffällt, erscheint in Sequenz 9. Es handelt sich dabei um das Scharren im Sand, das entsteht während der Onkel ein Loch im Innenhof gräbt. Das Loch soll Faustas verstorbenen Mutter als Grab dienen. Faustas Wunsch ist jedoch, die Mutter auf würdevolle Weise in ihrem Heimatdorf in den Anden zu begraben. Daher erschrickt sie bei diesem Geräusch.

In der Stadt sind die Geräusche etwas lauter, jedoch kommen sie auch hier dezent zum Einsatz. Im Garten des städtischen Anwesens ist nichts von den Geräuschen der Stadt zu hören, sie werden unterdrückt durch die hohen Steinmauern. Dort dominieren Naturgeräusche: Vogelgezwitscher, zirpende Grillen, das Wiehern eines Pferdes - welches jedoch nie gezeigt wird. Diese Geräusche unterstützen das Bild des idyllischen Gartenparadieses.

Was jedoch der unterschwelligen Emotionalisierung der Filmhandlung dient, ist die 
Filmmusik. Sie kann „Erregung verstärken, Stimmung verdichten, ein Lebensgefühl veranschaulichen [...] oder als Requisit zur Spannungssteigerung dienen“ (Faulstich 2002: 139).

Musik im Tonfilm fällt in zwei Kategorien: Source Music und Underscore, oder diegetische und extradiegetische (auch non-diegetische) Musik. Diegetische Musik existiert in der ,Welt' des Films und ist unmittelbar im dramatischen Geschehen verankert (Hetschel/ Moormann 2018: 1).

Die diegetische Filmmusik ist ein Teil der Handlung und hat dadurch auch Einfluss auf die Erzählung, übernimmt also eine narrative Funktion. Dabei ist meistens erkennbar, woher die Musik kommt und inwiefern sie Teil der Handlung ist. Die Quelle kann dabei ein Primärmedium sein, z.B. in Form eines Sängers/einer Sängerin oder einer (Live-)Band. Die Musik kann aber auch von einem Sekundärmedium abgespielt werden, z.B. von einem Radio, Fernsehen oder Smartphone. Sobald die Tonquelle im Bild zu sehen ist, ist die Rede von On-Musik oder synchroner Musik, andernfalls spricht man von Musik im Off oder asynchroner Musik.

Extradiegetische Musik existiert außerhalb der Welt des Films und dient als Kommentar oder emotionelles Manipulationsmittel (ebd.). Dabei handelt es sich um Musik, die speziell für einen bestimmten Film komponiert wurde oder aus bereits existierender Musik zusammengesetzt wurde. Sie wird generell häufiger genutzt, da sie das Geschehen prägt aber selten vom Zuschauer bewusst wahrgenommen wird (vgl. Faulstich 2002).

Nicht jedoch in La teta asustada. Bei genauer Betrachtung fällt auf, dass nur in ausgewählten, meist aufeinanderfolgenden Sequenzen eine extradiegetische Musik, zur Untermauerung der Handlung eingesetzt wird. Diese extradiegetische Musik verdeutlicht das Konzept des Minimalismus im Film. In den Sequenzen 6, 8, 11, 12, 45, 46, 58, 59, 76, 77, 78 und 84 spielt im Hintergrund eine sanfte, aber trauererfüllte Instrumentalmusik. Sie ist sehr unaufgeregt, allerdings sehr emotionsträchtig. Filmmusik ist vor allem dann sehr wirksam, wenn sie sich im Hintergrund hält. Was genau ist an dieser Musik aber minimalistisch? Zum einen handelt es sich um eine einzige Gitarre, die gespielt wird und zum anderen sind es zum größten Teil lediglich einzelne Akkorde, die erklingen. Meistens überlappt die Gitarrenmusik aufeinanderfolgende Sequenzen und verbindet dadurch jene, die thematisch miteinander übereinstimmen und Handlungen fortsetzen. Anders formuliert, die Musik schafft Kontinuität zwischen den Bildern. Nur einmal, zwischen Sequenz 6 und 8, wird die Musik erst unterbrochen und setzt dann später wieder ein. Die Gitarrenmusik taucht immer in Verbindung mit Faustas Anwesenheit auf. Des Weiteren wurde die Musik 
genrespezifisch und unter Berücksichtigung der ethnischen Musiktraditionen sorgfältig ausgewählt.

In den Sequenzen 31 und 35 erklingt ein meditativer, sphärischer Klang, welcher entweder durch Glocken oder das Instrument Hang erzeugt wurde. In beiden Sequenzen befindetsich Fausta im Garten des Anwesens in Lima. Ebenfalls sieht der Zuschauer in beiden Sequenzen das zerstörte Klavier auf dem Kiesweg im Garten und die zerstreuten, bunten Glasscherben. Musik und Gesang gehören zu den aussagekräftigsten Gestaltungsmitteln in La teta asustada. In diesem Fall ist die diegetische Filmmusik, also die Musik, die tatsächlich Gegenstand der Handlung ist, von besonderer Aussagekraft. Vor allem der Gesang ist in diesem Film von zentraler Bedeutung. Er übernimmt die Funktion, die ursprünglich Dialoge haben, also Kommunikation, Vermittlung von Informationen und mentalen Zuständen. Fausta hat Schwierigkeiten mit ihren Mitmenschen zu kommunizieren, darum wählt sie den Gesang, um ihr Inneres, also Gedanken, Ängste und Gefühle zum Ausdruck zu bringen. „Fausta encuentra consuelo recitando canciones que le enseñó su madre desde pequeña porque "quien canta, su mal espanta"” (web. Galván Reyes; 30.08.2018).

Singing is central to conveying Fausta`s inner state throughout the film, as she sings what she cannot say. The importance of song is immediately highlighted since the film`s beginning. The opening scene is framed around song (Vilchez 2014: 10).

Gleich in den ersten beiden Sequenzen des Films erzählt Perpetua, erst aus dem Off dann aus dem On, ihre schicksalsreiche Vergangenheit in einem Lied, das sie voller Schmerz singt:

Quizás algún día/ Tú sepas comprender/ Lo que lloré/ Lo que imploré de rodillas/ A esos hijos de perra/ Era de noche gritaba/ Los cerros remedaban/ Y la gente reía/ Con mi dolor luché diciendo/ A ti te habrá parido/ Una perra con rabia.../ Por eso le has comido tú/ Sus senos/ Ahora pues, trágame a mí/ Ahora pues, chúpame a mí/ Como a tu madre/ A esa mujer que les canta/ Que de noche le agarraron/ Le violaron/ No les dio pena/ De mi hija no nacida/ No les dio vergüenza/ Esta noche me agarraron, me violaron/ No les dio pena que mi hija/ Les viera desde dentro/ Y no contentos con eso/ Me han hecho tragar/ El pene muerto/ De mi marido Josefo/ Su pobre pene muerto sazonado/ Con pólvora/ Con ese dolor gritaba/ Mejor mátame/ Y entiérrame con mi Josefo (La teta asustada 2009: 0.43- 4.18 min).

Sie liegt im Sterben und singt im Beisein ihrer einzigen Tochter Fausta ein letztes Mal von ihrem Leiden. Fausta hört zu, pflegt sie und antwortet, ebenfalls singend: „Cada vez que te acuerdas cuando lloras mamá ensucias tu cama con lágrimas de pena y sudor/ No has comido nada/ Si no quieres comer sólo dímelo y no preparo nada" (ebd.). Perpetua fährt fort: "Comeré si me cantas y riegas esta memoria que se seca/ No veo mis recuerdos, es como si ya no viviera” (ebd.). Während Fausta das Bett richtet - „A ver siéntate bien/ estás tirada como un 
pájaro muerto/ Voy a arreglar la cama un poquito" (ebd.) - stirbt Perpetua und man hört, am Ende der zweiten Sequenz, einige Sekunden lang nur das Rauschen des Windes, bevor es ganz still wird. Fausta singt auch wenn sie allein ist, um sich in Stresssituationen zu beruhigen. Nachdem der Onkel herausgefunden hat, was Fausta ihrem Körper angetan hat, um sich zu schützen, kann sie dies nicht wirklich in Worte fassen. Darum singt sie ihrer toten Mutter auf Quechua vor, um ihre Gründe zu erklären und sich zu rechtfertigen:

El tío no me entiende, ma/ Yo llevo esto como protector/ Yo lo vi todo desde tu vientre/ Lo que te hicieron, sentí tu desgarro/ Por eso ahora llevo esto/ Como un escudo de guerra/ Como un tapón/ Porque sólo el asco/ Detiene a los asquerosos" (La teta asustada 2009: 13.1414.01).

Als Aída sie dann gewissermaßen zwingt zu singen, bedeutet dies für Fausta einen Eingriff in die Intimsphäre und es kostet sie viel Überwindung, denn der Gesang stellt für sie den Ausdruck ihres Schmerzes dar, der durch die im Mutterleib erlebte Gewalt ausgelöst wurde. Doch das Lockmittel mit dem Aída sie ködert, kann ihr die Beerdigung der Mutter ermöglichen. Das Lied, dessen Melodie Aída letztendlich kopiert und als ihre eigene Komposition ausgibt, heißt La sirena. Die Melodie wurde von Selma Catherine Mutal komponiert.

Dicen en mi pueblo que los músicos/ hacen un contrato con una sirena/ Si quieren saber cuánto durará/ durará el contrato con esa sirena/ De un campo oscuro tienen que coger/ un puñado de quinua para la sirena/ Ya si la sirena sigue de contando/ dice la sirena que cada grano significa un año/ Cuando la sirena termine de contar/ se lo lleva al hombre y le suelta al mar/ Pero mi madre dice, dice, dice que la quinua difícil de contar es y la sirena se cansa de contar/ Ya es el hombre para siempre, ya se queda con él don (La teta asustada 2009: 53.37- 55.17).

Trotz der herabsetzenden Beziehung zwischen Aída und Fausta, kann der Moment der Ankunft in dieser neuen Umgebung als Beginn des Befreiungsprozesses gesehen werden. Erst singt Fausta zögerlich, mit leiser, wackliger Stimme. Ihre Stimme wird aber immer kräftiger und stärker, ihr Selbstvertrauen wächst mit jedem Lied und jeder Perle. Der Erfolg des Klavierkonzerts, oder vielmehr, der Erfolg ihrer eigens komponierten Melodie, entlockt ihr in Sequenz 68 darüber hinaus ein Lächeln (Screenshot Nr. 18).

\section{$\underline{\text { Kameraführung }}$}

Die meist verwendeten Einstellungen in La teta asustada sind extreme close-up, close-up, close shot, medium long shot und medium shot. Die Wiederholung bestimmter Einstellungsgrößen wird als Maßnahme des minimalistischen Filmstils betrachtet. Mit der 
Einstellung extreme close-up wird auf bestimmte Körperteile fokussiert. Dieses Verfahren wird Parzellierung genannt.

[Die Parzellierung ist] die Reduktion der ganzheitlichen Erscheinung des Schauspieler auf spezifische Teile des Körpers und auf spezielle Qualitäten im Filmbild. Die Kamera, auf die hin der Schauspieler pointiert spielt, verlangt oft nur Aus- und Anschnitte, mimische und gestische Details, während der Rest des Körpers für diese Aufnahme nicht gebraucht wird. Die Großeinstellung beispielsweise interessiert sich nur für den mimischen Ausdruck, nicht für den ganzen Schauspieler, sie verstärkt und vergrößert den mimischen Ausdruck [...] Der Schauspieler muss unter wechselnden Rahmenbedingungen seinen ganzheitlich verstandenen Körperausdruck auf die Produktion spezifischer Bewegungsdetails reduzieren und seine gesamte Intensität in die Gestaltung dieser Details konzentrieren (Hickethier 2007: 162/163; Anm. der Verfasserin).

Durch diese Hervorhebung von Extremitäten - durch den Bildrand abgetrennte Arme, Füße, Hände oder Gesichter - wird an die Zeit des Terrorismus in Peru erinnert. In dieser Zeit wurden im Departement Ayacucho viele Menschen von Terroristen gefoltert. Die Darstellung von spezifischen Teilen des Körpers kann demnach als eine Anspielung an die zahlreichen verstümmelten Opfer des Terrorismus gesehen werden.

In Sequenz 4 (Screenshot Nr. 19/20), als Fausta im Krankenhaus aufwacht und von einer Krankenschwester auf etwas herablassende Art befragt wird, liegt sie in der Horizontalen im Gynäkologenstuhl. Von der Decke scheint ihr das grelle Neonlicht ins Gesicht, was nur zum Teil in einer extreme close-up Einstellung gezeigt wird, der Rest ihres Gesichts ist vom Bildrand abgeschnitten. Kurz danach, auch in einer extremen Großaufnahme sieht man ihre in die Höhe gestreckten Beine, ebenfalls vom Bildrand abgeschnitten. In Sequenz 7 befinden sich Fausta und Lúcido im Bus auf dem Nachhauseweg, außerdem mehrere vom unteren Bildrand abgetrennte Arme (Screenshot Nr. 21), die man nicht ihren Besitzern zuordnen kann. Auch in Sequenz 9 kann lediglich Faustas Profil im extreme close-up gesehen werden. Des Weiteren sieht man - immer im extreme close-up gedreht - die Hand der Tante, die eine Hochzeitstorte verziert in Sequenz 13; in Sequenz 23 Faustas Füße, zwischen denen die abgeschnittene Wurzel der Kartoffel liegt; Faustas Auge und Nase durch das kleine Kontrollfenster in der Steinwand und von der anderen Seite der Mauer Noés Hand, die er an das Gitter des Fensters hält in Sequenz 35; Sequenz 36, darin schält Máxima eine Kartoffel - eine Tradition während der Verlobungsfeier - und diese Schale fällt ihr zwischen die vom Bildrand abgetrennten Füße; in Sequenz 46 Perpetuas Haare während Fausta sie glatt über das Kopfkissen streicht; in Sequenz 55 wieder Faustas Füße und die Wurzel in ihrer Mitte; in Sequenz 60 sehen eine rote Blume in Faustas Mund; in Sequenz 67 die Waage mit den Perlen und Faustas Zeigefinger, mit dem sie die Perlen zählt, die sie sich schon verdient hat; 
Sequenz 79 zeigt die Perlen in Faustas Hand; in der letzten Sequenz (86) riecht Fausta an der Kartoffelpflanze, die Noé ihr vor das Tor gestellt hat und lächelt.

Die Art und Weise der Kameraführung in La teta asustada ist in den meisten Sequenzen besonders geruhsam. Bis auf wenige Ausnahmen gibt es generell keine hektischen Kamerabewegungen. Bei diesen Ausnahmen handelt es sich um Sequenzen, in denen die Zuschauer in die Perspektive von Fausta hineinversetzt werden. Diese Methode wird subjektive Kamera genannt. Es ist eine Kameraeinstellung, welche dem Zuschauer „die Sicht eines Protagonisten aufzwingt und damit [dessen] Parteilichkeit stimuliert“ (Faulstich 2002: 120, Anm. der Verfasserin). Dadurch wird die Handlung aus der Sicht des Darstellers oder der Darstellerin gezeigt. Die Zuschauer befinden sich in den selben Räumen wie Fausta, folgen ihr auf Schritt und Tritt und bekommen dadurch das Gefühl, als würden sie das Geschehen aus ihren eigenen Augen sehen. Die subjektive Kamera wird jedoch keinesfalls durchgehend im Film eingesetzt. Diese kurzzeitige subjektive Filmperspektive kommt also im Wechsel mit der objektiven Kamera vor. In den folgenden Sequenzen wird die subjektive Kameraeinstellung angewendet: 6, 9, 11, 15, 16, 25, 30, 35, 36, 38, 41, 42, 45, 52, 58, 70, 77, 82, 83. Entweder filmt die Kamerafrau Natasha Brier direkt über Faustas Schulter oder sie nimmt eine Position ein, die Fausta seitlich leicht versetzt begleitet und filmt. Die subjektive Kamera taucht daher häufig in Verbindung mit der Vorwegfahrt oder der Hinterherfahrt der Kamera auf, was ab und an ein Wackeln des Bildes zur Folge haben. Dies bringt jedoch wieder etwas Hektik in das Geschehen. Die subjektive Kamera ist ein stilbildendes Element, das verschiedene Funktionen übernimmt, die im nächsten Unterkapitel genauer beschrieben werden.

Ein Aspekt der Montage, der an dieser Stelle angesprochen werden sollte, ist die mehrmals auftretende Schwarzblende. Zwischen den Sequenzen 23 und 24 gibt es drei Sekunden lang die erste Schwarzblende. Kurz davor, also in Sequenz 23, schneidet Fausta eine Wurzel der sprießenden Kartoffel ab, die auf den Boden zwischen ihre Füße fällt. In der Sequenz nach der Schwarzblende, sitzt sie regungslos auf dem Bett im Zimmer, das ihr in Aídas Anwesen zugeteilt wurde. Die nächste Schwarzblende erfolgt zwischen den Sequenzen 55 und 56 ebenfalls drei Sekunden lang und die Schwarzblende wird abermals eingesetzt nachdem sich Fausta eine Wurzel abschneidet. Zwischen den Sequenzen 70 und 71 dauert die Schwarzblende vier Sekunden. Diese wird eingeblendet nachdem Aída ihre nach dem Konzert nutzlose Angestellte aus dem Auto wirft und diese in der stockdunklen Nacht an der Straße entlang rennt und nach Hause finden soll. Die letzte Schwarzblende erscheint 
zwischen den Sequenzen 84 und 85. Diesmal dauert sie sogar 11 Sekunden lang und wird von der bereits bekannten Gitarrenmusik hinterlegt. Vor dieser Schwarzblende trägt Fausta ihre mumifizierte Mutter auf dem Rücken über hohe Sanddünen zum Meeresufer, sie singt auf Quechua und verabschiedet sich so von ihrer geliebten Mutter.

Farben und Licht

„Farben spielen in Filmen eine ganz wichtige Rolle - als Stilmittel atmosphärischer Gestaltung, als Handlungshinweise oder auch als symbolische, die Message indizierende Bedeutungsträger“" (Faulstich 2002: 147). Farbe ist ein Gestaltungsmittel, das in diesem Film stark reduziert, jedoch äußerst symbolträchtig eingesetzt wird. Dies bedeutet nicht, dass es ein farbloser Film ist, sondern, dass Farben meist Ton in Ton auftauchen und diese starke Bedeutungen haben. Diese Art von Einfarbigkeit nennt man auch Monochromie, was als ein Element des Minimalismus auf visueller Ebene gilt und in La teta asustada Anwendung findet. Die Farben im Film sind an die gezeigten Orte gebunden. Farben, die immer wiederkehren, sind Naturtöne wie Steingrau, Sandfarben, Beige, Braun, außerdem verschiedene Nuancen von Blau und Grün, Rosa und Orange. Eine Szene im Stadtviertel Manchay beispielsweise ist farblich immer ähnlich aufgebaut. Dort dominieren die Farben Beige, Sandfarben, Braun, Grau und Himmelblau. Der Garten in Aídas Anwesen dagegen mischt endlos viele Nuancen von saftigem Grün. Im Inneren des Herrenhauses erkenntman warme, dunkle Farben, wie Orange, Dunkelbraun und Dunkelgrün. Eine Farbe, die jedoch ortsübergreifend sehr häufig auftaucht, ist ein pastelliges, eher ins grün gehendes Türkis.

Trotz teilweise ungünstigen Lichtverhältnissen, unter anderem in Manchay oder im Herrenhaus, wurde nicht zu starken, künstlichen Leuchtmitteln gegriffen, sondern das natürliche Tages- bzw. Sonnenlicht genutzt. Dadurch wirkt der Film oft etwas monochrom, vor allem bei starkem Wolkengang.

\section{Erzielte Wirkung und Effekte}

Ziel des Minimalismus als Filmstil ist es, die Geschwindigkeit aus der Handlung zu nehmen und den Fokus auf das alltägliche Leben zu setzen. Diese Entschleunigung der Handlung hat ebenso eine Verstärkung der inneren Zustände der Figuren im Sinn. Die Minimalisierung der Gestaltungsmittel bewirkt daher die Betonung von Faustas Entwicklung und ihrer Gefühlswelt. Aufgrund des minimalistischen Stils gibt es keine 
Ablenkung durch zu schnelle Handlungsabläufe, zu viele Ortswechsel, zu laute und irritierende Nebengeräusche, zu viele Figuren, extravagante Kostüme oder unrealistisch schöne Schauspieler. All das trifft auf den Film La teta asustada zu. Er ist ein Figurenfilm, der von einer jungen Frau handelt, der es gelingt ihre Ängste zu überwinden und beginnt zu leben. Der Zuschauer verfolgt die persönliche Entwicklung von Fausta und der Bewältigung ihres Traumas. Bezogen auf die Geschichte Perus, kann man Fausta auch als Träger einer kollektiven Erinnerung sehen, denn die Folgen dieses bewaffneten Konfliktes sind immer noch sichtbar. Diese Schäden gilt es zu reparieren und zu überwinden, um weiterzuleben. Im vorherigen Unterkapitel wurden die Gestaltungsmittel beschrieben, die minimalisiert wurden, um die Wahrnehmung des Zuschauers auf die innere Befreiung der Protagonistin zu lenken. Hier wird nun erklärt, welche Wirkung und Effekte dies auf den Zuschauer und dessen Rezeption des Films hat. Die oben genutzte Reihenfolge der minimalisierten Elemente wird auch hier Anwendung finden, um deren Wirkung zu beschreiben. Zuerst wird die Wirkung der Verringerung der Anzahl von Sequenzen und des Ortswechsels erörtert, später folgen die erzielten Effekte der Minimalisierung von Kostümen und Maske, sowie von Musik und Nebengeräuschen. Ebenso wird die Anwendung von Symbolen diskutiert. Die minimalisierten Gestaltungselemente in der Kameraführung und die naturalistische Anwendung von Farben und Lichtverhältnissen wird ebenfalls Thema sein.

Die Verringerung der Anzahl von Sequenzen, das heißt deren zeitliche Verlängerung ist wohl die offensichtlichste Methode, um den Film zu entschleunigen. Das Bild wird verlangsamt, die Augen des Zuschauers werden nicht überanstrengt, man sieht die Handlungen der Figuren in ihrer Gänze. Die Zusammenhänge der Handlungen sind deutlich dargestellt. Darum müssen die Zuschauer nicht zu viel Konzentration aufbringen, um dem Handlungsablauf folgen zu können. Es gibt genügend Zeit, um Fausta sowie ihre Mimik und Gestik zu beobachten und zu analysieren. Durch diese Entschleunigung des Geschehens, kommt Claudia Llosa der Darstellung des realen Alltagslebens sehr nah.

Ebenfalls die Verringerung der Ortswechsel vereinfacht die Handlung. In La teta asustada wird nicht auf Quantität, sondern auf die Qualität der Drehorte geachtet. Claudia Llosa und ihr Team haben sehr stark auf die symbolische Darstellung der Orte geachtet. Da der Zuschauer sich meist auf die filmischen Charaktere und deren Geschichten konzentriert, wird der filmische Raum oft nur als narrativer Raum wahrgenommen. Nichtsdestotrotz hat 
dieser im Film, wie auch im richtigen Leben, eine symbolische Bedeutung. Dies resultiert vor allem aus dem Vergleich mit anderen Räumen und stellt eine der wichtigsten Bedeutungsebenen dar (vgl. Mikos 2008: 115). Anhand der Darstellung des ruralen und urbanen Raums können auch die Emotionen der handelnden Figuren dargestellt werden. Der Film kann so auch, wie in zahlreichen Raumtheorien festgehalten, Veränderungen des ruralen und urbanen Raums in der Zeit und des menschlichen Verhaltens seiner Bewohner festhalten. Viele Darstellungen des Raums führen daher zu einem Diskurs über soziale Probleme, wobei sich die Räume oft als Freund oder Feind der Protagonisten präsentieren. In La teta asustada sind die Rollen, die die verschiedenen Räume einnehmen, klar gekennzeichnet. Für Fausta ist Manchay der Raum, in dem sie und ihre Krankheit - von dem einen zwar mehr als von anderen - akzeptiert werden. Dort muss sie sich nicht verstellen, denn Faustas soziale Ängste sind bekannt und werden respektiert. Die Stadt jedoch fürchtet sie, dort ist sie sichtlich verkrampft und angespannt. Doch nach und nach versteht sie, dass sie diese Hürden überwinden muss, um ihr Ziel zu erreichen und nimmt die Hindernisse daher auf sich. Sie arbeitet und übernachtet in einer fremden Umgebung, freundet sich mit einem unbekannten Mann an und singt, um das Begräbnis ihrer Mutter zu finanzieren.

Die Darstellung der zwei Orte, Manchay und Lima, ist eine Anspielung auf die gesellschaftliche Teilung in Peru, ein Land, in dem manche Menschen im Überfluss leben und andere wiederum in Armut und Ungleichheit. So werden zwei verschiedene Welten oder Realitäten gezeigt, die unterschiedlicher nicht sein könnten. Die Mauer um Aídas Anwesen symbolisiert die Barriere zwischen diesen Welten. Minimalismus spielt bei der Darstellung dieser Räume eine wichtige Rolle. Der Minimalismus bei der Komposition der Bilder in Manchay spiegelt die Realität wieder, ist deshalb eine Form, um den Raum naturalistisch zu gestalten. Was die verschiedenen Drehorte verbindet, ist das Motiv des Labyrinths. Das Motiv des Labyrinths wird nicht nur auf der visuellen Ebene angewendet. Die Tatsache, dass immer wieder dieselben Drehorte gezeigt werden, nimmt es ebenfalls auf einer höheren, den gesamten Film strukturierenden Ebene auf. Während Faustas Befreiungsprozesses kreuzt das Labyrinth wiederholt ihren Weg: im Wohnviertel Manchay, auf dem Weg über den gut besuchten Markt in Lima, auch in Aídas Anwesen. Es ist ein Symbol für die Suche nach dem Ausweg, nach einer Lösung, die Fausta von ihren Phobien befreit. Wie bereits oben erwähnt, soll das Labyrinth auch den Alltag ohne Veränderung darstellen. Fausta ist wie gefangen in ihrer Situation und vergeudet ihre Jugend, in dem sie in der Vergangenheit und von ihren Ängsten gelenkt, lebt. In ihrem Leben gibt es wenig Freude, ab und zu entwischt ihr ein 
Lächeln - meist, wenn sie Kinder sieht - doch sonst ist sie wie versteinert und die Angst ist ihr ins Gesicht geschrieben.

Das Leiden, das Fausta quält, stammt aus der Zeit in den Anden, die unter den Bewohnern des Gebirges als Sasachacuy oder el gran miedo bekannt sind (vgl. Theidon 2004). Von diesem Leiden befreit sich Fausta in der Stadt, dem urbanen Raum, in dem sie Gefahren überwinden muss. Diese städtische Erfahrung, bestehend aus ihrer ersten Arbeit und dem Kontakt mit Aída und Noé, gibt ihr Selbstvertrauen und stellt einen Lernprozess dar. Das Trauma, welches durch Fausta verkörpert wird, ist mit der gesellschaftlichen und geografischen Isolation in Manchay verknüpft, in der sie lebt. Erst als sie diese Isolation durchbricht, indem sie sich in ein neues Umfeld wagt, beginnt sie sich von dem Trauma zu erholen. Dort wird sie nicht mit ihrer Mutter und deren schrecklichen Erfahrungen konfrontiert. Das Eindringen in den urbanen Raum bricht demnach auch mit ihren vergangenen und sozialen Einschränkungen, die sie sich selbst aufgebürdet hat. Dieser Befreiungsprozess äußert sich vor allem in ihrer Entscheidung, sich das zu nehmen, was ihr rechtmäßig zusteht und wieder Herrin ihres eigenen Körpers zu sein und sich nicht von Schmerzen, verursacht durch einen Fremdkörperin ihr, kontrollieren zu lassen (vgl. Salinas 2016). Die vorletzten Sequenzen 83 und 84 zeigen die endgültige Befreiung ihres Traumas. Der Anblick des Meeres weckt in ihr die Erinnerung an den Satz der zweiten Sargverkäuferin in Sequenz 12 als Fausta sich den Sarg genauer anschaut, auf dem ein Strand und das Meer gemalt sind: „Ese te traiga la paz. Es el mar del Pacífico dónde se alivian sus cargas ylavan sus penas“ (La teta asustada 2009: 16.29- 18.27) und an Aídas enttäuschte Aussage, als sie die Puppe aus ihrer Kindheit in der Erde wiederfindet: „Me dijeron que si la enterraba, luego la tierra se la llevaba y nunca la encontrabas [kurze Pause] mentirosos“" (La teta asustada 2009: 59.29- 1.00.58). Sie hat das Bedürfnis, sich selbst und ihre Mutter von den traumatischen Erfahrungen in den Anden zu lösen und reinzuwaschen. Fausta entscheidet sich somit, die Mutter nicht wieder an den Ort zu bringen, an dem all die seelischen und körperlichen Schmerzen begonnen haben, sondern an einen Ort, an dem sie tatsächlich Ruhe und Frieden finden kann. Zusammen mit der physischen Last, dem mumifizierten Körper der Mutter, begrabt Fausta ihre seelischen Lasten am Ufer des Pazifiks. Die Darstellung der weiten, endlosen Sandlandschaft und der Blick auf den Ozean, der sich bis zum Horizont erstreckt, fallen zusammen mit der Entlastung der Protagonistin.

Die Kostüme und Maske der Figuren im Film sind sehr natürlich gehalten. Fausta wird durch 
die Farbgestaltung ihrer Kleidung, die sie auf der Arbeit trägt, äußerlich an ihre Umgebung angepasst. Gelegentlich wirkt es, als läge ein blaugrüner Filter über der Kamera, der den Garten auf Aídas Anwesen zusammen mit Fausta farblich aufeinander abstimmt. Sie wirkt durch diesen Lichteffekt wie ein Chamäleon, das sich farblich seiner Umgebung anpassen und dadurch von anderen nicht gesehen werden kann. Es kann angenommen werden, dass Aída genau das mit der Wahl der Arbeitsuniform bezweckt. Sie verrichtet leise ihre Arbeit und wirkt dabei wie ein Geist, der durch die labyrinthartigen Flure im Herrenhaus schleicht. Die langen Haare, die Fausta sonst offen trägt, um ihr Gesicht zu verstecken, muss sie auf dem Anwesen zu einem Zopf binden, was dazu führt, dass sie ihren Kopf immer etwas nach unten neigt und auf den Boden schaut, um Blickkontakt mit Anderen zu vermeiden. Dies strahlt ebenfalls eine untergebenen Haltung aus und passt zu ihrer unterwürfigen Rolle als Dienstmädchen.

Ein Symbol ist an sich kein minimalistisches Element, denn hinter diesem Symbol stehen Bedeutungen und Sachverhalte, die nicht mit einfachen Worten oder dargestellten Handlungen gezeigt werden können. Was jedoch ein minimalistisches Element bildet, ist die Wiederholung von Symbolen. Dies ist der Fall im Film La teta asustada. Tauben, Kartoffeln und der Pazifische Ozean sind Symbole die darin immer wieder auftreten und die Traumatisierung, beziehungsweise die Befreiung des Traumas thematisieren.

Durch die subjektive Kameraeinstellung wird der Zuschauer direkt in das Geschehen hineinversetzt. Er bekommt das Gefühl direkt beteiligt zu sein und die Handlung aus den Augen von Fausta zu sehen. Ziel dieser speziellen Kameraeinstellung ist es, Faustas Empfindungen intensiver für den Zuschauer zu gestalten. Wenn sie beispielsweise verängstigt über den lauten, überfüllten Markt in Lima läuft und die Kamera ihr hinterher eilt, bewirkt die subjektive Einstellung dieselbe Anspannung beim Zuschauer, die Fausta empfindet. Die subjektive Kamera, vor allem in Verbindung mit der Vorweg- oder Hinterherfahrt, intensiviert dagegen die dargestellte Handlung und vermittelt mehr Authentizität. Die subjektive Kamera kann daher auch als ein Gestaltungsmittel des realistischen Kinos gesehen werden. Der Wechsel zwischen subjektiver und objektiver Kamera vermittelt mehr Spannung, denn so bekommen die Zuschauer ebenfalls Bilder aus einer beobachtenden Position zu Gesicht. Dies schafft eine gewisse Distanz zwischen den Figuren der Handlung und den Zuschauern. Dadurch werden vor allem sehr traurige und dramatische Szenen wieder aufgelockert. Unter anderem ist dies der Fall in Sequenz 70, in 
der Fausta nach dem Konzert aus Aídas Auto geworfen wird. Der Zuschauer bleibt jedoch im Innern des Autos sitzen und lässt Fausta allein in der Dunkelheit, was ein Schuldgefühl in ihm auslöst (Screenshot Nr. 22). Bis auf wenige Ausnahmen, bewahrt die Kamera eine ruhige Position. Dadurch werden die Spannungskurven flach gehalten. Es wird nur eine geringe emotionale Steigerung beim Zuschauer ausgelöst.

Schwarzblenden werden in diesem Film genutzt, um zu zeigen, dass es Sachverhalte gibt, für die weder Worte noch Bilder passend erscheinen. Der minimalistische Filmstil präferiert eine vielsagende Schwarzblende. Es ist eine Leerstelle, die gleichzeitig eine beispiellose Aussagekraft in sich trägt.

Farben und Lichtverhältnisse werden in La teta asustada in geringemMaße verwendet. Die Wiederholung der Farbe Türkis ist Ausdruck des Minimalismus. Türkis ist eine Farbe, die eigentlich eher selten in der Natur vorkommt und darum umso wertvoller erscheint, falls sie doch natürlicherweise auftritt. Es ist eine Übergangsfarbe zwischen Blau und Grün. Mit Türkis wird der Himmel, das Meer und klares Wasser, das in der Sonne glitzert, verbunden. Aufgrund dieser Assoziationen ist Türkis eine Farbe die Wohlbefinden, Entspannung und Ausgeglichenheit vermittelt. In der Esoterik hat die Farbe Türkis ebenfalls eine besondere Wirkung auf Körper und Geist. Sie soll für einen ruhigen, erholsamen Schlaf sorgen, gegen Entzündungen und psychische Störungen helfen, das Immunsystem stärken und Kraft spenden. Die Effekte der Farbe Türkis nach Feng Shui sollen ebenfalls sehr positiv sein. In Wohnräumen sorgt sie für Harmonie und eine entspannende Atmosphäre. Der Edelstein Türkis kann wohl neues Selbstbewusstsein schenken und Infektionen lindern.

Es ist wahrscheinlich kein Zufall, dass diese Farbe gewählt wurde. All diese positiven Effekte der Farbe Türkis würden Fausta von ihren Leiden befreien und ihr neuen Lebensmut schenken. Die Wirkung auf den Zuschauer ist ebenfalls nicht zu unterschätzen. Trotz der tragischen Geschichte, den unbehaglichen Situationen, in denen sich Fausta befindet und ihrer sichtlich bedrückenden Gestik und Mimik, kann eine positive Grundhaltung und die Hoffnung auf ein Happy End beibehalten werden.

Die naturalistische Lichtgestaltung trägt viel zur Glaubwürdigkeit des Gezeigten im Film bei. Genauso wie die Farbgestaltung ist auch das Licht ein dramaturgisches Element, das die Wahrnehmung oder Rezeption des Zuschauers stark beeinflusst. Dieser naturalistische Beleuchtungsstil schafft eine authentische, natürliche und realistische Stimmung. Im Herrenhaus allerdings wurde eine Low-Key-Beleuchtung genutzt, das ist ein 
Beleuchtungsstil, bei dem die Dunkelheit überwiegt und die Sequenz dadurch als düster, unheimlich, fast schon beängstigend empfunden wird. Eventuell wurden die Lichtverhältnisse der Sequenzen, die im Innern des Anwesens gefilmt wurden, in der Postproduktion noch am Computer verändert, um sie noch dunkler zu gestalten. Der Angstzustand, dem Fausta ausgesetzt ist, soll damit dem Publikum besser vermittelt werden. Der Zuschauer bekommt somit ebenfalls das Gefühl, dass sich in den Gängen womöglich etwas Gefährliches befinden könnte.

\section{Abschließender Kommentar und Fazit}

In diesem Kapitel werden die Ergebnisse der durchgeführten Analyse des Films $\mathrm{La}$ teta asustada, der dem Genre Cinema of Difficult Dialogues zugeordnet werden kann, zusammengefasst. Der Film stellt das Alltagsleben einer modernen, bilingualen IndioFamilie in der peruanischen Post-Konflikt-Gesellschaft nach.

Als Grundlage wurden im zweiten Kapitel zunächst die Filmdramaturgie und die Traumaforschung beschrieben und anschließend zueinander in Beziehung gesetzt. Für das Verständnis des Films und dessen Analyse war dies relevant, da er die Minimalisierung der filmischen Gestaltungsmittel nutzt, um über ein intergenerationelles Trauma aufzuklären. Der heutige Stand der Forschung, die sich mit dem intergenerationellen Trauma befasst, ist nach wie vor auf die Traumatisierung begrenzt, die durch den Holocaust provoziert wurde. Die Ergebnisse dieser Forschung können jedoch auf weitere intergenerationelle Traumata, wie beispielsweise la teta asustada, übertragen werden. Grund der Übertragung einer Traumatisierung an nachfolgende Generationen ist unter anderem das Stillschweigen, das die Opfer bewahren, sobald es um ihre grausamen Erfahrungen geht. Außerdem führt das Fehlen einer angemessenen Traumaverarbeitung sowie einem Schuldeingeständnis der Täter zum Fortleben des Traumas im (Unter-)Bewusstsein der Nachkommenschaft der Opfer. Posttraumatischer Stress vergeht daher nicht mit dem Ableben der direkten Betroffenen, sondern bleibt im Innern deren (Enkel-)Kinder fortbestehen.

Im zweiten Kapitel wurden außerdem wichtige dramaturgische Begriffe erklärt. Die Betonung lag dabei auf der Definition des Minimalismus im Film. Minimalistische Prinzipien im Film beabsichtigen, mithilfe von stark reduzierten künstlerischen und technischen Mitteln, das Alltagsleben der Figuren für den Zuschauer intensiv erfahrbar zu 
machen, was jedoch nicht bedeutet, dass alle Gestaltungsmittel auf ihr Minimum verringert werden müssen.

In Kapitel drei wurde über die Terrororganisation Sendero Luminoso und deren strukturelle Tötungsdelikte informiert. Sie wird als die für die Opfer hauptverantwortliche Partei des bewaffneten Konfliktes in Peru gesehen. Im Jahr 2003 veröffentlicht die Comisión de la Verdad y Reconciliación (CVR) peruana ihren Informe final. Dieser Aufklärungsbericht beweist, dass in den letzten beiden Jahrzehnten des 20. Jahrhunderts an die 69,280 Menschen ermordet wurden. $79 \%$ der Opfer lebten in ländlichen Gebieten und 75\% waren quechuasprachige Bewohner der Anden. Im selben Kapitel wurde außerdem Theidons Aufklärungsstudie vorgestellt, deren Ziel es ist, die Veränderungen der sozialen und politischen Strukturen zu untersuchen, die noch heute die ethnische Diskriminierung, Ausgrenzung und Unruhe verstärken. Theidon interviewte die Bewohner der Region Ayacucho, die vom bewaffneten Konflikt betroffen waren. Diese Interviews enthalten Informationen über verschiedene Krankheitsbilder, die in der Allgemeinmedizin unter dem Begriff PTBS zusammengefasst werden können. Die Krankheiten werden jedoch von den Betroffenen selbst sehr mystisch und bildlich dargestellt. Die meist psychischen Leiden werden körperlich sehr stark wahrgenommen. Das heißt, sie beschrieben körperliche Beschwerden, die jedoch nicht auf konkrete organische Befunde oder bewiesene körperliche Fehlfunktionen zurückzuführen waren. La teta asustada, eines dieser Leiden, ist die intergenerationelle Traumatisierung, an denen Kinder von vergewaltigten Frauen leiden, die den susto in sich tragen. Um diese Traumatisierung dreht sich die Handlung des gleichnamigen Films.

Ziel der Arbeit war es, zu zeigen, dass sich der innere Befreiungsprozess der Protagonistin Fausta von übertragenen traumatischen Erinnerungen und Ängsten im minimalistischen Einsatz der filmischen Bauformen wiederspielt. Im vierten Kapitel, der Analyse, wurde die Darstellung dieses intergenerationellen Traumas untersucht. Um die Traumatisierung und die Genesung von Fausta so intensiv wie nur möglich darzustellen, wurde der minimalistische Filmstil angewandt. Der Minimalismus manifestiert sich im Film auf der narrativen, diegetischen, visuellen und auditiven Ebene sowie bei der Inszenierung der Darsteller. Im Film La teta asustada angewandte Elemente des Minimalismus sind die Folgenden: Echtzeitdarstellungen, lange Erzähldauer in einzelnen Sequenzen, Schilderung von Alltagsleben, flache Spannungskurven, Reduzierung der Anzahl von Figuren und Nebendarstellern, eingeschränkte Ausstattung mit wenig Dekor, wenig Kostümvariationen, 
Nutzung von realen Schauplätzen, Verminderung von Reizen, Monochromie, reduzierte Belichtung, Wiederholung von bevorzugten Einstellungsgrößen, statische Kamera, lakonische Dialoge, sparsame akustische Modulierungen, kleine Gesten und sparsame Gesichtsausdrücke.

In Anbetracht der vorher präsentierten Ergebnisse, lässt sich zusammenfassen, dass die im Film angewandten minimalistischen Gestaltungsmittel dazu dienen, eine realistische, naturalistische Atmosphäre zu schaffen. La teta asustada kreiert eine realistische Wirklichkeitsabbildung der Bewohner im Wohnviertel Manchay in der Nähe von Lima. Die Aufnahmen wurden an Originalschauplätzen gemacht, was dem Zuschauer das Geschehen realistischer erscheinen lässt. Der Film interpretiert die Alltagswirklichkeit von Nachkommen der Opfer des bewaffneten Konfliktes in den 1980ern und 1990ern. Genauer genommen interpretiert er das Leben der jungen Frau Fausta, die an der, innerhalb des Volkes der Quechua weit verbreiteten, Krankheit namens la teta asustada leidet. Llosas Film stellt demnach Menschen mit Schicksalen dar, die in diesen gesellschaftlichen und kulturellen Kreisen alltäglich sind. Der Film basiert auf wahren Begebenheiten, die in der Studie von Theidon erörtert wurden und mischt dabei reale Elemente mit fiktiven Bestandteilen. Der Film legt den Fokus auf den Bewältigungsprozess, den Fausta mit wenig äußerlicher Hilfe meistert. Die reduzierten Bauformen lenken davon nicht ab.

Auf künstlerische Art und Weise hilft der Film über die Geschehnisse der heutigen Gegenwart in Peru aufzuklären. Ebenfalls übt er Kritik an bereits tief verwurzelten gesellschaftlichen Strukturen, die die Diskriminierung von ethnischen Minderheiten fördern. Daher kann La teta asustada auch als Ausdruck des Verlangens nach Gleichberechtigung, Gleichstellung und Chancengleichheit der quechuasprachigen Bevölkerung des Landes verstanden werden. Da ein konkretes Schuldeingeständnis der Täter und die Hilfe für die Rekonstruktion der Andendörfer und der Familien ausblieb, ist der Film auch ein Ausdruck des Verlangens nach einer angemessenen reconciliación. In dieser Arbeit wurde hervorgehoben, dass es nötig ist, auch in künstlerischen Formen soziale Probleme und Ungerechtigkeiten anzusprechen. Filme wie La teta asustada sind unabdingbar, um Menschen, die politische Gewalt erfahren mussten und von der Regierung nicht entschädigt oder therapeutisch betreut wurden, eine Stimme und eine Möglichkeit zu geben, sich von der Traumatisierung zu lösen. Ebenfalls sollen Filme wie dieser beim Publikum nicht nur Sympathie und Mitleid für die Figuren wecken, sondern auch Solidarität mit Überlebenden eines gewaltsamen Konfliktes. 


\section{Bibliographie}

\subsection{Literatur}

BARROW, Sarah. „Out of the Shadows: 'New' Peruvian Cinema, National Identity and Political Violence“. Modern Languages Open. Liverpool University Press (2014): 117.

BARSAM, Richard M. und Dave MONAHAN. Looking at movies : an introduction to film. 3. ed. New York: Norton, 2010.

BECKER, Wolfgang und Norbert SCHÖLL. Methoden und Praxis der Filmanalyse: Untersuchungen zum Spielfilm und seinen Interpretationen. Opladen: Leske Budrich, 1983.

BEDOYA, Ricardo. „Nuestro cine: Una historia intermitente“. Libros y Artes, 2002, $14-17$.

BERNALES ALBITES, Enrique und Leila GOMEZ. „Trauma y aislamiento en La teta asustada de Claudia Llosa; Trauma and Isolation in Claudia Llosa's The Milk of Sorrow“. IBEROAMERICANA Vol. 17, Nr. 65 (2017): 93-106.

CHAUCA, Edward. „Mental Illness in Peruvian Narratives of Violence after the Truth and Reconciliation Commission." Latin American Research Review. Vol. 51, Nr. 2 (2016): 67-85.

CHAUCA, Edward M., Rafael RAMÍREZ und Carolina SITNISKY. „,,No pretendo retratar la realidad. Pretendo interpretar un tema para sacar discusiones que tenemos reprimidas': Una entrevista con Claudia Llosa“. Mester Vol. 39 (2010): 45-55.

ESPINOZA, Damarys. „Superando la teta asustada: Structural violence, intergenerational trauma, and indigenous Peruvian women's agency“, NACCS Annual Conference Proceedings, Nr. 5 (2010).

FAULSTICH, Werner. Grundkurs Filmanalyse. München: Fink, 2002.

GROB, Norbert, Bernd KIEFER, Roman MAUER und Josef RAUSCHER. Kino des Minimalismus. 1. Aufl. Bd. 3. Genres \& Stile. Mainz: Bender, 2009.

HENTSCHEL, Frank. Filmmusik: ein alternatives Kompendium. Herausgegeben von Peter Moormann. Wiesbaden: Springer VS, 2018.

HICKETHIER, Knut. Film- und Fernsehanalyse. 4., aktualis. und erw. Aufl. Stuttgart/ Weimar: Metzler, 2007.

IOSA, Emilio, Tomás IOSA, Marcela LUCCHESE, María Soledad BURRONE, Rubén ALVARADO, Eliecer VALENCIA und Ruth FERNÁNDEZ. „Transmisión transgeneracional del trauma psicosocial en comunidades indígenas de Argentina: 
percepción del daño en el pasado y presente y acciones autoreparatorias“. Cadernos Saúde Coletiva 21, Nr. 1 (2013): 85-91.

ISMAR, Georg und Rosmery CUEVA SÁENZ. „Eine ,Mauer der Schande“ zerteilt Lima“. n-tv.de, 29. Oktober 2017. https://www.n-tv.de/panorama/Eine-Mauer-der-Schandezerteilt-Lima-article20107457.html.

KIM, Tae-Hwan. Vom Aktantenmodell zur Semiotik der Leidenschaften: eine Studie zur narrativen Semiotik von Algirdas J. Greimas. Tübingen: Narr, 2002.

KLOTZ, Volker. Geschlossene und offene Form im Drama. München: Hanser, 2012.

KÖHNE, Julia Barbara und Thomas BALLHAUSEN. Trauma und Film: Inszenierungen eines Nicht-Repräsentierbaren. Berlin: Kulturverlag Kadmos, 2012.

KORTE, Helmut. Einführung in die systematische Filmanalyse: ein Arbeitsbuch; mit Beispielanalysen. 4., Neu bearb. und erw. Aufl. Berlin: Schmidt, 2010.

LAMPARTNER, Ulrich, Christa HOLSTEIN, Linde APEL, Malte THIESSEN, Dorothee WIELING, Birgit MÖLLER und Silke WIEGAND-GREFE. „Die familiäre Weitergabe von Kriegserfahrungen als Gegenstand interdisziplinärer Forschung“, ZPPM Zeitschrift für Psychotraumatologie, Psychotherapiewissenschaft, Psychologische Medizin, 8 Jahrgang, Heft 1 (2010): 9-23.

LAURA ATANACIO, Vanessa Liz. „Feminidades filmadas: Madeinusa (2006) y La Teta Asustada (2009) entre la tradición y la transgresión.“ Pontificia Universidad Católica del Perú, 2016.

MASEDA, Rebeca. „Indigenous Trauma in Mainstream Peru in Claudia Llosa’s The Milk of Sorrow.“ Hispanic Journal of Theory and Criticism Vol. 6 (11) (2016).

MHANDO, Martin und Keyan G. TOMASELLI. „Film and trauma: Africa speaks to Itself through truth and reconciliation films“. Black Camera: An International Film Journal (The New Series) 1, Nr. 1 (2009): 30-50.

MIKOS, Lothar. Film- und Fernsehanalyse. 2., überarb. Aufl. Konstanz: UVKVerl.-Ges., 2008.

MOTTE, Warren. Small Worlds: Minimalism in Contemporary French Literature. Stages : 13. Lincoln, 1999.

PRAMAGGIORE, Maria und Tom WALLIS. Film: a critical introduction. London: King, 2005.

RABENALT, Peter. Filmdramaturgie. 1. Aufl., überarb. Ausg. d. Erstausg. Berlin/ Köln: Alexander, 2011. 
SALINAS, Pablo. „El mar en la representación cinematográfica de la migración interna en el Perú: de Gregorio a La teta asustada“. Confluencia Vol. 31, Nr. 2 (2016): 11327.

SÁNCHEZ VILLAGÓMEZ, Marté Eulogio. „El horror olvidado. Memoria e historia de la violencia política en Ayacucho, Perú (1980-2000)“. Universitat Autònoma de Barcelona, 2015.

STUCCHI-PORTOCARRERO, Santiago und Vanessa HERRERA-LOPEZ. „Cine peruano actual y psicopatología / Current Peruvian cinema and psychopathology“. Rev Neuropsiquiatr 77 (4) (2014): 207-13.

STUTTERHEIM, Kerstin und Silke KAISER. Handbuch der Filmdramaturgie: das Bauchgefühl und seine Ursachen. 2., überarb. und erw. Aufl. Frankfurt am Main/ Berlin/ Bern/ Wien [u.a.]: Lang, 2011.

THEIDON, Kimberly. Entre prójimos- el conflicto armado interno y la política de reconciliación en el Perú. Estudios de la Sociedad Rural 24. Lima: IEP Ediciones, 2004.

VILCHEZ, Jennifer Ann. „The Body, the Abject and Horror in Claudia Llosa's La teta asustada“. Central European University, 2014.

YEHUDA, Rachel und Linda M. BIERER. „Transgenerational transmission of cortisol and PTSD risk“. In Progress in Brain Research, herausgegeben von E. Ronald De Kloet, Melly S. Oitzl und Eric Vermetten, 167:121-35. Stress Hormones and Post Traumatic Stress Disorder Basic Studies and Clinical Perspectives. Elsevier, 2007. https://doi.org/10.1016/S0079-6123(07)67009-5.

\subsection{Internetquellen}

ANONYM. manchayperu. „Nosotros“. manchayperu (blog), 16. November 2016. https://manchayperu.com/2016/11/16/nosotros/.

NEDOMANSKY, Vashi. „Average Shot Length Archives - Blog“, 23. August 2018. http://vashivisuals.com/the-average-shot-length-of-david-fincher-films/.

NIKOLAIDIS, Leo. „The Magic Realism of Peruvian Claudia Llosa's 'La Teta Asustada' and 'Madeinusa'“. Sounds and Colours, 4. September 2010. https://soundsandcolours.com/articles/peru/the-magic-realism-of-peruvian-claudia-llosasla-teta-asustada-and-madeinusa-1813/.

GALVÁN REYES, Mario. „Un viaje al miedo de la libertad: La teta asustada, lo mejor del nuevo cine latinoamericano“, 29. August 2018. http://icarorevista.weebly.com/lateta-asustada.htm 
Journal of Science,

Humanities and Arts

July 2019

Volume 6, Issue 7 


\section{Anhang}

Hier im Anfang befindet sich das Sequenzprotokoll, das für die Analyse von La teta asustada erstellt wurde. Des Weiteren werden Screenshots von beschriebenen Sequenzen angehängt.

1.1 Sequenzprotokoll

\begin{tabular}{|c|c|c|c|c|c|c|c|c|c|}
\hline Nr. & $\begin{array}{l}\text { Zeit } \\
(\min )\end{array}$ & Ort & $\begin{array}{l}\text { Figuren } \\
\text { (Kostüm und } \\
\text { Maske) } \\
\end{array}$ & Handlung & Symbole & $\begin{array}{l}\text { Musik/ } \\
\text { Geräusche/ } \\
\text { Stille } \\
\end{array}$ & $\begin{array}{l}\text { Kameraführung } \\
\text { (Einstellungsgröße, - } \\
\text { perspektive, } \\
\text { Bewegungen) }\end{array}$ & $\begin{array}{l}\text { Farben und } \\
\text { Licht }\end{array}$ & Mise-en-scène \\
\hline 1 & $\begin{array}{l}0.43- \\
2.05\end{array}$ & $\begin{array}{l}\text { BLACK } \\
\text { SCREEN }\end{array}$ & BLACK SCREEN & $\begin{array}{l}\text { Off: Perpetua } \\
\text { erzähltsingendihr } \\
\text { Schicksal }\end{array}$ & & $\begin{array}{l}\text { Off: Gesang } \\
\text { Perpetua, Wind }\end{array}$ & BLACK SCREEN & BLACK SCREEN & BLACK SCREEN \\
\hline 2 & $\begin{array}{l}2.06- \\
4.18\end{array}$ & A. & $\begin{array}{l}\text { Perpetua und } \\
\text { Fausta* } \\
\text { ohne Make-up } \\
\text { offene Haare } \\
\text { einfache Kleidung, } \\
\text { Fträgt(imganzen } \\
\text { Film) eine silberne } \\
\text { Kette mit } \\
\text { Taubenanhänger }\end{array}$ & $\begin{array}{l}\text { Perpetua liegt im } \\
\text { Bett, erzählt } \\
\text { singend ihre } \\
\text { Geschichte (mit } \\
\text { geschlossenen } \\
\text { Augen, Fpflegt sie } \\
\text { und ordnet ihr } \\
\text { Bett (singend), } \\
\text { Perpetua stirbt }\end{array}$ & $\begin{array}{l}\text { Labyrinth } \\
\text { Taubenkette }\end{array}$ & $\begin{array}{l}\text { On: Gesang } \\
\text { Perpetua, F } \\
\text { Gesang, } \\
\text { am Ende der } \\
\text { Szene: Wind } \\
\text { und dann } \\
\text { komplette Stille }\end{array}$ & $\begin{array}{l}\text { Aufblende } \\
\text { 1. close-up, Zoom } \\
\text { zurück (Perpetua) } \\
\text { F bewegt sich in } \\
\text { das Bild } \\
\text { 2. close shot, Zoom } \\
\text { nach vorne (F) } \\
\text { normal camera } \\
\text { height }\end{array}$ & $\begin{array}{l}\text { versch. } \\
\text { Türkistöne, } \\
\text { rosa/ } \\
\text { Tageslicht vom } \\
\text { Fenster } \\
\text { (rechts), F } \\
\text { bewegt sich } \\
\text { vor das } \\
\text { Fenster, } \\
\text { Gegenlicht } \\
\text { (Gesicht im } \\
\text { Schatten) }\end{array}$ & $\begin{array}{l}\text { altes, schäbiges } \\
\text { Bettgestell, } \\
\text { kitschige Tapete } \\
\text { mitRosen, beides } \\
\text { bröseltbereitsab, } \\
\text { Sicht aus dem } \\
\text { Fenster: ärmliches } \\
\text { Andendorf, keine } \\
\text { Grenzen zu sehen, } \\
\text { wirkt wie ein } \\
\text { Labyrinth ohne } \\
\text { Ausgang }\end{array}$ \\
\hline \multicolumn{8}{|c|}{ TITEL EINBLENDUNG } & $\begin{array}{l}\text { rot, gelb, grün, } \\
\text { türkis }\end{array}$ & \\
\hline 3 & $\begin{array}{l}4.32- \\
6.49\end{array}$ & B. & $\begin{array}{l}\text { Tío, Tante, } \\
\text { Máxima, andere } \\
\text { Familien- } \\
\text { mitglieder und } \\
\text { Kinder, F }\end{array}$ & $\begin{array}{l}\text { Familiewidmet } \\
\text { sich versch. } \\
\text { Aktivitäten, } \\
\text { Máxima will einen } \\
\text { noch längeren }\end{array}$ & & $\begin{array}{l}\text { Wind, Scharren } \\
\text { im Sand, Tiere, } \\
\text { Straße (Hupen, } \\
\text { Autos) }\end{array}$ & $\begin{array}{l}\text { 1. medium long } \\
\text { shot (Familie), } \\
\text { kaum } \\
\text { wahrzunehmender } \\
\text { Zoom nach vorne }\end{array}$ & $\begin{array}{l}\text { türkis, lila, } \\
\text { braun// } \\
\text { mit Wolken } \\
\text { bedeckter } \\
\text { Himmel, Sonne }\end{array}$ & $\begin{array}{l}\text { einfaches } \\
\text { Steinhaus mit } \\
\text { Dach aus Holz und } \\
\text { Planen, } \\
\text { Plastiktisch und }\end{array}$ \\
\hline
\end{tabular}




\begin{tabular}{|c|c|c|c|c|c|c|c|c|}
\hline & & & $\begin{array}{l}\text { einfache Kleidung, } \\
\text { Máxima in } \\
\text { Brautkleid, sehr } \\
\text { langem Schleier }\end{array}$ & $\begin{array}{l}\text { Schleier, Fnähert } \\
\text { sich, siebekommt } \\
\text { Nasenbluten und } \\
\text { wird ohnmächtig }\end{array}$ & & $\begin{array}{l}\text { 2. mediumshot(F), } \\
\text { Kamera statisch, F } \\
\text { bewegt sich auf } \\
\text { Kamera zu bisclose } \\
\text { shot } \\
\text { normal camera } \\
\text { height }\end{array}$ & $\begin{array}{l}\text { hinter Wolken } \\
\text { (v. links) }\end{array}$ & $\begin{array}{l}\text { Stühle, bunte } \\
\text { Möbel, Berge im } \\
\text { Hintergrund }\end{array}$ \\
\hline 4 & $\begin{array}{l}6.50- \\
7.40\end{array}$ & $\begin{array}{l}\text { Kranken- } \\
\text { haus Lima, } \\
\text { Behand- } \\
\text { lungs- } \\
\text { zimmer }\end{array}$ & $\begin{array}{l}\text { F, Kranken- } \\
\text { schwester } \\
\text { ohne Make-up, F } \\
\text { hat blutunter- } \\
\text { laufende Augen } \\
\text { und glänzende, } \\
\text { von der Sonne } \\
\text { gerötete Haut } \\
\text { Kranken- } \\
\text { schwester trägt } \\
\text { Schwestern- } \\
\text { uniform }\end{array}$ & $\begin{array}{l}\text { F wacht im } \\
\text { Krankenhaus auf, } \\
\text { sie wird von der } \\
\text { Krankenschwester } \\
\text { befragt }\end{array}$ & $\begin{array}{l}\text { leise Stimmen } \\
\text { aus dem } \\
\text { Kranken- } \\
\text { hausflur, Klicken } \\
\text { der Neonlampe }\end{array}$ & $\begin{array}{l}\text { 1. extreme close-up } \\
\text { (Gesicht F), } \\
\text { extreme high shot } \\
\text { 2. close shot } \\
\text { (Krankenschwester) } \\
\text { / extreme close-up } \\
\text { (Bein F), low shot } \\
\text { 3. extreme close-up } \\
\text { (Gesicht F), } \\
\text { extreme high shot } \\
\text { 4. close-up } \\
\text { (Neonlampe), } \\
\text { extreme low } \\
\text { camera } \\
\text { statische Kamera }\end{array}$ & $\begin{array}{l}\text { braun, } \\
\text { schwarz, weiß/ } \\
\text { künstliches, } \\
\text { grelles } \\
\text { Neonlicht } \\
\text { scheint von } \\
\text { obenauf F } \\
\text { Gesicht }\end{array}$ & $\begin{array}{l}\text { Behandlungs- } \\
\text { zimmer nur } \\
\text { mit dem } \\
\text { Nötigsten: } \\
\text { Gynäkologen- } \\
\text { stuhl, Vorhang, } \\
\text { Ordner, Ventilator }\end{array}$ \\
\hline 5 & $\begin{array}{l}7.41- \\
10.11\end{array}$ & $\begin{array}{l}\text { Kranken- } \\
\text { haus, } \\
\text { Besprech- } \\
\text { ungs- } \\
\text { zimmer }\end{array}$ & $\begin{array}{l}\text { Tío, Arzt } \\
\text { (F hinter dem } \\
\text { Vorhang) } \\
\text { Arzt: Ärztekittel, } \\
\text { tío: einfache aber } \\
\text { ordentliche } \\
\text { Kleider (Hemd } \\
\text { und Hose) }\end{array}$ & $\begin{array}{l}\text { Arzt klärt den } \\
\text { Onkel über F's } \\
\text { Geheimnis auf, der } \\
\text { Onkel erzählt ihm } \\
\text { von ihrem Leiden, } \\
\text { aber beide reden } \\
\text { aneinander vorbei, } \\
\text { Ffolgt dem } \\
\text { Gespräch hinter } \\
\text { dem Vorhang }\end{array}$ & $\begin{array}{l}\text { Geräusche aus } \\
\text { dem Kranken- } \\
\text { haus, } \\
\text { klingelndes } \\
\text { Telefon }\end{array}$ & $\begin{array}{l}\text { 1. close shot (tío), } \\
\text { Schuss } \\
\text { 2.closeshot(Arzt), } \\
\text { Gegenschuss } \\
\rightarrow \text { mehrmalige } \\
\text { Wdh. } 1+2 \mathrm{im} \\
\text { Wechsel } \\
\text { 3. close-up (F) } \\
\text { statische Kamera } \\
\text { normal camera } \\
\text { height }\end{array}$ & $\begin{array}{l}\text { beige, türkis/ } \\
\text { Tageslicht von } \\
\text { Fenster }\end{array}$ & $\begin{array}{l}\text { einfaches Büro: } \\
\text { Schreibtisch mit } \\
\text { Papieren, } \\
\text { Kommode mit } \\
\text { Infobroschüren, } \\
\text { im Hintergrund } \\
\text { Vorhang zum } \\
\text { Behandlungs- } \\
\text { zimmer }\end{array}$ \\
\hline
\end{tabular}




\begin{tabular}{|c|c|c|c|c|c|c|c|c|c|}
\hline 6 & $\begin{array}{l}10.12- \\
10.56\end{array}$ & $\begin{array}{l}\text { Kranken- } \\
\text { haus, Flur }\end{array}$ & $\begin{array}{l}\text { F, Tío, Patienten } \\
\text { und Krankenhaus- } \\
\text { personal } \\
\text { Alltagskleider } \\
\text { (authentisch) }\end{array}$ & $\begin{array}{l}\mathrm{F} \text { folgt ihrem } \\
\text { Onkel durch den } \\
\text { Krankenhausflur }\end{array}$ & Labyrinth & $\begin{array}{l}\text { Gitarren- } \\
\text { musik }\end{array}$ & $\begin{array}{l}\text { 1. close-up }(\mathrm{F}), \\
\text { Vorwegfahrt } \\
\text { (Kamera vor ihr) } \\
\text { 2. full shot (tío), } \\
\text { subjektive Kamera } \\
\text { (Perspektive von F) } \\
\rightarrow \text { mehrmalige } \\
\text { Wdh. } 1+2 \\
\text { normal camera } \\
\text { height }\end{array}$ & $\begin{array}{l}\text { weiß/ } \\
\text { Tageslicht von } \\
\text { Fenstern, } \\
\text { abwechselnd } \\
\text { Licht und } \\
\text { Schatten }\end{array}$ & $\begin{array}{l}\text { kahler, weißer, } \\
\text { steriler Flur, keine } \\
\text { Sitzmöglichkeiten, } \\
\text { keine Schilder, } \\
\text { Personen sitzen } \\
\text { aufdem Boden an } \\
\text { Wände gelehnt }\end{array}$ \\
\hline 7 & $\begin{array}{l}10.57- \\
12.26\end{array}$ & $\begin{array}{l}\text { Bus (nach } \\
\text { Hause) }\end{array}$ & $\begin{array}{l}\text { F, Tío, Fahrgäste } \\
\text { (Männer) } \\
\text { Altagskleider, alle } \\
\text { tragen relativ } \\
\text { ähnliche Farbtöne }\end{array}$ & $\begin{array}{l}\text { F und Onkel } \\
\text { fahren im Bus } \\
\text { nach Hause, F } \\
\text { erklärt ihm ihre } \\
\text { Gründe }\end{array}$ & Körperteile & $\begin{array}{l}\text { Motorgeräusche } \\
\text { vom Bus, } \\
\text { Stimmen von } \\
\text { Fahrgästen }\end{array}$ & $\begin{array}{l}\text { 1. close-up (F, tío, } \\
\text { Arme und } \\
\text { Gesichter von } \\
\text { anderen), statische } \\
\text { Kamera, } \\
\text { normal camera } \\
\text { height }\end{array}$ & $\begin{array}{l}\text { beige, weiß/ } \\
\text { natürliches } \\
\text { Licht }\end{array}$ & $\begin{array}{l}\text { voller Bus, } \\
\text { Haltestangen, } \\
\text { Köpfe und Arme } \\
\text { von Passagieren }\end{array}$ \\
\hline 8 & $\begin{array}{l}12.27- \\
13.13\end{array}$ & $\begin{array}{l}\text { Fußweg nach } \\
\text { Hause } \\
\text { (Manchay) }\end{array}$ & $\begin{array}{l}\text { F, Tío, Bewohner } \\
\text { Frau am } \\
\text { Straßenrand mit } \\
\text { Strohhut und Rock } \\
\text { (traditionell) }\end{array}$ & $\begin{array}{l}\mathrm{F} \text { folgt dem Onkel } \\
\text { durch das } \\
\text { Armenviertel } \\
\text { Manchay }\end{array}$ & Labyrinth & $\begin{array}{l}\text { Gitarren- } \\
\text { musik, vorbei- } \\
\text { fahrende Autos, } \\
\text { Rufe }\end{array}$ & $\begin{array}{l}\text { 1. extreme long } \\
\text { shot } \\
\text { 2. extreme long } \\
\text { shot } \\
\text { 3. extreme long } \\
\text { shot } \\
\rightarrow 3 \text { versch. } \\
\text { Kamerapositionen, } \\
\text { statische Kamera }\end{array}$ & $\begin{array}{l}\text { sandfarben/ } \\
\text { natürliches } \\
\text { Licht }\end{array}$ & $\begin{array}{l}\text { Landschaft: Sand, } \\
\text { Berge, Stein-/ } \\
\text { Holzhäuser } \\
\text { (Labyrinth) }\end{array}$ \\
\hline 9 & $\begin{array}{l}13.14- \\
14.01\end{array}$ & A. & $\begin{array}{l}\text { F, Leichnam der } \\
\text { Mutter } \\
\text { hellblaue Decke } \\
\text { als Leichentuch }\end{array}$ & $\begin{array}{l}\text { F liegt neben dem } \\
\text { Leichnamihrer } \\
\text { Mutter, sie } \\
\text { vergräbt ihr } \\
\text { Gesicht unterder } \\
\text { Decke, um näher } \\
\text { bei ihrer Mutter zu } \\
\text { sein, sie hört den } \\
\text { Onkel graben }\end{array}$ & & $\begin{array}{l}\text { Off: Gesang F, } \\
\text { Gurren der } \\
\text { Tauben, Graben } \\
\text { im Sand }\end{array}$ & $\begin{array}{l}\text { 1. close-up } \\
\text { (Wasserbeutel), } \\
\text { extreme low } \\
\text { camera/ subj. } \\
\text { Kamera (Persp. F) } \\
\text { 2. extreme close-up } \\
\text { (F's Profil), normal } \\
\text { camera height }\end{array}$ & $\begin{array}{l}\text { sandfarben, } \\
\text { himmelblau/ } \\
\text { natürliches } \\
\text { Licht }\end{array}$ & $\begin{array}{l}\text { an der Decke } \\
\text { hängen mit } \\
\text { Wasser gefüllte } \\
\text { Plastiktüten (um } \\
\text { Fliegen, die } \\
\text { eventuell durch } \\
\text { den Leichnam } \\
\text { angelockt werden, } \\
\text { zu verscheuchen) }\end{array}$ \\
\hline
\end{tabular}




\begin{tabular}{|c|c|c|c|c|c|c|c|c|c|}
\hline & & & & & & & $\begin{array}{l}\text { 3. close-up (Fund } \\
\text { P), high shot }\end{array}$ & & \\
\hline 10 & $\begin{array}{l}14.02- \\
14.56\end{array}$ & B. & $\begin{array}{l}\text { Tío, F } \\
\text { tío: löchriges } \\
\text { Unterhemd, } \\
\text { Hose }\end{array}$ & $\begin{array}{l}\text { Onkel gräbt eine } \\
\text { Grube im Hof, F } \\
\text { erklärt ihre } \\
\text { Wünsche, sie will } \\
\text { ihre Mutter zurück } \\
\text { in ihr Heimatdorf } \\
\text { bringen }\end{array}$ & & $\begin{array}{l}\text { Graben im Sand, } \\
\text { hallende } \\
\text { Stimmen aus } \\
\text { dem Viertel, } \\
\text { Wind, Gurren } \\
\text { der Tauben }\end{array}$ & $\begin{array}{l}\text { 1. close-up, normal } \\
\text { camera height (tío) } \\
\text { 2. medium long } \\
\text { shot, high shot(tío, } \\
\text { Fkommt ins Bild) } \\
\text { 3. close-up (beide), } \\
\text { low shot }\end{array}$ & $\begin{array}{l}\text { sandfarben/ } \\
\text { natürliches } \\
\text { Licht }\end{array}$ & $\begin{array}{l}\text { Blick aus F } \\
\text { Fenster: Dächer } \\
\text { (Wellblech) und } \\
\text { Steinhäuser } \\
\text { (hauptsächlich } \\
\text { Rohbauten, nicht } \\
\text { fertig gestellt, im } \\
\text { Hof gräbt tío ein } \\
\text { Loch in den Sand }\end{array}$ \\
\hline 11 & $\begin{array}{l}14.57- \\
16.28\end{array}$ & A. & $\begin{array}{l}\text { F } \\
\text { F: Haare im Zopf, } \\
\text { einfache } \\
\text { Alltagskleidung, } \\
\text { ohne Make-up }\end{array}$ & $\begin{array}{l}\text { F sitzt auf dem } \\
\text { Bett undfaltet } \\
\text { singend } \\
\text { Servietten, sie } \\
\text { faltet eine } \\
\text { Papiertaube aus } \\
\text { dem Rezept des } \\
\text { Arztes und lässt } \\
\text { sie insWasser } \\
\text { fallen }\end{array}$ & Papiertaube & $\begin{array}{l}\text { On: Gesang F, } \\
\text { Gitarren-musik, } \\
\text { Wasser-tropfen, } \\
\text { Rascheln des } \\
\text { Papiers }\end{array}$ & $\begin{array}{l}\text { 1. extreme close-up } \\
\text { (tropfendes } \\
\text { Nachthemd, Fuß F } \\
\text { verschwommen) } \\
\text { 2. close-up (Hände, } \\
\text { Servietten), high } \\
\text { shot/subj. Kamera } \\
\text { (Persp. F) } \\
\text { 3. close-up (F), } \\
\text { normal camera } \\
\text { height } \\
\rightarrow \text { Wdh. } 2+3 \\
\text { 4. close-up (Hand, } \\
\text { Papiertaube), high } \\
\text { shot } \\
\text { 5. close-up (F's } \\
\text { Profil), normal } \\
\text { camera height }\end{array}$ & $\begin{array}{l}\text { himmelblau, } \\
\text { weiß/ } \\
\text { natürliches } \\
\text { Licht }\end{array}$ & $\begin{array}{l}\text { frisch } \\
\text { gewaschenes } \\
\text { Nachthemd hängt } \\
\text { tropfend von der } \\
\text { Decke, darunter } \\
\text { eine } \\
\text { Plastikschüssel, } \\
\text { die das Wasser } \\
\text { auffängt, F sitzt } \\
\text { auf dem Bett, das } \\
\text { voller gefalteter } \\
\text { Stoffservietten ist, } \\
\text { Jesus-Kalender an } \\
\text { der Wand }\end{array}$ \\
\hline
\end{tabular}




\begin{tabular}{|c|c|c|c|c|c|c|c|c|c|}
\hline & & & & & & & $\begin{array}{l}\text { 6. Schwenk, close- } \\
\text { up (Papiertaube im } \\
\text { Wasser), high shot }\end{array}$ & & \\
\hline 12 & $\begin{array}{l}16.29- \\
18.27\end{array}$ & $\begin{array}{l}\text { Bestattungs- } \\
\text { institute } \\
1 . \text { Verkaufs- } \\
\text { raum } \\
\text { 2. Artesanías- } \\
\text { Särge } \\
\text { 3. Dach } \\
\text { Manchay? }\end{array}$ & $\begin{array}{l}\text { F, Chicho (Cousin), } \\
\text { Sargverkäufer(in), } \\
\text { Kind } \\
\text { Chicho: } \\
\text { umgedrehte Cap, } \\
\text { Muskelshirt } \\
\text { F: Haare im Zopf } \\
\text { Verkäufer: } \\
\text { ordentliche } \\
\text { Kleidung aber } \\
\text { nichts } \\
\text { Extravagantes } \\
\text { (außerdie hohen } \\
\text { Schuhe der 2. } \\
\text { Verkäuferin) }\end{array}$ & $\begin{array}{l}\text { F und Chicho } \\
\text { schauen sich } \\
\text { versch. Särge an } \\
\text { und informieren } \\
\text { sich über die } \\
\text { Preise, im ersten } \\
\text { Bestattungsinstitut } \\
\text { lächelt sie beim } \\
\text { Anblickdes Kindes }\end{array}$ & $\begin{array}{l}\text { F interessiert } \\
\text { sichfür den } \\
\text { Sarg mit dem } \\
\text { gemalten } \\
\text { Meer, } \\
\text { vendedora: } \\
\text { "Eso es el mar } \\
\text { del Pacífico } \\
\text { dónde se } \\
\text { alivian sus } \\
\text { cargas y lavan } \\
\text { sus penas." }\end{array}$ & $\begin{array}{l}\text { Gitarren- } \\
\text { musik, Kirchen- } \\
\text { glocken, } \\
\text { Folklore-Musik, } \\
\text { Geräusche von } \\
\text { der Straße, } \\
\text { Hundebellen }\end{array}$ & $\begin{array}{l}\text { 1a. close shot } \\
\text { (Kind, ab der } \\
\text { Gürtellinie } \\
\text { abwärts), high shot } \\
\text { 1b. close-up (F), } \\
\text { normal camera } \\
\text { height } \\
\rightarrow \text { Wdh. 1a }+1 b \\
\text { 1c. closeshot } \\
\text { (Verk. 1), normal } \\
\text { camera height } \\
\text { 1d. close-up ( } F \text {, } \\
\text { Chicho), normal } \\
\text { camera height } \\
\rightarrow \text { mehrmalige } \\
\text { Wdh. 1c. }+1 d \text {. } \\
\text { 2a. Kamera } \\
\text { befindetsichschon } \\
\text { im Raum, medium } \\
\text { long shot ( } F, C h \text {, } \\
\text { Verk. 2) } \\
\text { 2b. close-up (F, Ch } \\
\text { im Profil), normal } \\
\text { camera height, } F \\
\text { bewegt sich auf } \\
\text { Kamera zu } \\
\text { 2c. close-up (F v. } \\
\text { hinten), normal } \\
\text { camera height } \\
\text { 2d. medium long } \\
\text { shot ( } F, \text { Ch, Verk.) }\end{array}$ & $\begin{array}{l}\text { himmelblau, } \\
\text { farbenfroh, } \\
\text { sandfarben, } \\
\text { weiß/ } \\
\text { natürliches } \\
\text { Licht }\end{array}$ & $\begin{array}{l}\text { 1. seriöse } \\
\text { Dekoration, } \\
\text { Gemälde (Mona } \\
\text { Lisa) und Kerzen, } \\
\text { Kind spielt mit } \\
\text { Autos auf dem } \\
\text { Boden zwischen } \\
\text { Särgen } \\
\text { 2. großer } \\
\text { Ausstellungsraum } \\
\text { mit glänzendem } \\
\text { Boden, bunte, } \\
\text { farbenfrohe } \\
\text { Artesanías-Särgen } \\
\text { je nach } \\
\text { Geschmack } \\
\text { stehen überall im } \\
\text { Raumundhängen } \\
\text { an Wänden } \\
\text { 3. auf dem Dach } \\
\text { eines Steinhauses, } \\
\text { im Hintergrund } \\
\text { BergeundHäuser }\end{array}$ \\
\hline
\end{tabular}




\begin{tabular}{|c|c|c|c|c|c|c|c|c|c|}
\hline & & & & & & & $\begin{array}{l}\text { 2e. close-up ( } \mathrm{F}, \mathrm{Ch} \text {, } \\
\text { Verk.), normal } \\
\text { camera height } \\
\text { 3a. closeshot }(\mathrm{F} \text {, } \\
\mathrm{CH} \text {. Verk. 3), } \\
\text { normal camera } \\
\text { height }\end{array}$ & & \\
\hline 13 & $\begin{array}{l}18.28- \\
19.15\end{array}$ & $\begin{array}{l}\text { Wohnraum } \\
\text { des Onkels } \\
\text { und seiner } \\
\text { Familie }\end{array}$ & $\begin{array}{l}\text { Tante, Máxima, F } \\
\text { Máxima: zeigt } \\
\text { Dekolleté, trägt } \\
\text { Schmuck, ist } \\
\text { geschminkt }\end{array}$ & $\begin{array}{l}\text { Tante verziert eine } \\
\text { Hochzeitstorte, } \\
\text { Tauben flüchten } \\
\text { aus der Torte, F } \\
\text { beobachtet }\end{array}$ & Tauben & $\begin{array}{l}\text { Musik aus } \\
\text { Radio, Gurren } \\
\text { und Flattern der } \\
\text { Tauben }\end{array}$ & $\begin{array}{l}\text { 1. close shot } \\
\text { (Tante, Máxima), } \\
\text { normal camera } \\
\text { height } \\
\text { 2. extreme close-up } \\
\text { (Hand Tante, } \\
\text { Torte), extreme } \\
\text { high shot } \\
\text { 3. close-up (F), } \\
\text { normal camera } \\
\text { height } \\
\rightarrow \text { Wdh. } 1+3\end{array}$ & $\begin{array}{l}\text { türkis, rosa, } \\
\text { sandfarben, } \\
\text { weiß/ } \\
\text { natürliches } \\
\text { Licht }\end{array}$ & $\begin{array}{l}\text { enger Wohnraum, } \\
\text { vollgestellt, } \\
\text { durcheinander, } \\
\text { Hochzeitstorte in } \\
\text { der Mitte auf } \\
\text { einem Tisch, } \\
\text { Tauben fliegen } \\
\text { durch den Raum }\end{array}$ \\
\hline 14 & $\begin{array}{l}19.16- \\
21.13\end{array}$ & A. & $\begin{array}{l}\text { F, Tante, Máxima, } \\
\text { Frauen, Jonathan } \\
\text { Alltagskleider }\end{array}$ & \begin{tabular}{l|} 
Frauen \\
mumifizieren \\
Perpetua's \\
Leichnam, F \\
nimmt Jobangebot \\
an, um Beerdigung \\
zu ermöglichen, \\
dafür akzeptiert \\
sie sogar allein zu \\
sein
\end{tabular} & Mumie & $\begin{array}{l}\text { Off:Gesang + } \\
\text { Schluchzen } \\
\text { Perpetua, } \\
\text { hallende } \\
\text { Stimmen aus } \\
\text { dem Viertel, } \\
\text { Straßen- } \\
\text { geräusche }\end{array}$ & $\begin{array}{l}\text { 1. close shot(alle), } \\
\text { normal camera } \\
\text { height } \\
\text { 2. close-up (F), } \\
\text { normal camera } \\
\text { height } \\
\rightarrow \text { Wdh. } 1+2 \\
\text { 3. mediumfull } \\
\text { shot, extremehigh } \\
\text { shot }\end{array}$ & $\begin{array}{l}\text { türkis, rosa, } \\
\text { sandfarben, } \\
\text { weiß, } \\
\text { himmelblau/ } \\
\text { natürliches } \\
\text { Licht }\end{array}$ & $\begin{array}{l}\text { Bett mittig, links } \\
\text { und rechts } \\
\text { Fenster, Frauen } \\
\text { sitzen um das Bett } \\
\text { herum }\end{array}$ \\
\hline 15 & $\begin{array}{l}21.14- \\
21.53\end{array}$ & $\begin{array}{l}\text { Weg zur } \\
\text { Arbeit/ Markt } \\
\text { (Lima) }\end{array}$ & $\begin{array}{l}\text { F, Tante, } \\
\text { Marktarbeiter } \\
\text { F: offene Haare }\end{array}$ & $\begin{array}{l}\text { F folgt ihrer Tante } \\
\text { über den } \\
\text { hektischen Markt }\end{array}$ & Labyrinth & $\begin{array}{l}\text { Stimmen- } \\
\text { gewirr, } \\
\text { Marktschreie, } \\
\text { hupende Autos }\end{array}$ & $\begin{array}{l}\text { 1. close-up (F), } \\
\text { Vorwegfahrt } \\
\text { (Kamera vor ihr) } \\
\text { 2. medium shot } \\
\text { (Tante), subj. } \\
\text { Kamera (Persp. F) }\end{array}$ & $\begin{array}{l}\text { beige, blau, } \\
\text { türkis, lila } \\
\text { grün/ } \\
\text { natürliches } \\
\text { Licht, am Ende } \\
\text { Schatten }\end{array}$ & $\begin{array}{l}\text { eng aneinander- } \\
\text { gestellte } \\
\text { Marktständemit } \\
\text { hauptsächlich } \\
\text { Lebensmitteln, } \\
\text { labyrinthartige } \\
\text { schmale Gänge }\end{array}$ \\
\hline
\end{tabular}




\begin{tabular}{|c|c|c|c|c|c|c|c|c|}
\hline & & & & & & $\begin{array}{l}\rightarrow \text { mehrmalige } \\
\text { Wdh. } 1+2 \\
\text { 3. full shot, Kamera } \\
\text { verfolgtbeidevon } \\
\text { der Seite } \\
\text { 4. full shot v. hinten }\end{array}$ & & $\begin{array}{l}\text { ohne Ausgang, } \\
\text { dunkles } \\
\text { Eingangstor im } \\
\text { Schatten }\end{array}$ \\
\hline 16 & $\begin{array}{l}21.54- \\
23.46\end{array}$ & C. & $\begin{array}{l}\text { F, Tante, } \\
\text { Haushälterin } \\
\text { Haushälterin: rosa } \\
\text { Kostüm, rosa } \\
\text { Perlenohrringe }\end{array}$ & $\begin{array}{l}\text { F folgt der } \\
\text { Haushälterin } \\
\text { durch den Garten, } \\
\text { blickt erschrocken } \\
\text { zurückalssich das } \\
\text { Tor automatisch } \\
\text { schließt, diese } \\
\text { erklärt ihr die } \\
\text { Aufgaben, warnt } \\
\text { vor Kriminalität }\end{array}$ & $\begin{array}{l}\text { Türklingel, } \\
\text { Stimmen-- } \\
\text { gewirr, Öffnen } \\
\text { des } \\
\text { Eingangstors, } \\
\text { Vogelge- } \\
\text { zwitscher, } \\
\text { Wiehern eines } \\
\text { Pferdes }\end{array}$ & $\begin{array}{l}\text { 1. fullshot(F,Tante } \\
\text { v. hinten) } \\
\text { 2. close shot (Kopf } \\
\text { der Haush.Im } \\
\text { Fenster), normal } \\
\text { camera height } \\
\text { 3. close shot, } \\
\text { Kamera bewegt } \\
\text { sich mit F mit, } \\
\text { wenn F stehen } \\
\text { bleibt, bleibt die } \\
\text { Kamera stehen), } \\
\text { normal camera } \\
\text { height } \\
\text { 4. close-up (F), } \\
\text { Vorwegfahrt } \\
\text { (Kamera vor ihr) } \\
\text { 5. medium shot } \\
\text { (Haush.), } \\
\text { Hinterherfahrt (F } \\
\text { zum Teil v. hinten) } \\
\text { 6. close-up (F), } \\
\text { Vorwegfahrt } \\
\text { (Kamera vor ihr) } \\
\text { 7. medium shot } \\
\text { (Haush.) / subj. } \\
\text { Kamera (Persp. F) } \\
\text { bis Kamera stehen } \\
\text { bleibt }\end{array}$ & $\begin{array}{l}\text { weiß, braun, } \\
\text { grün, rosa, } \\
\text { sandfarben/ } \\
\text { Tageslicht } \\
\text { (hell) }\end{array}$ & $\begin{array}{l}\text { großes, braunes } \\
\text { Eingangstor (wie } \\
\text { Garage), dahinter } \\
\text { andere Welt: } \\
\text { paradiesischer } \\
\text { Garten, } \\
\text { abgetrennt von } \\
\text { dem Rest von } \\
\text { Lima } \rightarrow \text { wie } \\
\text { goldener Käfig }\end{array}$ \\
\hline
\end{tabular}




\begin{tabular}{|c|c|c|c|c|c|c|c|c|c|}
\hline & & & & & & & $\begin{array}{l}\text { 8. medium long } \\
\text { shot (beide v. } \\
\text { hinten) }\end{array}$ & & \\
\hline 17 & $\begin{array}{l}23.47- \\
24.31\end{array}$ & $\begin{array}{l}\text { Bade- } \\
\text { zimmer im } \\
\text { Herrenhaus }\end{array}$ & F, Haushälterin & $\begin{array}{l}\text { Haushälterin } \\
\text { macht einen } \\
\text { Hygiene-Check bei } \\
\text { F }\end{array}$ & & $\begin{array}{l}\text { Vogelge- } \\
\text { zwitscher aus } \\
\text { dem Garten }\end{array}$ & $\begin{array}{l}\text { 1. close-up (F, } \\
\text { Hände d. Haush.), } \\
\text { normal camera } \\
\text { height, statische } \\
\text { Kamera }\end{array}$ & $\begin{array}{l}\text { beige/ Licht } \\
\text { von draußen } \\
\text { (Fenster) }\end{array}$ & $\begin{array}{l}\text { Badezimmer, } \\
\text { Fließen ander } \\
\text { Wand, keine } \\
\text { Möbel, Vorhang } \\
\text { im Hintergrund }\end{array}$ \\
\hline 18 & $\begin{array}{l}24.32- \\
24.55\end{array}$ & D. & F, Haushälterin & $\begin{array}{l}\text { Haushälterin zeigt } \\
\text { ihr das Zimmer, } \\
\text { ihre Uniform und } \\
\text { Hygieneartikel }\end{array}$ & & $\begin{array}{l}\text { Vogelge- } \\
\text { zwitscher aus } \\
\text { dem Garten }\end{array}$ & $\begin{array}{l}\text { 1. medium long } \\
\text { shot (Bett) } \\
\text { 2. mediumshot( } F \text {, } \\
\text { Haush.), normal } \\
\text { camera height }\end{array}$ & $\begin{array}{l}\text { beige, braun, } \\
\text { rosa/ } \\
\text { schummriges } \\
\text { Licht von } \\
\text { draußen (es } \\
\text { wird dunkel) }\end{array}$ & $\begin{array}{l}\text { einfaches Zimmer } \\
\text { in altmodischem } \\
\text { Einrichtungsstil: } \\
\text { schlichtes Bett, } \\
\text { dunkles Holz, } \\
\text { Vorhang (dadurch } \\
\text { schummriges } \\
\text { Licht) }\end{array}$ \\
\hline 19 & $\begin{array}{l}24.56- \\
26.08\end{array}$ & E. & $\begin{array}{l}\text { F } \\
\text { Uniform: grüne } \\
\text { Strickjacke, } \\
\text { hellgrün/ beiger } \\
\text { Rock, darunter } \\
\text { trägt F immer } \\
\text { noch ihre Hose } \\
\text { mit } \\
\text { hochgewickelten } \\
\text { Hosenbeinen, } \\
\text { Haare im Zopf }\end{array}$ & $\begin{array}{l}\text { F wartet in steifer, } \\
\text { angespannter } \\
\text { Haltung in der } \\
\text { Küche bis die } \\
\text { Glocke läutet, } \\
\text { steht ruckartig auf, } \\
\text { balltdieHände zu } \\
\text { Fäusten }\end{array}$ & & $\begin{array}{l}\text { Klaviermusik } \\
\text { aus Klavier- } \\
\text { zimmer, Eule, } \\
\text { Grillen, Glocke }\end{array}$ & $\begin{array}{l}\text { 1. medium long } \\
\text { shot }(F) \text {, low shot, } \\
\text { statische Kamera }\end{array}$ & $\begin{array}{l}\text { orange, braun, } \\
\text { grün/ warmes } \\
\text { Licht von } \\
\text { Lampe } \\
\text { (draußen } \\
\text { dunkel) }\end{array}$ & $\begin{array}{l}\text { Küche: } \\
\text { Einrichtung aus } \\
\text { vergangenen } \\
\text { Zeiten, dunkles } \\
\text { Holz, dunkle } \\
\text { Bodenfließen in } \\
\text { Zickzackmuster, } \\
\text { gelbe } \\
\text { Wandkacheln, } \\
\text { alter Fernseher }\end{array}$ \\
\hline 20 & $\begin{array}{l}26.09- \\
27.34\end{array}$ & $\begin{array}{l}\text { Weg durch } \\
\text { das } \\
\text { Herrenhaus }\end{array}$ & $\mathrm{F}$ & $\begin{array}{l}\text { F sucht den Weg } \\
\text { durch das } \\
\text { Herrenhaus zu } \\
\text { Aída's } \\
\text { Schlafzimmer, } \\
\text { diese ruft Fimmer } \\
\text { beim falschen } \\
\text { Namen }\end{array}$ & Labyrinth & $\begin{array}{l}\text { Knirschen der } \\
\text { Schwingtür und } \\
\text { des Holzbodens }\end{array}$ & $\begin{array}{l}\text { 1. medium shot, } \\
\text { normal camera } \\
\text { height } \\
\text { 2. medium long } \\
\text { shot ( } v \text {. mehreren } \\
\text { Räumen im Haus), } \\
\text { dabei befindet sich } \\
\text { die Kamera immer }\end{array}$ & $\begin{array}{l}\text { grün, beige, } \\
\text { braun/ } \\
\text { schattig, } \\
\text { halbdunkel, } \\
\text { Licht von } \\
\text { Lampen } \\
\text { (stellenweise) }\end{array}$ & $\begin{array}{l}\text { Teure, wertvolle, } \\
\text { antike Möbel, } \\
\text { (Kolonialstil), } \\
\text { Teppiche, } \\
\text { Gemälde, große, } \\
\text { bunte Fenster, } \\
\text { Stehlampen, im } \\
\text { Flur einGemälde }\end{array}$ \\
\hline
\end{tabular}




\begin{tabular}{|c|c|c|c|c|c|c|c|c|c|}
\hline & & & & & & & $\begin{array}{l}\text { schon im Raum und } \\
\text { F tritt in denjenigen } \\
\text { Raum ein } \\
\text { 3. close-up (F), } \\
\text { Hinterherfahrt }\end{array}$ & & $\begin{array}{l}\text { von Maria und } \\
\text { Jesus, dunkle } \\
\text { Flure ohne } \\
\text { Ausgang: } \\
\text { Labyrinth }\end{array}$ \\
\hline 21 & $\begin{array}{l}27.35- \\
28.39\end{array}$ & $\mathrm{~F}$. & $\begin{array}{l}\text { F, Aída } \\
\text { Aída: Lederstiefel, } \\
\text { lockere } \\
\text { Buntfaltenhose } \\
\text { bis zum Knie, } \\
\text { braunes Oberteil, } \\
\text { lange Perlenkette }\end{array}$ & $\begin{array}{l}\text { Aída bohrt ein } \\
\text { Loch in die Wand, } \\
\text { F ist ihr behilflich, } \\
\text { sie sieht ihr } \\
\text { Spiegelbild mit } \\
\text { Bohrmaschine im } \\
\text { Bild eines Offiziers } \\
\text { und bekommt } \\
\text { Nasenbluten (sieht } \\
\text { aus als trüge sie } \\
\text { eine Waffe), sie } \\
\text { rennt in die Küche }\end{array}$ & & $\begin{array}{l}\text { Bohrmaschine, } \\
\text { Türklopfen, } \\
\text { Klopfen, } \\
\text { schnelle Schritte } \\
\text { auf Holzboden }\end{array}$ & $\begin{array}{l}\text { 1. close-up (F), } \\
\text { normal camera } \\
\text { height } \\
\text { 2. medium long } \\
\text { shot (Aída) } \\
\text { 3. extreme close-up } \\
\text { (Bild) mit F's } \\
\text { Spiegelbild darin } \\
\text { (close shot) } \\
\text { 4. close shot (Aída), } \\
\text { normal camera } \\
\text { height } \\
\text { 5. medium long } \\
\text { shot, Kamera } \\
\text { befindet sich im } \\
\text { Raum, F tritt ein }\end{array}$ & $\begin{array}{l}\text { braun, } \\
\text { khakifarben } \\
\text { (Militär)/ } \\
\text { orangefarbene } \\
\text { Lampe, sonst } \\
\text { dunkel }\end{array}$ & $\begin{array}{l}\text { großes, elegantes } \\
\text { Doppelbett aus } \\
\text { dunklem Holz, } \\
\text { darüber ist die } \\
\text { Wand vollerBilder } \\
\text { in teuer } \\
\text { aussehenden } \\
\text { Bilderrahmen }\end{array}$ \\
\hline 22 & $\begin{array}{l}28.40- \\
29.20\end{array}$ & E. & F, Aída & $\begin{array}{l}\text { F putzt sich die } \\
\text { Nase und beruhigt } \\
\text { sich mit Gesang, } \\
\text { Aída beobachtet } \\
\text { sie }\end{array}$ & & $\begin{array}{l}\text { Gesang } F_{\text {, }} \\
\text { Wasser }\end{array}$ & $\begin{array}{l}\text { 1. close shot(F), } \\
\text { high shot } \\
\text { 2. medium long } \\
\text { shot (Aída), low } \\
\text { shot }\end{array}$ & $\begin{array}{l}\text { braun, orange/ } \\
\text { schummriges } \\
\text { Licht, Aída im } \\
\text { Schatten }\end{array}$ & $\begin{array}{l}\text { Fsteht gebeugt an } \\
\text { der Spüle, Aída } \\
\text { beobachtetsie } \\
\text { von der } \\
\text { Schwingtür aus }\end{array}$ \\
\hline 23 & $\begin{array}{l}29.21- \\
30.08\end{array}$ & D. & $F$ & $\begin{array}{l}\text { F schneidet die } \\
\text { Wurzeln von der } \\
\text { Kartoffel mit einer } \\
\text { Nagelschere, F } \\
\text { zittert und ist } \\
\text { verkrampft dabei, }\end{array}$ & Kartoffel & $\begin{array}{l}\text { Gesang F (zur } \\
\text { Beruhigung), } \\
\text { Grillen aus dem } \\
\text { Garten, } \\
\text { Abschneiden } \\
\text { der Wurzel }\end{array}$ & $\begin{array}{l}\text { 1. close shot (F), } \\
\text { normal camera } \\
\text { height } \\
\text { 2. close shot } \\
\text { (Nagelschere, F } \\
\text { Hand) } \\
\text { 3. extreme close-up } \\
\text { (Füße, Wurzel), } \\
\text { extreme high shot }\end{array}$ & $\begin{array}{l}\text { türkis/ } \\
\text { Tageslicht vom } \\
\text { Fenster }\end{array}$ & $\begin{array}{l}\text { F sitzt mit dem } \\
\text { Gesicht zum } \\
\text { Fenster, nebenihr } \\
\text { liegen die } \\
\text { Hygieneartikel } \\
\text { geordnet auf dem } \\
\text { Bett, einfache, } \\
\text { kahle Einrichtung } \\
\text { im Vergleich zum } \\
\text { Rest des Hauses }\end{array}$ \\
\hline
\end{tabular}




\begin{tabular}{|c|c|c|c|c|c|c|c|c|}
\hline \multicolumn{9}{|c|}{3 Sekunden Schwarzblende } \\
\hline 24 & $\begin{array}{l}30.12- \\
30.20\end{array}$ & D. & $\mathrm{F}$ & $\begin{array}{l}\text { Fwartet bis es am } \\
\text { Eingangstor } \\
\text { klingelt }\end{array}$ & $\begin{array}{l}\text { Vogelge- } \\
\text { zwitscher, } \\
\text { Klingel }\end{array}$ & $\begin{array}{l}\text { 1. mediumshot, } \\
\text { normal camera } \\
\text { height, statische } \\
\text { Kamera }\end{array}$ & $\begin{array}{l}\text { türkis/ } \\
\text { Tageslicht vom } \\
\text { Fenster }\end{array}$ & $\begin{array}{l}\text { Fsitztmit Rücken } \\
\text { zum Fenster mit } \\
\text { Fäusten im } \\
\text { Schoss, } \\
\text { angespannte } \\
\text { Körperhaltung }\end{array}$ \\
\hline 25 & $\begin{array}{l}30.21- \\
31.31\end{array}$ & C. & $\begin{array}{l}\text { F, Gärtner Noé } \\
\text { Noé: } \\
\text { Alltagskleider, } \\
\text { Fahrrad }\end{array}$ & $\begin{array}{l}\text { Flässt den Gärtner } \\
\text { eintreten, sie läuft } \\
\text { schnell zurück ins } \\
\text { Haus ohne ihn } \\
\text { anzuschauen } \\
\text { (geballte Fäuste) }\end{array}$ & $\begin{array}{l}\text { Vogelge- } \\
\text { zwitscher, } \\
\text { Stimmen- } \\
\text { gewirr vom } \\
\text { Markt }\end{array}$ & $\begin{array}{l}\text { 1. medium long } \\
\text { shot, Kamera auf } \\
\text { Position, F tritt ins } \\
\text { Bild } \\
\text { 2. close-up (F's } \\
\text { halbes Gesicht im } \\
\text { Fenster) / subj. } \\
\text { Kamera (Persp. } \\
\text { Noé) } \\
\text { 3. closeshot(Noé)/ } \\
\text { subj. Kamera } \\
\text { (Persp. F) } \\
\rightarrow \text { Wdh. } 2+3 \\
\text { 4. medium shot (F } \\
\text { tritt ins Bild), } \\
\text { statische Kamera, F } \\
\text { geht, Tor öffnet, } \\
\text { full shot (Noé) } \\
\text { 5. medium long } \\
\text { shot (F entfernt } \\
\text { sich) }\end{array}$ & $\begin{array}{l}\text { braun, } \\
\text { steingrau, } \\
\text { grün/ } \\
\text { Tageslicht }\end{array}$ & $\begin{array}{l}\text { Steinmauer und } \\
\text { halbes } \\
\text { Eingangstor, F } \\
\text { schaut durch } \\
\text { kleineKlappezum } \\
\text { Markt hinaus, } \\
\text { passtsich farblich } \\
\text { perfekt an ihre } \\
\text { Umgebung an }\end{array}$ \\
\hline 26 & $\begin{array}{l}31.32- \\
31.46\end{array}$ & $\begin{array}{l}\text { Lange Treppe } \\
\text { zum } \\
\text { Armenviertel } \\
\text { Manchay }\end{array}$ & $\begin{array}{l}\text { F, Máxima } \\
\text { Máxima: enge } \\
\text { Kleidung, } \\
\text { schlenkert mit } \\
\text { ihrer Handtasche } \\
\text { F: lockere } \\
\text { Kleidung, }\end{array}$ & $\begin{array}{l}\text { F und Máxima } \\
\text { laufen die lange } \\
\text { Treppe hoch }\end{array}$ & $\begin{array}{l}\text { Straßen- } \\
\text { geräusche, } \\
\text { Wind }\end{array}$ & $\begin{array}{l}\text { 1. long shot, low } \\
\text { shot } \\
\text { 2. extreme long } \\
\text { shot, low shot }\end{array}$ & $\begin{array}{l}\text { sandfarben, } \\
\text { himmelblau/ } \\
\text { Tageslicht } \\
\text { (sonnig) }\end{array}$ & $\begin{array}{l}\text { nicht endende } \\
\text { Treppein Bergen, } \\
\text { unten Straße, } \\
\text { oben Statue }\end{array}$ \\
\hline
\end{tabular}




\begin{tabular}{|c|c|c|c|c|c|c|c|c|c|}
\hline 27 & $\begin{array}{l}31.47- \\
34.29\end{array}$ & Manchay & $\begin{array}{l}\text { Brautpaar, Gäste, } \\
\text { Tío, Tante, F } \\
\text { etwas maskulin } \\
\text { wirkende Braut } \\
\text { (kräftig, groß, mit } \\
\text { markanten } \\
\text { Gesichtszügen } \\
\text { und Tattoo am } \\
\text { Arm: üppiges } \\
\text { Kleid, eher } \\
\text { schmächtiger, } \\
\text { kleiner Bräutigam } \\
\text { (grinsend) } \\
\text { F und Familie in } \\
\text { Uniform } \\
\text { (schwarze Hose, } \\
\text { gelbes Hemd, } \\
\text { Weste undFliege } \\
\rightarrow \text { maskulin) } \\
\text { Band: pink und } \\
\text { silber } \rightarrow \text { auffällig }\end{array}$ & Hochzeitsfeier & Tauben & $\begin{array}{l}\text { fröhliche Musik } \\
\text { (Liveband), } \\
\text { Applaus, } \\
\text { Glückwünsche }\end{array}$ & $\begin{array}{l}\text { 1. medium full shot } \\
\text { (bis Knie), } \\
\text { Vorwegfahrt, close- } \\
\text { up, Vorwegfahrt, } \\
\text { Kamera kreist um } \\
\text { das Brautpaar } \\
\text { 2. medium full shot } \\
\text { (alle) } \\
\text { 3. Kamera } \\
\text { schwenkt über } \\
\text { Essen, close-up } \\
\text { (essende Frauen) } \\
\text { 4. medium shot } \\
\text { (Brautpaar, } \\
\text { Tauben) } \\
\text { 5. close shot } \\
\text { (Gäste) } \\
\text { 6. medium long } \\
\text { shot (Tanzfläche) }\end{array}$ & $\begin{array}{l}\text { rosa, weiß, lila, } \\
\text { sandfarben/ } \\
\text { Tageslicht }\end{array}$ & $\begin{array}{l}\text { kitschige } \\
\text { Hochzeitsdeko, } \\
\text { Brautpaar kommt } \\
\text { von Treppe } \\
\text { herunter, karge } \\
\text { Landschaft } \\
\text { außenherum }\end{array}$ \\
\hline 28 & $\begin{array}{l}34.30- \\
35.36\end{array}$ & $\begin{array}{l}\text { Manchay } \\
\text { (Abseits der } \\
\text { Feier) }\end{array}$ & F, Tío & $\begin{array}{l}\text { TíosetztFein } \\
\text { Ultimatum }\end{array}$ & & $\begin{array}{l}\text { fröhliche Musik } \\
\text { im Hintergrund, } \\
\text { Wind }\end{array}$ & $\begin{array}{l}\text { 1. full shot }(F, \text { tío }) v \text {. } \\
\text { Seite } \\
\text { 2. full shot }(F, \text { tío }) v \text {. } \\
\text { vorn }\end{array}$ & $\begin{array}{l}\text { türkis, rosa, } \\
\text { lila, schwarz/ } \\
\text { Tageslicht }\end{array}$ & $\begin{array}{l}\text { Tío und F werden } \\
\text { optisch von der } \\
\text { Hochzeitsdeko } \\
\text { getrennt } \\
\text { (Stoffbahnen) }\end{array}$ \\
\hline 29 & $\begin{array}{l}35.37- \\
36.05\end{array}$ & $\begin{array}{l}\text { "Busbahnhof" } \\
\text { in Manchay }\end{array}$ & $\begin{array}{l}\text { F, Severina, } \\
\text { Ticketverkäufer } \\
\text { einige Frauen in } \\
\text { traditionellen } \\
\text { Quechua- } \\
\text { Trachten }\end{array}$ & $\begin{array}{l}\text { F fragt nach } \\
\text { Transportkosten, } \\
\text { sie wird } \\
\text { unfreundlich } \\
\text { abgewiesen }\end{array}$ & & $\begin{array}{l}\text { Stimmen- } \\
\text { gewirr }\end{array}$ & $\begin{array}{l}\text { 1. mediumlong } \\
\text { shot } \\
\text { 2. close shot } \\
\text { (Verk.), Schuss } \\
\text { 3. close shot (F, } \\
\text { Sev.), Gegenschuss } \\
\rightarrow \text { mehrmals Wdh. } \\
2+3\end{array}$ & $\begin{array}{l}\text { sandfarben/ } \\
\text { Tageslicht }\end{array}$ & $\begin{array}{l}\text { BergeundStraße, } \\
\text { am linken Rand } \\
\text { kleines Häuschen } \\
\text { (Ticketstand) }\end{array}$ \\
\hline
\end{tabular}




\begin{tabular}{|c|c|c|c|c|c|c|c|c|}
\hline 30 & $\begin{array}{l}36.06- \\
36.50\end{array}$ & $\begin{array}{l}\text { Lange Treppe } \\
\text { (von oben) }\end{array}$ & $\begin{array}{l}\text { F, Severina, } \\
\text { Bewohner }\end{array}$ & $\begin{array}{l}\mathrm{F} \text { ist böse auf } \\
\text { Severina (Sarg), } \\
\text { läuft Richtung } \\
\text { Treppe, bekommt } \\
\text { Angst alleine die } \\
\text { Treppe } \\
\text { hinunterzugehen, } \\
\text { weil ein Mann ihr } \\
\text { entgegenkommt, } \\
\text { fordert Severina } \\
\text { ohne Worte auf, } \\
\text { zu ihr zu kommen } \\
\text { (wirkt sehr } \\
\text { egoistisch, vor } \\
\text { allem, weil } \\
\text { Severina } \\
\text { hochschwanger } \\
\text { ist) }\end{array}$ & \begin{tabular}{l|} 
Straßen- \\
geräusche, \\
hallende \\
Kinderstimmen, \\
Schritte auf der \\
Treppe
\end{tabular} & $\begin{array}{l}\text { 1. medium long } \\
\text { shot, low shot, } F+ \\
\text { Sev. laufen auf } \\
\text { Kamera zu } \\
\text { 2. medium long } \\
\text { shot ( } F, \text { Mann) / } \\
\text { subj. Kamera } \\
\text { (Persp. Sev.), high } \\
\text { shot } \\
\text { 3. medium full shot } \\
\text { (Sev.), low shot } \\
\text { 4. medium long } \\
\text { shot ( } F \text {, Mann) / } \\
\text { subj. Kamera } \\
\text { (Persp. Sev.), high } \\
\text { shot } \\
\text { 5. medium shot } \\
\text { (Sev.), low shot } \\
\text { 6. medium long } \\
\text { shot, high shot ( } F \text {, } \\
\text { Sev. entfernen sich, } \\
\text { Mann nähertsich) }\end{array}$ & $\begin{array}{l}\text { sandfarben, } \\
\text { beige/ } \\
\text { Tageslicht }\end{array}$ & $\begin{array}{l}\text { lange, steile } \\
\text { Treppe von oben, } \\
\text { unten } \\
\text { Armenviertel und } \\
\text { Berge im } \\
\text { Hintergrund }\end{array}$ \\
\hline 31 & $\begin{array}{l}36.51- \\
37.22\end{array}$ & C. & $F$ & $\begin{array}{l}\text { Fschaut sich die } \\
\text { Scherben umdas } \\
\text { kaputte Klavieran } \\
\text { und hältsich eine } \\
\text { Scherbe an die } \\
\text { Nase, soalswürde } \\
\text { sie daran riechen }\end{array}$ & $\begin{array}{l}\text { meditativer } \\
\text { Klangton, } \\
\text { Vogelge- } \\
\text { zwitscher }\end{array}$ & $\begin{array}{l}\text { 1. medium long } \\
\text { shot (Klavier), } \\
\text { Kamera auf } \\
\text { Position, F tritt ins } \\
\text { Bild } \\
\text { 2. full shot, high } \\
\text { shot (Klavier, F, } \\
\text { Splitter) }\end{array}$ & \begin{tabular}{|l|} 
sandfarben, \\
grün, bunt \\
(Glassplitter) / \\
Tageslicht
\end{tabular} & $\begin{array}{l}\text { Garten: auf dem } \\
\text { Kiesweg liegt das } \\
\text { kaputte Klavier, } \\
\text { drum herum } \\
\text { bunte Glassplitter }\end{array}$ \\
\hline 32 & $\begin{array}{l}37.23- \\
38.00\end{array}$ & $\mathrm{~F}$. & Aída, Sohn, F & $\begin{array}{l}\text { Aída und Sohn } \\
\text { unterhalten sich } \\
\text { ohne Blickkontakt, } \\
\text { F bringt Tee }\end{array}$ & & $\begin{array}{l}\text { 1. medium long } \\
\text { shot (Aída, Sohn), } \\
\text { normal camera } \\
\text { height } \\
\text { 2. full shot (F } \\
\text { bewegt sich auf }\end{array}$ & $\begin{array}{l}\text { braun, beige/ } \\
\text { oranges Licht } \\
\text { von Lampe }\end{array}$ & $\begin{array}{l}\text { riesiges, } \\
\text { pompöses Bett in } \\
\text { der Mitte }\end{array}$ \\
\hline
\end{tabular}




\begin{tabular}{|c|c|c|c|c|c|c|c|c|}
\hline & & & & & & $\begin{array}{l}\text { Kamera zu), normal } \\
\text { camera height } \\
\text { 3. medium long } \\
\text { shot (Aída), normal } \\
\text { camera height } \\
\text { 4. medium full shot } \\
\text { (F nähert sich } \\
\text { weiter) }\end{array}$ & & \\
\hline 33 & $\begin{array}{l}38.01- \\
39.26\end{array}$ & E. & $\begin{array}{l}\text { F, Aída } \\
\text { Aída: Schmuck, } \\
\text { Perlenkette, } \\
\text { elegante Bluse }\end{array}$ & $\begin{array}{l}\text { F schaut lächelnd } \\
\text { TV, Aída fordert } \\
\text { sie zum Singen } \\
\text { auf, F kann nicht }\end{array}$ & $\begin{array}{l}\text { Fernseher, } \\
\text { Knarren derTür, } \\
\text { Pfeifen des } \\
\text { Wasserkessels }\end{array}$ & $\begin{array}{l}\text { 1. close shot (F), } \\
\text { Aída setzt sich zu } \\
\text { ihr } \\
\text { 2. extreme close-up } \\
\text { (TV) } \\
\text { 3. close shot ( } F \text {, } \\
\text { Aída) } \\
\text { 4. close-up } \\
\text { (Gesichterv. Seite), } \\
\text { Fokus Aída, dann } \\
\text { Fokus } F \\
\text { 5. close shot (F), } \\
\text { Aída geht } \\
\text { 6. close-up } \\
\text { (Teekessel) } \\
\text { normal camera } \\
\text { height }\end{array}$ & $\begin{array}{l}\text { türkis, } \\
\text { rotbraun, gelb/ } \\
\text { warmes Licht } \\
\text { von Lampe }\end{array}$ & $\begin{array}{l}\text { F sitzt vorm TV, } \\
\text { Küche im } \\
\text { Hintergrund, Aída } \\
\text { setztsich aufden } \\
\text { Stuhl nebenF }\end{array}$ \\
\hline 34 & $\begin{array}{l}39.27- \\
40.47\end{array}$ & $\mathrm{~F}$. & F, Aída & $\begin{array}{l}\text { Fbringt Tee, Aída } \\
\text { und F sammeln } \\
\text { Perlen vom Boden } \\
\text { auf, Aída schlägt } \\
\text { ihreinen Paktvor } \\
\text { (eine Perle für } \\
\text { jedes Lied) }\end{array}$ & $\begin{array}{l}\text { Wasserhahn, } \\
\text { auf Fliesen } \\
\text { fallendePerlen }\end{array}$ & $\begin{array}{l}\text { 1. medium long } \\
\text { shot ( } F \text { nähert sich), } \\
\text { normal camera } \\
\text { height } \\
\text { 2. medium shot } \\
\text { (Aída), Schuss } \\
\text { 3. close shot (F), } \\
\text { Gegenschuss } \\
\rightarrow \text { Wdh. } 2+3 \\
\text { 4. Kamera auf } \\
\text { Position, Frauen }\end{array}$ & $\begin{array}{l}\text { türkis/ } \\
\text { schummriges } \\
\text { Licht, im Bad } \\
\text { grelles } \\
\text { Neonlicht }\end{array}$ & $\begin{array}{l}\text { helles Bad mit } \\
\text { großem Spiegel, } \\
\text { beide Frauen } \\
\text { nähern sich } \\
\text { langsam von } \\
\text { rechts und links, } \\
\text { beide auf allen } \\
\text { Vieren, F bewegt } \\
\text { sich sehr langsam, } \\
\text { wie ein scheues } \\
\text { Raubtier }\end{array}$ \\
\hline
\end{tabular}




\begin{tabular}{|c|c|c|c|c|c|c|c|c|c|}
\hline & & & & & & & $\begin{array}{l}\text { bewegen sich } \\
\text { kriechend von } \\
\text { beiden Seiten ins } \\
\text { Bild, close shot }\end{array}$ & & \\
\hline 35 & $\begin{array}{l}40.48- \\
41.35\end{array}$ & C. & $\begin{array}{l}\text { F, Noé } \\
\text { F: Uniform, Haare } \\
\text { im Zopf }\end{array}$ & $\begin{array}{l}\text { F lässt Noé } \\
\text { eintreten, sie } \\
\text { sprechen auf } \\
\text { Quechua (erster } \\
\text { Kontakt) }\end{array}$ & & $\begin{array}{l}\text { meditativer } \\
\text { Klangton, } \\
\text { Vogelge- } \\
\text { zwitscher, } \\
\text { Knirschen der } \\
\text { Glasscherben }\end{array}$ & $\begin{array}{l}\text { 1. close shot }(\mathrm{F}), \\
\text { normal camera } \\
\text { height } \\
\text { 2. extreme close-up } \\
\text { (F's Augen/ Nase } \\
\text { im Fenster)/ subj. } \\
\text { Kamera (Persp. } \\
\text { Noé) } \\
\text { 3. extreme close-up } \\
\text { (Hand Noé) / subj. } \\
\text { Kamera (Persp. F) } \\
\text { 4. full shot, high } \\
\text { shot (F, Noé, } \\
\text { Klavier) }\end{array}$ & $\begin{array}{l}\text { türkis, } \\
\text { steingrau, } \\
\text { grün, bunt } \\
\text { (Glassplitter)/ } \\
\text { Tageslicht }\end{array}$ & $\begin{array}{l}\text { Fdrückt sich an } \\
\text { die Steinmauer } \\
\text { (wie Chamäleon), } \\
\text { beide umrunden } \\
\text { das kaputte } \\
\text { Klavier und } \\
\text { sammeln ein paar } \\
\text { Glasscherben auf }\end{array}$ \\
\hline 36 & $\begin{array}{l}41.36- \\
45.11\end{array}$ & B. & $\begin{array}{l}\text { Verlobte, Gäste, F, } \\
\text { Primo von Marcos } \\
\text { alle sind schick } \\
\text { angezogen, } \\
\text { Máxima sehr } \\
\text { weiblich, } \\
\text { herausgeputzt, F } \\
\text { hellblauer } \\
\text { Hosenanzug, } \\
\text { offene Haare }\end{array}$ & $\begin{array}{l}\text { Verlobungsfeier, } \\
\text { Máxima schält } \\
\text { Kartoffel, sie } \\
\text { bekommt den } \\
\text { Ring, Amadeo, ein } \\
\text { Bekannter, setzt } \\
\text { sich zu F und } \\
\text { Jonathan, } \\
\text { Jonathan holt } \\
\text { Milch für das Baby } \\
\text { und F wirft ihm } \\
\text { einenbösen Blick } \\
\text { zu, weil er siemit } \\
\text { Amadeo allein } \\
\text { lässt, F bekommt } \\
\text { seltsamen } \\
\text { Anmachspruch, sie }\end{array}$ & Kartoffel & $\begin{array}{l}\text { fröhliche Musik, } \\
\text { Schälen der } \\
\text { Kartoffel, } \\
\text { gackernde } \\
\text { Hühner, } \\
\text { Kindergeschrei }\end{array}$ & $\begin{array}{l}\text { 1. close-up } \\
\text { (Kartoffel in } \\
\text { Máxima's Händen) } \\
\text { 2. extreme close-up } \\
\text { (Schale zw. ihren } \\
\text { Füßen), high shot } \\
\text { 3. close shot } \\
\text { (Max.), normal } \\
\text { camera height } \\
\text { 4. close-up (Schale } \\
\text { zw. ihren Füßen), } \\
\text { high shot } \\
\text { 5. close-up (tío mit } \\
\text { Schale in der } \\
\text { Hand), normal } \\
\text { camera height }\end{array}$ & $\begin{array}{l}\text { rosa, braun, } \\
\text { sandfarben, } \\
\text { steingrau/ } \\
\text { Tageslicht }\end{array}$ & $\begin{array}{l}\text { Stuhlkreis im Hof } \\
\text { vor dem Haus des } \\
\text { Onkels, kitschige } \\
\text { Dekoration }\end{array}$ \\
\hline
\end{tabular}




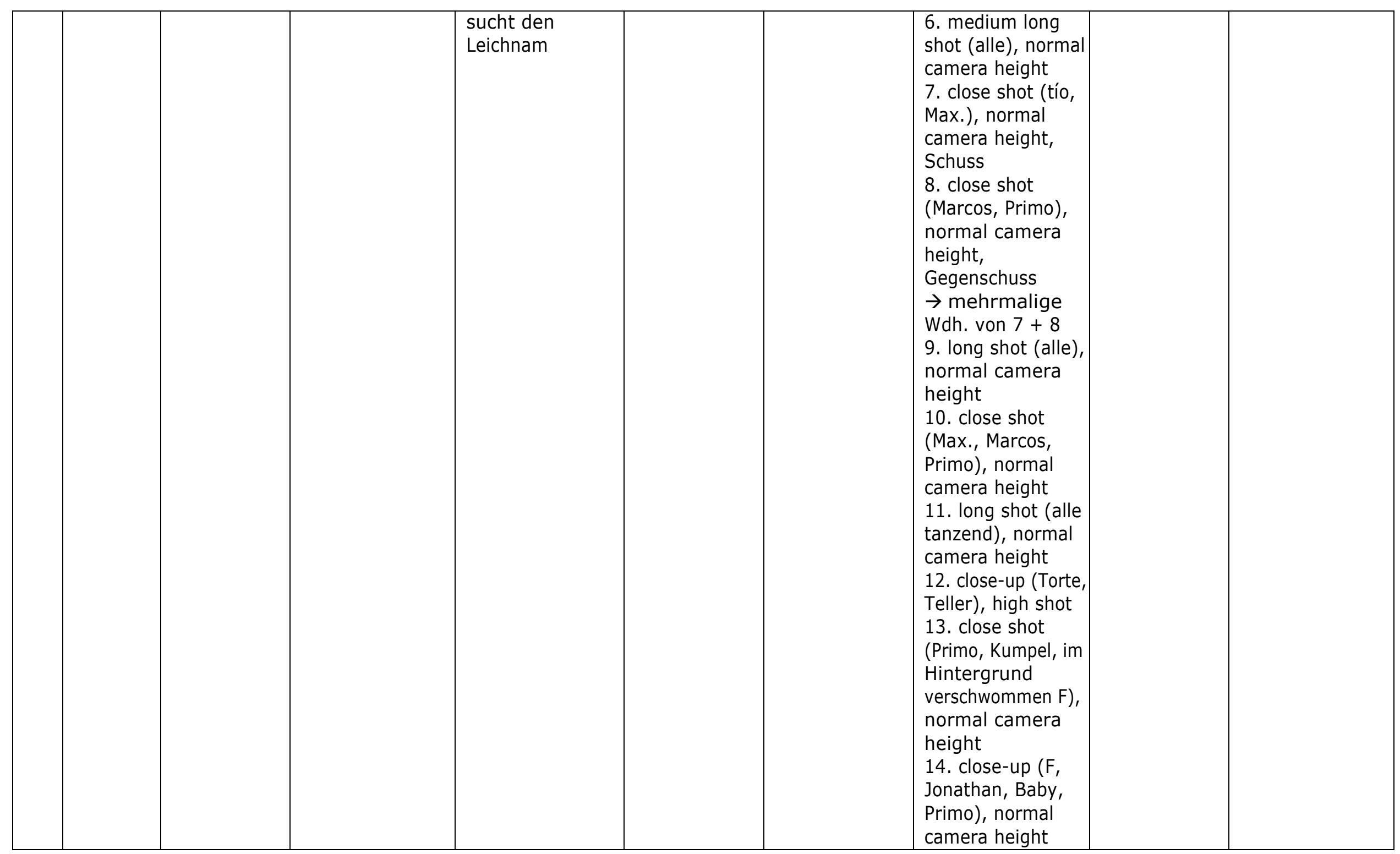




\begin{tabular}{|c|c|c|c|c|c|c|c|c|c|}
\hline & & & & & & & $\begin{array}{l}\text { 15. close-up (F), } \\
\text { bewegt sich auf } \\
\text { Kamera zu } \\
\text { 16. full shot } \\
\text { (Brautkleid auf } \\
\text { Perpetua's Bett)/ } \\
\text { subj. Kamera } \\
\text { (Persp. F) } \\
\text { 17. close-up (F), } \\
\text { entfernt sich von } \\
\text { Kamera, Schwenk } \\
\text { auf Tanzfläche, } \\
\text { mediumlong shot, } \\
\text { high shot }\end{array}$ & & \\
\hline 37 & $\begin{array}{l}45.12- \\
45.38\end{array}$ & A. & F, Leichnam & $\begin{array}{l}\text { F verschiebt das } \\
\text { Bett, setztsich } \\
\text { neben den } \\
\text { Leichnam }\end{array}$ & Mumie & $\begin{array}{l}\text { Schleifen des } \\
\text { Bettes über } \\
\text { Betonboden }\end{array}$ & $\begin{array}{l}\text { 1. close shot } \\
\text { (Brautkleid, } \\
\text { Leichnam), extreme } \\
\text { high shot } \\
\text { 2. medium long } \\
\text { shot (Leichnam, F } \\
\text { aufBoden), normal } \\
\text { camera height }\end{array}$ & $\begin{array}{l}\text { weiß, blau/ } \\
\text { Tageslicht von } \\
\text { draußen }\end{array}$ & $\begin{array}{l}\text { im Zimmer: für } \\
\text { einen kurzen } \\
\text { Moment sieht es } \\
\text { so aus, als träge } \\
\text { die tote Mutter } \\
\text { das Brautkleid }\end{array}$ \\
\hline 38 & $\begin{array}{l}45.39- \\
45.55\end{array}$ & $E$. & $\begin{array}{l}\text { Aída } \\
\text { (passive Präsenz } \\
\text { von F) }\end{array}$ & $\begin{array}{l}\text { Aída führt } \\
\text { Telefonat über } \\
\text { Konzert (läuft } \\
\text { etwas nervös hin } \\
\text { und her) }\end{array}$ & & $\begin{array}{l}\text { Klappern der } \\
\text { Werkzeuge aus } \\
\text { Wohn- } \\
\text { zimmer }\end{array}$ & $\begin{array}{l}\text { 1. full shot (Aída, } \\
\text { Blick durch Tür), } \\
\text { normal camera } \\
\text { height (eventuell } \\
\text { subj. Kamera/ } \\
\text { Persp. F) }\end{array}$ & $\begin{array}{l}\text { braun gelb/ } \\
\text { Tageslicht von } \\
\text { draußen }\end{array}$ & \\
\hline 39 & $\begin{array}{l}45.56- \\
47.01\end{array}$ & $\begin{array}{l}\text { Wohnraum } \\
\text { im } \\
\text { Herrenhaus }\end{array}$ & $\begin{array}{l}2 \text { Bauarbeiter, F, } \\
\text { Aída }\end{array}$ & \begin{tabular}{l|} 
Bauarbeiter \\
messen das \\
Fenster aus, Aída \\
verhandelt den \\
Preis der \\
Reparatur, F wird \\
von Lehrling \\
angesprochen, F
\end{tabular} & & $\begin{array}{l}\text { Werkzeuge, } \\
\text { Eule, Vogelge- } \\
\text { zwitscher }\end{array}$ & $\begin{array}{l}\text { 1. close shot } \\
\text { (Bauarbeiter), } \\
\text { normal camera } \\
\text { height } \\
\text { 2. medium full shot } \\
\text { (Fbis Knie), normal } \\
\text { camera height }\end{array}$ & $\begin{array}{l}\text { grün, türkis, } \\
\text { braun/ } \\
\text { Tageslicht von } \\
\text { draußen }\end{array}$ & $\begin{array}{l}\text { Pflanzen ragen } \\
\text { durch das kaputte } \\
\text { Fenster in den } \\
\text { Wohnraum }\end{array}$ \\
\hline
\end{tabular}




\begin{tabular}{|c|c|c|c|c|c|c|c|c|}
\hline & & & & $\begin{array}{l}\text { meidet den Blick } \\
\text { und senkt den } \\
\text { Kopf }\end{array}$ & & $\begin{array}{l}\text { 3. medium long } \\
\text { shot (alle), normal } \\
\text { camera height } \\
\text { 4. close shot (Aída, } \\
\text { Bau.), normal } \\
\text { camera height } \\
\rightarrow \text { mehrmalige } \\
\text { Wdh. } 2+4 \text { (Aída } \\
\text { geht aus dem } \\
\text { Raum) }\end{array}$ & & \\
\hline 40 & $\begin{array}{l}47.02- \\
47.15\end{array}$ & D. & $F$ & $\begin{array}{l}\text { F sitzt auf dem } \\
\text { Bett, knöpft sich } \\
\text { die Jacke zu und } \\
\text { wartet bis es } \\
\text { klingelt }\end{array}$ & $\begin{array}{l}\text { Vogelge- } \\
\text { zwitscher, } \\
\text { Klingel }\end{array}$ & $\begin{array}{l}\text { 1. medium shot }(F) \text {, } \\
\text { normal camera } \\
\text { height }\end{array}$ & $\begin{array}{l}\text { beige, türkis/ } \\
\text { schummriges } \\
\text { Licht von } \\
\text { draußen }\end{array}$ & $\begin{array}{l}\text { F sitztmitdem } \\
\text { Rücken zum } \\
\text { Fenster auf dem } \\
\text { Bett }\end{array}$ \\
\hline 41 & $\begin{array}{l}47.16- \\
48.00\end{array}$ & C. & $\begin{array}{l}\text { F, Primo von } \\
\text { Marcos, Noé }\end{array}$ & $\begin{array}{l}\text { F öffnet das Tor } \\
\text { (erwartet Noé } \\
\text { erfreut), sie } \\
\text { erschrickt und } \\
\text { lehnt es ab, mit } \\
\text { dem Primo } \\
\text { heimzugehen, } \\
\text { lässt Noé } \\
\text { eintreten }\end{array}$ & $\begin{array}{l}\text { Öffnen des } \\
\text { Eingangstors, } \\
\text { Stimmen- } \\
\text { gewirr vom } \\
\text { Markt, Fahrrad }\end{array}$ & $\begin{array}{l}\text { 1. close shot (F), } \\
\text { normal camera } \\
\text { height, subj. } \\
\text { Kamera (Persp. } \\
\text { Primo) } \\
\text { 2. close shot } \\
\text { (Primo), normal } \\
\text { camera height, } \\
\text { subj. Kamera } \\
\text { (Persp. F) } \\
\rightarrow \text { mehrmalige } \\
\text { Wdh. } 1+2 \text { (Noé } \\
\text { geht durch beide } \\
\text { Bilder) }\end{array}$ & $\begin{array}{l}\text { grün, } \\
\text { steingrau, bunt } \\
\text { (Markt) / } \\
\text { Tageslicht }\end{array}$ & $\begin{array}{l}\text { Eingangstor als } \\
\text { Trennwand } \\
\text { zwischen Garten } \\
\text { und Markt }\end{array}$ \\
\hline 42 & $\begin{array}{l}48.01- \\
49.47\end{array}$ & E. & F, Noé & $\begin{array}{l}\text { F beobachtet Noé } \\
\text { durch das } \\
\text { Küchenfenster, F } \\
\text { gibt Noé ein Glas } \\
\text { Wasser, er bietet } \\
\text { ihr an sie nach } \\
\text { Hause zu begleiten }\end{array}$ & $\begin{array}{l}\text { Kirchen- } \\
\text { glocken, } \\
\text { Vogelge- } \\
\text { zwitscher }\end{array}$ & $\begin{array}{l}\text { 1. medium long } \\
\text { shot (Noé), high } \\
\text { shot, subj. Kamera } \\
\text { (Persp. F), Kamera } \\
\text { filmt durch Fenster }\end{array}$ & $\begin{array}{l}\text { grün, } \\
\text { steingrau, } \\
\text { braun/ } \\
\text { Tageslicht von } \\
\text { draußen }\end{array}$ & $\begin{array}{l}\text { aus der Küche } \\
\text { hinaus in den } \\
\text { Garten gefilmt }\end{array}$ \\
\hline
\end{tabular}




\begin{tabular}{|c|c|c|c|c|c|c|c|c|}
\hline & & & & $\begin{array}{l}\text { (Quechua), F lehnt } \\
\text { erst ab, dann } \\
\text { ändert sie ihre } \\
\text { Meinung(gehtzu } \\
\text { ihm in den Garten) }\end{array}$ & & $\begin{array}{l}\text { 2. close-up (F im } \\
\text { Profil), normal } \\
\text { camera height } \\
\rightarrow \text { Wdh. } 1+2 \\
\text { 3. medium shot (F } \\
\text { v. hinten), normal } \\
\text { camera height } \\
\text { 4. close shot(Noé), } \\
\text { normal camera } \\
\text { height } \\
\rightarrow \text { Wdh. } 3 \\
\text { 5. medium full shot } \\
\text { (beide, ohne Füße), } \\
\text { normal camera } \\
\text { height } \\
\text { 6. Kamera filmt } \\
\text { weiter von der } \\
\text { KücheausaberFist } \\
\text { nun hinter der } \\
\text { Glasscheibe, } \\
\text { mediumlong shot } \\
\text { (Noé, F nähert } \\
\text { sich), high shot }\end{array}$ & & \\
\hline 43 & $\begin{array}{l}49.48- \\
50.03\end{array}$ & $\begin{array}{l}\text { Lange Treppe } \\
\text { zum } \\
\text { Armenviertel } \\
\text { Manchay }\end{array}$ & $\begin{array}{l}\text { F, Noé } \\
\text { F: offenes Haar }\end{array}$ & $\begin{array}{l}\text { Noé und F steigen } \\
\text { die Treppe hinauf, } \\
\text { F läuft vor Noé } \\
\text { (=Vertrauen?) }\end{array}$ & $\begin{array}{l}\text { Straßen- } \\
\text { geräusche }\end{array}$ & $\begin{array}{l}\text { 1. long shot, low } \\
\text { shot }\end{array}$ & $\begin{array}{l}\text { sandfarben, } \\
\text { beige/ } \\
\text { Tageslicht }\end{array}$ & $\begin{array}{l}\text { lange, scheinbar } \\
\text { unendliche } \\
\text { TreppeinBergen, } \\
\text { Noé trägt das } \\
\text { Fahrrad }\end{array}$ \\
\hline 44 & $\begin{array}{l}50.04- \\
52.02\end{array}$ & $\begin{array}{l}\text { Fußweg nach } \\
\text { Hause durch } \\
\text { Manchay }\end{array}$ & F, Noé & $\begin{array}{l}\text { F erzähltvon } \\
\text { traurigen Seelen } \\
\text { und ihrem } \\
\text { verstorbenen } \\
\text { Bruder, beide } \\
\text { laufen dicht an } \\
\text { den Mauern } \\
\text { entlang, Noé }\end{array}$ & $\begin{array}{l}\text { Schritte und } \\
\text { Fahrrad im } \\
\text { Land, Geräusche } \\
\text { von } \\
\text { Bauarbeiten, } \\
\text { Gurren der } \\
\text { Tauben }\end{array}$ & $\begin{array}{l}\text { 1. medium long } \\
\text { shot ( } F \text {, Noé), } \\
\text { Kamera bereits auf } \\
\text { Position, beide } \\
\text { treten ins Bild, } \\
\text { normal camera } \\
\text { height }\end{array}$ & $\begin{array}{l}\text { sandfarben/ } \\
\text { Tageslicht }\end{array}$ & $\begin{array}{l}\text { Armenviertel sehr } \\
\text { provisorisch, nicht } \\
\text { fertiggestellte } \\
\text { Häuser, halbhohe } \\
\text { Steinmauern, } \\
\text { Pappe als } \\
\text { Trennwände oder } \\
\text { "Weg", gespannte }\end{array}$ \\
\hline
\end{tabular}




\begin{tabular}{|c|c|c|c|c|c|c|c|c|c|}
\hline & & & & $\begin{array}{l}\text { begleitet } F \text {, sie } \\
\text { rennt das letzte } \\
\text { Stück alleine (aber } \\
\text { bedankt sich nicht } \\
\text { einmal), sie lächelt } \\
\text { Noé hinterher }\end{array}$ & & & $\begin{array}{l}\text { 2.close-up (F, Noé), } \\
\text { Nebenherfahrt, } \\
\text { abwechselnd Fund } \\
\text { Noé imBild } \\
\text { 3. Schwenkauf } F, F \\
\text { entfernt sich von } \\
\text { der Kamera bis } \\
\text { medium long shot, } \\
\text { normal camera } \\
\text { height } \\
\text { 4. closeshot(Noé), } \\
\text { normal camera } \\
\text { height } \\
\text { 5. medium long } \\
\text { shot (F), normal } \\
\text { camera height } \\
\text { 6. close-up (f, im } \\
\text { Hintergrundfährt } \\
\text { Noé weg), Schwenk } \\
\text { mit F's } \\
\text { Kopfbewegung }\end{array}$ & & $\begin{array}{l}\text { Wäscheleinen mit } \\
\text { Kleidern darauf }\end{array}$ \\
\hline 45 & $\begin{array}{l}52.03- \\
52.43\end{array}$ & B. & $\begin{array}{l}\text { Tío, F, Kinder } \\
\text { Kinder tragen } \\
\text { Badekleider, ein } \\
\text { Kind trägt Helm in } \\
\text { Tarnfarben, } \\
\text { Schwimmreifen } \\
\text { und anderes } \\
\text { Spielzeug im Loch } \\
\text { Tío mit Schaufel } \\
\text { F: offene Haare, } \\
\text { Taubenkette gut } \\
\text { sichtbar }\end{array}$ & $\begin{array}{l}\text { F sieht die Grube, } \\
\text { ist erst schockiert, } \\
\text { Kinder spielen im } \\
\text { Pool }\end{array}$ & Taubenkette & $\begin{array}{l}\text { Gitarren- } \\
\text { musik, Kinder } \\
\text { im Wasser, Off: } \\
\text { Gesang F }\end{array}$ & $\begin{array}{l}\text { 1. medium long } \\
\text { shot (tío), high } \\
\text { shot/subj. Kamera } \\
\text { (Persp. F) } \\
\text { 2. close-up (F), } \\
\text { Vorwegfahrt } \\
\text { 3. full shot (tío), } \\
\text { Kamera bewegt } \\
\text { sich auftíozu, subj. } \\
\text { Kamera (Persp. F) } \\
\text { 4. close-up (F), } \\
\text { Vorwegfahrt } \\
\text { 5. close shot } \\
\text { (Grube), Kamera } \\
\text { bewegt sich auf } \\
\text { Grubezu, high }\end{array}$ & $\begin{array}{l}\text { sandfarben/ } \\
\text { Tageslicht }\end{array}$ & $\begin{array}{l}\text { Loch mit Leiter im } \\
\text { Hof, man sieht } \\
\text { nicht, wasin dem } \\
\text { Loch ist, tío steht } \\
\text { daneben, } \\
\text { erst als F sich } \\
\text { näher, sieht man } \\
\text { die Kinder, } \\
\text { Sonnenuntergang } \\
\text { hinter F }\end{array}$ \\
\hline
\end{tabular}




\begin{tabular}{|c|c|c|c|c|c|c|c|c|c|}
\hline & & & & & & & $\begin{array}{l}\text { shot, subj. Kamera } \\
\text { (Persp. F) } \\
\text { 6. close-up (F), } \\
\text { subj. Kamera } \\
\text { (Persp. Kinder)/ } \\
\text { Kamera auf } \\
\text { Brusthöhe, leicht } \\
\text { von unten }\end{array}$ & & \\
\hline 46 & $\begin{array}{l}52.44- \\
53.27\end{array}$ & A. & F, Leichnam & $\begin{array}{l}\text { F singt, F streicht } \\
\text { ihrer Mutter durch } \\
\text { die Haare, eine } \\
\text { Haarsträhne von } \\
\text { ihrer Mutter löst } \\
\text { sich, F lässt die } \\
\text { Strähnevom Wind } \\
\text { mitnehmen }\end{array}$ & $\begin{array}{l}\text { Lied: } \\
\text { Übersetzung } \\
\text { aus Quechua: } \\
\text { A ver si la } \\
\text { gente no te } \\
\text { reconoce } \\
\text { cuando } \\
\text { lleguemos al } \\
\text { pueblo. Te } \\
\text { llevaré cargado } \\
\text { como un bebé. } \\
\text { Mi papá ya no } \\
\text { va a estar } \\
\text { solito con los } \\
\text { gusanos... } \\
\text { Mumie }\end{array}$ & $\begin{array}{l}\text { On: Gesang F, } \\
\text { Gurren der } \\
\text { Tauben, } \\
\text { Gitarrenmusik }\end{array}$ & $\begin{array}{l}\text { 1. extreme close-up } \\
\text { (Perpetua's Haare), } \\
\text { Schwenk über } \\
\text { Kopfkissen, close- } \\
\text { up (F), high shot } \\
\text { 2. close-up (F's Arm } \\
\text { mit Haarsträhne), } \\
\text { normal camera } \\
\text { height }\end{array}$ & $\begin{array}{l}\text { türkis, weiß/ } \\
\text { Tageslicht von } \\
\text { draußen }\end{array}$ & $\begin{array}{l}\text { lange, schwarz- } \\
\text { graue } \\
\text { Haarsträhnen } \\
\text { liegen auf dem } \\
\text { Kopfkissen (mit } \\
\text { Blumen und } \\
\text { Schriftzug „nome } \\
\text { olvides" bestickt), } \\
\text { F liegt nah bei } \\
\text { neben ihrer toten } \\
\text { Mutter, } \\
\text { Arm (ohne } \\
\text { Körper) am } \\
\text { Fenster, Blick auf } \\
\text { den Hof wird } \\
\text { durch blauen } \\
\text { Vorhang } \\
\text { verringert }\end{array}$ \\
\hline 47 & $\begin{array}{l}53.28- \\
53.36\end{array}$ & D. & $\begin{array}{l}\text { F } \\
\text { Uniform, Haareim } \\
\text { Zopf }\end{array}$ & F wartet & & Grillen & $\begin{array}{l}\text { 1. medium shot }(F) \text {, } \\
\text { normal camera } \\
\text { height, v. hinten, } \\
\text { statische Kamera }\end{array}$ & $\begin{array}{l}\text { blau, } \\
\text { sandfarben, } \\
\text { steingrau/ } \\
\text { schummriges } \\
\text { Licht von } \\
\text { Lampe links }\end{array}$ & $\begin{array}{l}\text { F sitzt starr auf } \\
\text { dem Bettmit } \\
\text { Gesicht Richtung } \\
\text { Fenster }\end{array}$ \\
\hline 48 & $\begin{array}{l}53.37- \\
54.35\end{array}$ & $\begin{array}{l}\text { Weg durch } \\
\text { das } \\
\text { Herrenhaus }\end{array}$ & $\mathrm{F}$ & $\begin{array}{l}\text { Fgehtlangsam } \\
\text { durch das } \\
\text { Herrenhaus } \\
\text { (angespannter }\end{array}$ & $\begin{array}{l}\text { Lied (Off): } \\
\text { Dicen en mi } \\
\text { pueblo quelos } \\
\text { músicos hacen }\end{array}$ & $\begin{array}{l}\text { Off: Gesang F } \rightarrow \\
\text { F singt im Kopf }\end{array}$ & $\begin{array}{l}\text { 1. close-up }(F) \text {, } \\
\text { Vorwegfahrt, } \\
\text { normal camera } \\
\text { height }\end{array}$ & $\begin{array}{l}\text { senfgelb/- } \\
\text { grün, } \\
\text { schummriges, } \\
\text { stellenweises }\end{array}$ & $\begin{array}{l}\text { F schleicht } \\
\text { langsamdurch die } \\
\text { halbdunklen } \\
\text { Flure, an }\end{array}$ \\
\hline
\end{tabular}




\begin{tabular}{|c|c|c|c|c|c|c|c|c|c|}
\hline & & & & $\begin{array}{l}\text { Blick, inGedanken } \\
\text { versunken, } \\
\text { aufgeregt, Tränen } \\
\text { im Auge) }\end{array}$ & $\begin{array}{l}\text { un contrato } \\
\text { con una sirena. } \\
\text { Si quieren } \\
\text { saber cuánto } \\
\text { durará, durará } \\
\text { el contrato con } \\
\text { esa sirena. De } \\
\text { un campo } \\
\text { oscuro tienen } \\
\text { que coger un } \\
\text { puñado de } \\
\text { quinua para la } \\
\text { sirena. Ya si la } \\
\text { sirena sigue de } \\
\text { contando, dice } \\
\text { la sirena que } \\
\text { cada grano } \\
\text { significa un } \\
\text { año. Cuandola } \\
\text { sirena termine } \\
\text { de contar, selo } \\
\text { lleva el } \\
\text { hombre y le } \\
\text { suelta al mar. } \\
\text { Labyrinth } \\
\end{array}$ & & & $\begin{array}{l}\text { Licht von } \\
\text { Lampen (F } \\
\text { Gesicht oft im } \\
\text { Schatten) }\end{array}$ & $\begin{array}{l}\text { Gemälden vorbei, } \\
\text { engan der Wand } \\
\text { aber ohne sie zu } \\
\text { berühren (wieim } \\
\text { Horrorfilm, wenn } \\
\text { man nicht weiß, } \\
\text { was am Ende des } \\
\text { Flures auf einen } \\
\text { wartet) }\end{array}$ \\
\hline 49 & $\begin{array}{l}54.36- \\
55.17\end{array}$ & $\mathrm{~F}$. & $\begin{array}{l}\text { F, Aída } \\
\text { Aída: dünner } \\
\text { Pullover, Brille }\end{array}$ & $\begin{array}{l}\text { F singt atemlos } \\
\text { das Lied der } \\
\text { Sirena, Aída hört } \\
\text { zu und zählt die } \\
\text { erste Perle in die } \\
\text { andere Schale der } \\
\text { Waage ab }\end{array}$ & $\begin{array}{l}\text { Lied (On): Pero } \\
\text { mi madre dice, } \\
\text { dice, dice que } \\
\text { la quinua difícil } \\
\text { de contar es y } \\
\text { la sirena se } \\
\text { cansa de } \\
\text { contar. Ya esel } \\
\text { hombre para } \\
\text { siempre, ya se } \\
\text { queda con él } \\
\text { don. }\end{array}$ & $\begin{array}{l}\text { On: Gesang F, } \\
\text { Tik-Tak der Uhr, } \\
\text { Perle }\end{array}$ & $\begin{array}{l}\text { 1. close-up (Aída, v. } \\
\text { hinten), normal } \\
\text { camera height } \\
\text { 2. close-up (F), } \\
\text { normal camera } \\
\text { height } \\
\rightarrow \text { Wdh. } 1+2 \\
\text { 3. close-up (Waage } \\
\text { mit Perlen), high } \\
\text { shot }\end{array}$ & $\begin{array}{l}\text { beige, } \\
\text { steingrau, } \\
\text { schummriges } \\
\text { Licht von } \\
\text { Lampen }\end{array}$ & $\begin{array}{l}\text { F Träne im Auge } \\
\text { (leidend), Aída mit } \\
\text { grimmigem, leicht } \\
\text { verwirtem Blick } \\
\text { dreht den Kopf in } \\
\text { F's Richtung } \\
\text { (etwas Böses im } \\
\text { Blick) }\end{array}$ \\
\hline
\end{tabular}




\begin{tabular}{|c|c|c|c|c|c|c|c|c|c|}
\hline 50 & $\begin{array}{l}55.18- \\
56.30\end{array}$ & C. & F, Noé & $\begin{array}{l}\text { NoéundFreden } \\
\text { überPflanzen } \\
\text { (Noé macht damit } \\
\text { Anspielungen auf } \\
\text { F) }\end{array}$ & Pflanzen & $\begin{array}{l}\text { Vogelge- } \\
\text { zwitscher, } \\
\text { Geräusche von } \\
\text { außerhalb des } \\
\text { Herren- } \\
\text { hauses }\end{array}$ & $\begin{array}{l}\text { 1. close-up (F v. } \\
\text { hinten), } \\
\text { Hinterherfahrt } \\
\text { 2. close shot (F, } \\
\text { Noé), normal } \\
\text { camera height }\end{array}$ & $\begin{array}{l}\text { grün, türkis/ } \\
\text { Tageslicht }\end{array}$ & $\begin{array}{l}\text { Fund Noéhinter } \\
\text { Mauer mit } \\
\text { Pflanzen, F sieht } \\
\text { man kaum hinter } \\
\text { den Pflanzen }\end{array}$ \\
\hline 51 & $\begin{array}{l}56.31- \\
56.52\end{array}$ & $\begin{array}{l}\text { C., } \\
\text { Hinterhof }\end{array}$ & F, Noé & $\begin{array}{l}\text { Noé verbrennt das } \\
\text { Klavier, F schaut } \\
\text { zu }\end{array}$ & & $\begin{array}{l}\text { Knacken des } \\
\text { Feuers }\end{array}$ & $\begin{array}{l}\text { 1. medium long } \\
\text { shot ( } F \text {, Noé, } \\
\text { Feuer), normal } \\
\text { camera height, } \\
\text { statische Kamera }\end{array}$ & $\begin{array}{l}\text { sandfarben, } \\
\text { braunrot/ } \\
\text { Dämmerung, } \\
\text { Feuer }\end{array}$ & $\begin{array}{l}\text { brennendes } \\
\text { Klavier rechts, F } \\
\text { an } \\
\text { Ziegelsteinmauer } \\
\text { gelehnt } \\
\text { (entspannte } \\
\text { Haltung), an der } \\
\text { Wand Holzreste }\end{array}$ \\
\hline 52 & $\begin{array}{l}56.53- \\
57.28\end{array}$ & B. & $\begin{array}{l}\text { Familie (ohne F, } \\
\text { passive Präsenz } \\
\text { von F, sie } \\
\text { beobachtet) } \\
\text { farbenfrohe } \\
\text { Badebekleidung, } \\
\text { Máxima (pink) } \\
\text { sticht hervor, } \\
\text { trotz der } \\
\text { Entfernung }\end{array}$ & $\begin{array}{l}\text { Familie spielt am } \\
\text { Pool }\end{array}$ & & $\begin{array}{l}\text { fröhliche Musik, } \\
\text { Kinder- } \\
\text { geschrei, } \\
\text { Hundebellen }\end{array}$ & $\begin{array}{l}\text { 1. long shot } \\
\text { (Familie), high } \\
\text { shot/subj. Kamera } \\
\text { (Persp. F) }\end{array}$ & $\begin{array}{l}\text { sandfarben, } \\
\text { beige, bunte } \\
\text { Farbflecken/ } \\
\text { Tageslicht } \\
\text { (bewölkt) }\end{array}$ & $\begin{array}{l}\text { Familie sitzt um } \\
\text { den Pool herum, } \\
\text { drum herum } \\
\text { Armenviertel } \\
\text { (siehtauswieeine } \\
\text { reine Baustelle, } \\
\text { nichts ist } \\
\text { fertiggestellt), } \\
\text { Berge im } \\
\text { Hintergrund }\end{array}$ \\
\hline 53 & $\begin{array}{l}57.29- \\
57.40\end{array}$ & A. & $\begin{array}{l}\text { F } \\
\text { Haare im Zopf }\end{array}$ & $\begin{array}{l}\text { F beobachtet } \\
\text { Familie von ihrem } \\
\text { Fenster aus }\end{array}$ & & $\begin{array}{l}\text { fröhliche Musik } \\
\text { vom Hof, } \\
\text { Gurren der } \\
\text { Tauben }\end{array}$ & $\begin{array}{l}\text { 1. close shot (Fv. } \\
\text { hinten durch den } \\
\text { Vorhang), normal } \\
\text { camera height }\end{array}$ & blau & $\begin{array}{l}\text { F steht am } \\
\text { Fenster, der blaue } \\
\text { Vorhang legt sich } \\
\text { über sie (man } \\
\text { sieht sie durch } \\
\text { den Vorhang) }\end{array}$ \\
\hline 54 & $\begin{array}{l}57.41- \\
58.17\end{array}$ & $\begin{array}{l}\text { Weg durch } \\
\text { das } \\
\text { Herrenhaus }\end{array}$ & $\begin{array}{l}\text { F } \\
\text { Uniform }\end{array}$ & $\begin{array}{l}\text { Fschaltet Lichter } \\
\text { im Herrenhausaus }\end{array}$ & & $\begin{array}{l}\text { Off: Gesang F } \rightarrow \\
\text { im Kopf, } \\
\text { Schritteim Haus }\end{array}$ & $\begin{array}{l}\text { 1. full shot }(F) \text {, } \\
\text { Kamera bereits auf } \\
\text { Position, F geht } \\
\text { entlang des Bildes }\end{array}$ & $\begin{array}{l}\text { steingrau, } \\
\text { braun/ } \\
\text { schummriges } \\
\text { Licht }\end{array}$ & $\begin{array}{l}\text { dunkles } \\
\text { Herrenhaus, nur } \\
\text { Licht wo Lampen } \\
\text { stehen, Labyrinth }\end{array}$ \\
\hline
\end{tabular}




\begin{tabular}{|c|c|c|c|c|c|c|c|c|c|}
\hline & & & & & & & $\begin{array}{l}\text { 2. full shot }(\mathrm{F}) \text {, } \\
\text { Kamera bereits auf } \\
\text { Position, F geht } \\
\text { entlang des Bildes } \\
\text { 3. full shot (F), } \\
\text { Kamera bereits auf } \\
\text { Position, F geht } \\
\text { entlang des Bildes }\end{array}$ & $\begin{array}{l}\text { (stellenweise) } \\
\text { von Lampen }\end{array}$ & \\
\hline 55 & $\begin{array}{l}58.18- \\
59.25\end{array}$ & A. & $\begin{array}{l}\text { F } \\
\text { Nachthemd }\end{array}$ & $\begin{array}{l}\text { F wäscht sich das } \\
\text { Gesicht und } \\
\text { schneidet eine } \\
\text { Wurzel von ihrer } \\
\text { Kartoffel ab }\end{array}$ & Kartoffel & $\begin{array}{l}\text { Off: Gesang } \mathrm{F} \rightarrow \\
\text { im Kopf, } \\
\text { Schneiden der } \\
\text { Wurzel }\end{array}$ & $\begin{array}{l}\text { 1. close shot } \\
\text { (Spiegelbild F), über } \\
\text { F's Schulter gefilmt, } \\
\text { normal camera } \\
\text { height, } \\
\text { Handkamera } \\
\text { 2. close shot, } \\
\text { Schwenk, Kamera } \\
\text { verfolgtF, verharrt } \\
\text { auf ihrem Gesicht } \\
\text { (close-up) } \\
\text { 3. extreme close-up } \\
\text { (F'sFüße. Wurzel), } \\
\text { normal camera } \\
\text { height } \\
\text { 4. close-up (F's } \\
\text { Gesicht), Kamera } \\
\text { bewegt sich mitbis } \\
\text { medium shot (F), } \\
\text { high shot }\end{array}$ & $\begin{array}{l}\text { hellblau, } \\
\text { sandfarben/ } \\
\text { Tageslicht von } \\
\text { draußen }\end{array}$ & $\begin{array}{l}\text { Hintergrund: } \\
\text { Leichnam auf Bett } \\
\text { Vordergrund: F } \\
\text { und Spiegelbild, } \\
\text { Zahnbürsten und } \\
\text { Kämme auf der } \\
\text { Kommodevorihr, } \\
\text { legt sich auf das } \\
\text { Bett }\end{array}$ \\
\hline \multicolumn{10}{|c|}{3 Sekunden Schwarzblende } \\
\hline 56 & $\begin{array}{r}59.29- \\
1.00 .58\end{array}$ & C. & F, Aída & $\begin{array}{l}\text { Aída gießt } \\
\text { Pflanzen, F singt } \\
\text { für sie, Aída will } \\
\text { dasLied derSirena } \\
\text { hören, Aída } \\
\text { entdeckt } \\
\text { Puppe im Beet: }\end{array}$ & Erde & $\begin{array}{l}\text { On: GesangF } \\
\text { und Summen } \\
\text { von Aída, } \\
\text { Wasser, } \\
\text { Vogelge- } \\
\text { zwitscher }\end{array}$ & $\begin{array}{l}\text { 1. medium full shot } \\
\text { ( } F, \text { Aída, ohne } \\
\text { Füße), normal } \\
\text { camera height } \\
\text { 2. close-up (Gesicht } \\
\text { Aída im Profil, F's } \\
\text { Nase/ Mund), }\end{array}$ & $\begin{array}{l}\text { mintgrün, } \\
\text { steingrau/ } \\
\text { Tageslicht }\end{array}$ & $\begin{array}{l}\text { Frauen stehen } \\
\text { nebeneinander } \\
\text { ohne Blickkontakt } \\
\text { vor dem Beet }\end{array}$ \\
\hline
\end{tabular}




\begin{tabular}{|c|c|c|c|c|c|c|c|c|}
\hline & & & & $\begin{array}{l}\text { "medijeron quesi } \\
\text { la enterraba, luego } \\
\text { la tierra se la } \\
\text { llevaba ynuncala } \\
\text { encontrabas... } \\
\text { mentirosos" ( } \rightarrow \\
\text { Auswirkung aufF's } \\
\text { spätere } \\
\text { Entscheidung?) }\end{array}$ & & $\begin{array}{l}\text { normal camera } \\
\text { height, Aída } \\
\text { bewegt sich aus } \\
\text { dem Bild und legt } \\
\text { F's Gesicht frei) } \\
\text { 3. close shot } \\
\text { (Puppe), high shot } \\
\text { 4. close-up } \\
\text { (Gesichter), normal } \\
\text { camera height }\end{array}$ & & \\
\hline 57 & $\begin{array}{l}1.00 .59- \\
1.01 .03\end{array}$ & $\mathrm{~F}$. & & $\begin{array}{l}\text { Perlen in der } \\
\text { Waage }\end{array}$ & & $\begin{array}{l}\text { 1. extreme close-up } \\
\text { (Waage mit } \\
\text { Perlen), extreme } \\
\text { high shot }\end{array}$ & weiß & Waage im Fokus \\
\hline 58 & $\begin{array}{l}1.01 .04- \\
1.01 .28\end{array}$ & C. & $\begin{array}{l}\text { F, Klavierträger } \\
\text { Männer alle } \\
\text { schwarze Hosen } \\
\text { mit Hosenträgern } \\
\text { und hellblauem T- } \\
\text { Shirt }\end{array}$ & $\begin{array}{l}\text { F läuft rückwärts } \\
\text { vor den } \\
\text { Klavierträgern her, } \\
\text { diese tragen es } \\
\text { angestrengt }\end{array}$ & $\begin{array}{l}\text { Gitarren- } \\
\text { musik, Schritte }\end{array}$ & $\begin{array}{l}\text { 1. medium shot (F), } \\
\text { Hinterherfahrt, F } \\
\text { läuft rückwärts/ } \\
\text { subj. Kamera } \\
\text { (Persp. Männer) } \\
\text { 2. medium long } \\
\text { shot (Männer, } \\
\text { Klavier), } \\
\text { Vorwegfahrt/ subj. } \\
\text { Kamera(Persp. F) }\end{array}$ & $\begin{array}{l}\text { hellblau, } \\
\text { schwarz, grün, } \\
\text { steingrau }\end{array}$ & $\begin{array}{l}\text { Weg durch den } \\
\text { Garten }\end{array}$ \\
\hline 59 & $\begin{array}{l}1.01 .29- \\
1.02 .32\end{array}$ & $\begin{array}{l}\text { Klavier- } \\
\text { zimmer im } \\
\text { Herrenhaus }\end{array}$ & F, Aída & $\begin{array}{l}\text { Fzieht Tuch vom } \\
\text { Klavierab, Aída } \\
\text { sieht das neue } \\
\text { Klavier und geht }\end{array}$ & $\begin{array}{l}\text { Gitarren- } \\
\text { musik (einzelne } \\
\text { Akkorde) }\end{array}$ & $\begin{array}{l}\text { 1. medium long } \\
\text { shot ( } F, \text { Klavier), } \\
\text { statische Kamera, F } \\
\text { geht aus dem Bild } \\
\text { 2. medium shot } \\
\text { (Aída), statische } \\
\text { Kamera bereits auf } \\
\text { Position, Aída geht } \\
\text { entlang des Bildes }\end{array}$ & $\begin{array}{l}\text { beige, } \\
\text { mintgrün/ } \\
\text { Licht von } \\
\text { Lampe an der } \\
\text { Decke }\end{array}$ & $\begin{array}{l}\text { Klavier und } \\
\text { Hocker mittig im } \\
\text { Raum } \\
\text { (Durchgangs- } \\
\text { zimmer, linksund } \\
\text { rechts dunkle } \\
\text { Flure), auf einem } \\
\text { Orientteppich, an } \\
\text { der Wand großer } \\
\text { antiker Spiegel } \\
\text { mit schwerem } \\
\text { Holzrahmen }\end{array}$ \\
\hline
\end{tabular}




\begin{tabular}{|c|c|c|c|c|c|c|c|c|c|}
\hline & & & & & & & & & $\begin{array}{l}\text { Blick von } \\
\text { Klavierhocker in } \\
\text { linkes Zimmer, } \\
\text { Aída läuft durch, } \\
\text { sieht das Klavier } \\
\text { und geht weiter } \\
\text { (böser Blick) }\end{array}$ \\
\hline 60 & $\begin{array}{l}1.02 .33- \\
1.03 .53\end{array}$ & C. & $\begin{array}{l}\text { F, Noé } \\
\text { F: Uniform, rote } \\
\text { Blume in voller } \\
\text { Blüte im Mund, } \\
\text { ihr Hosenbein } \\
\text { unter dem Rock } \\
\text { rutscht hinunter } \\
\text { alssievorSchreck } \\
\text { dieBonbonsfallen } \\
\text { lässt }\end{array}$ & $\begin{array}{l}F \text { (mit Blume im } \\
\text { Mund) öffnet das } \\
\text { Torfür Noé, laufen } \\
\text { nebeneinander, F } \\
\text { hat ein } \\
\text { zufriedenes } \\
\text { Gesicht, er schenkt } \\
\text { ihr Bonbons, bei } \\
\text { Berührunglässt sie } \\
\text { siefallen und läuft } \\
\text { weg }\end{array}$ & Blume & $\begin{array}{l}\text { Fahrrad, Öffnen } \\
\text { des } \\
\text { Eingangstors, } \\
\text { Stimmen- } \\
\text { gewirr, Vogelge- } \\
\text { zwitscher, } \\
\text { fallende } \\
\text { Bonbons, } \\
\text { schnelle Schritte }\end{array}$ & $\begin{array}{l}\text { 1. close-up (F, } \\
\text { Blume), normal } \\
\text { camera height, } \\
\text { Nebenherfahrt bis } \\
\text { extreme close-up } \\
\text { (F, Blume) } \\
\text { 2. close-up (F, } \\
\text { Noé), } \\
\text { Nebenherfahrt } \\
\text { 3. close-up (F's } \\
\text { Gesicht), normal } \\
\text { camera height } \\
\text { 4. close-up (F's } \\
\text { Hand am Rock), } \\
\text { normal camera } \\
\text { height, Kamera } \\
\text { verfolgt Hand, } \\
\text { close-up (beide } \\
\text { Hände) } \\
\text { 5. close-up (Füße, } \\
\text { Bonbons), high } \\
\text { shot } \\
\text { 6. close-up (F's } \\
\text { Gesicht), normal } \\
\text { camera height } \\
\text { 7. close-up (Noé, } \\
\text { F), normal camera } \\
\text { height }\end{array}$ & $\begin{array}{l}\text { grün, } \\
\text { steingrau, } \\
\text { dunkelrot/ } \\
\text { Tageslicht }\end{array}$ & $\begin{array}{l}\text { Blume bedeckt F's } \\
\text { halbes Gesicht, F } \\
\text { ist wieder an } \\
\text { Steinwand } \\
\text { gedrückt, lässt } \\
\text { Blume fallen kurz } \\
\text { bevor sie Noé } \\
\text { sieht }\end{array}$ \\
\hline
\end{tabular}




\begin{tabular}{|c|c|c|c|c|c|c|c|c|}
\hline & & & & & & $\begin{array}{l}\text { 8. medium long } \\
\text { shot }(F), \text { Fentfernt } \\
\text { sich von Kamera }\end{array}$ & & \\
\hline 61 & $\begin{array}{l}1.03 .54- \\
1.05 .05\end{array}$ & $\begin{array}{l}\text { C., } \\
\text { Hinterhof }\end{array}$ & $\begin{array}{l}\text { F, Noé } \\
\text { F: Jogginghose, T- } \\
\text { Shirt }\end{array}$ & $\begin{array}{l}\text { Noéschaufeltdie } \\
\text { Asche zusammen, } \\
\text { beide diskutieren, } \\
\text { F rechtfertigt sich } \\
\text { (wirkt } \\
\text { vorwurfsvoll) }\end{array}$ & $\begin{array}{l}\text { Rascheln der } \\
\text { Schaufel in der } \\
\text { Asche, Vogelge- } \\
\text { zwitscher }\end{array}$ & $\begin{array}{l}\text { 1. medium long } \\
\text { shot (Noé), normal } \\
\text { camera height, } F \\
\text { tritt ins Bild, } \\
\text { statische Kamera } \\
\text { 2. close shot(Noé), } \\
\text { Kamera bewegt } \\
\text { sich mit ihm, } \\
\text { normal camera } \\
\text { height } \\
\text { 3. close shot (F), } \\
\text { Kamera bewegt } \\
\text { sich mit ihr mit, } \\
\text { normal camera } \\
\text { height } \rightarrow \\
\text { mehrmaligeWdh.2 } \\
+3 \\
4 . \text { full shot (F), } \\
\text { normal camera } \\
\text { height, F entfernt } \\
\text { sich von Kamera }\end{array}$ & $\begin{array}{l}\text { steingrau, } \\
\text { blau/ } \\
\text { Dämmerung } \\
\text { abernoch hell }\end{array}$ & $\begin{array}{l}\text { Noé bewegt sich } \\
\text { um den } \\
\text { Aschehaufen } \\
\text { herum, } F \text { kommt } \\
\text { durch Durchgang } \\
\text { in der Mauer zu } \\
\text { inm, geht etwas } \\
\text { aufgebracht aber } \\
\text { trotzdem steif hin } \\
\text { und her }\end{array}$ \\
\hline 62 & $\begin{array}{l}1.05 .06- \\
1.07 .00\end{array}$ & Manchay & $\begin{array}{l}\text { Hochzeits- } \\
\text { gesellschaft, Tío, } \\
\text { Tante, F } \\
\text { Anzüge und } \\
\text { Brautkleider, F } \\
\text { und Familie in } \\
\text { maskuliner } \\
\text { Uniform }\end{array}$ & $\begin{array}{l}\text { Massenhochzeit, } \\
\text { Geschenkevergabe } \\
\text { Fwird ohnmächtig }\end{array}$ & $\begin{array}{l}\text { fröhliche Musik, } \\
\text { Applaus, Wind }\end{array}$ & $\begin{array}{l}\text { 1. long shot } \\
\text { (Verlobte), } \\
\text { statische Kamera } \\
\text { 2. Kamera Schwenk } \\
\text { über ganze } \\
\text { Gesellschaft, } \\
\text { gleichmäßiges (fast } \\
\text { schon } \\
\text { mechanisches) } \\
\text { Gleiten (medium } \\
\text { long shot) }\end{array}$ & $\begin{array}{l}\text { sandfarben, } \\
\text { weiß, schwarz, } \\
\text { rosa, blau, rot/ } \\
\text { Tageslicht, } \\
\text { klarer Himmel }\end{array}$ & $\begin{array}{l}\text { Anden im } \\
\text { Hintergrund, } \\
\text { große, weite, } \\
\text { leere Fläche mit } \\
\text { Sand, Stühle mit } \\
\text { kitschiger } \\
\text { Hochzeitsdeko, im } \\
\text { Dorf roter } \\
\text { Teppich, kitschige } \\
\text { Deko, Podestmit } \\
\text { Geschenken }\end{array}$ \\
\hline
\end{tabular}




\begin{tabular}{|c|c|c|c|c|c|c|c|c|c|}
\hline 63 & $\begin{array}{l}1.07 .01- \\
1.07 .25\end{array}$ & $\begin{array}{l}\text { Manchay } \\
\text { (Abseits der } \\
\text { Feier) }\end{array}$ & F， Tío & $\begin{array}{l}\text { Onkel kümmert } \\
\text { sich um } \mathrm{F} \text {, die } \\
\text { gekrümmt am } \\
\text { Boden hockt und } \\
\text { sich den Bauch vor } \\
\text { Schmerzen reibt }\end{array}$ & & $\begin{array}{l}\text { fröhliche Musik } \\
\text { von der Feier }\end{array}$ & $\begin{array}{l}\text { 1. medium long } \\
\text { shot ( } F, \text { tío), } \\
\text { statische Kamera }\end{array}$ & $\begin{array}{l}\text { beige, } \\
\text { schwarz, } \\
\text { sandfarben/ } \\
\text { Tageslicht }\end{array}$ & $\begin{array}{l}\text { Holzzaun, } \\
\text { dahinter Gebüsch } \\
\text { mit rosa Blüten, } \\
\text { im Hintergrund } \\
\text { Armenviertel in } \\
\text { Bergen }\end{array}$ \\
\hline 64 & $\begin{array}{l}1.07 .26- \\
1.07 .56\end{array}$ & $\begin{array}{l}\text { Warteflur } \\
\text { im } \\
\text { Krankenhaus }\end{array}$ & $\begin{array}{l}\text { F, Verwandter, } \\
\text { schwangere } \\
\text { Frauen } \\
\text { Alltagskleider, F } \\
\text { und Verwandter } \\
\text { inUniform (ohne } \\
\text { Krawatte) }\end{array}$ & $\begin{array}{l}\text { F und Verwandter } \\
\text { warten im } \\
\text { Krankenhausflur, } \\
\text { schwangere } \\
\text { Frauen sprechen } \\
\text { über Kleiderpreise, } \\
\text { F beobachtet die } \\
\text { großen Bäuche } \\
\text { von dem Frauen } \\
\text { und reibt sich } \\
\text { ihren }\end{array}$ & & $\begin{array}{l}\text { fröhliche Musik } \\
\text { aus den } \\
\text { Kopfhörern des } \\
\text { Verwandten }\end{array}$ & $\begin{array}{l}\text { 1. mediumshot(F, } \\
\text { Verwandter, } \\
\text { Frauen), normal } \\
\text { camera height, } \\
\text { statische Kamera }\end{array}$ & $\begin{array}{l}\text { beige/ } \\
\text { Tageslicht }\end{array}$ & $\begin{array}{l}\text { kahler, wenig } \\
\text { einladender } \\
\text { Krankenhausflur } \\
\text { mit Holzbänken } \\
\text { an der Wand } \\
\text { (Holz, Beton) }\end{array}$ \\
\hline 65 & $\begin{array}{l}1.07 .57- \\
1.08 .43\end{array}$ & $\begin{array}{l}\text { Kranken- } \\
\text { haus, Be- } \\
\text { sprechungs- } \\
\text { zimmer }\end{array}$ & $\begin{array}{l}\text { F, Arzt } \\
\text { Arzt: Ärztekittel }\end{array}$ & $\begin{array}{l}\text { Arzt weist F ab, } \\
\text { weil sie die } \\
\text { Papiere nicht } \\
\text { dabei hat, redet } \\
\text { dabei etwas } \\
\text { abschätzig, F hört } \\
\text { zu (mit } \\
\text { vorwurfsvollem, } \\
\text { fast schon bösen } \\
\text { Blick) }\end{array}$ & & $\begin{array}{l}\text { Stimmen aus } \\
\text { dem Flur }\end{array}$ & $\begin{array}{l}\text { 1. close shot(Arzt), } \\
\text { normal camera } \\
\text { height, Schuss } \\
\text { 2. close shot }(F) \text {, } \\
\text { normal camera } \\
\text { height, } \\
\text { Gegenschuss } \\
\rightarrow \text { mehrmalige } \\
\text { Wdh } 1+2\end{array}$ & $\begin{array}{l}\text { beige, weiß/ } \\
\text { Tageslicht von } \\
\text { Fenstern }\end{array}$ & $\begin{array}{l}\text { Schreibtisch mit } \\
\text { hohen Stapeln } \\
\text { von Papieren, } \\
\text { hinter dem Arzt } \\
\text { Ablage aus Holz, } \\
\text { hinter F } \\
\text { Waschbecken und } \\
\text { Ablage aus } \\
\text { weißen Fließen, } \\
\text { Ärztewerkzeuge }\end{array}$ \\
\hline 66 & $\begin{array}{l}1.08 .44- \\
1.10 .06\end{array}$ & B. & $\begin{array}{l}\text { F, Tío } \\
\text { Alltagskleidung }\end{array}$ & $\begin{array}{l}\text { F trägt } \\
\text { Wassereimer für } \\
\text { Tauben, Hund } \\
\text { verfolgtsie, F } \\
\text { opfert kranke } \\
\text { Taube }\end{array}$ & Tauben & $\begin{array}{l}\text { hechelnder/ } \\
\text { bellender Hund, } \\
\text { Schritte im } \\
\text { Sand, Gurren } \\
\text { der Tauben, } \\
\text { Flattern der } \\
\text { Taube }\end{array}$ & $\begin{array}{l}\text { 1. close-up (F's } \\
\text { Geschlecht, Hände } \\
\text { und Hund), } \\
\text { Nebenherfahrt } \\
\text { 2. medium long } \\
\text { shot(F,tío, Hund), } \\
\text { normal camera } \\
\text { height }\end{array}$ & $\begin{array}{l}\text { beige, weiß/ } \\
\text { Tageslicht }\end{array}$ & $\begin{array}{l}\text { F geht mit } \\
\text { schweren } \\
\text { Wassereimern } \\
\text { über den Hof, } \\
\text { Treppe hoch auf } \\
\text { das Dach (Stäbe } \\
\text { für weiteres } \\
\text { Geschoss ragen } \\
\text { aus dem Beton, }\end{array}$ \\
\hline
\end{tabular}




\begin{tabular}{|c|c|c|c|c|c|c|c|c|c|}
\hline & & & & & & & $\begin{array}{l}\text { 3. full shot (F, } \\
\text { Hund), F nähert } \\
\text { sich derKamera bis } \\
\text { close shot, Kamera } \\
\text { verfolgt F, ihre } \\
\text { Handlungen, die } \\
\text { Taube und endet } \\
\text { auf close shot } \\
\text { (Taube, Hund) }\end{array}$ & & $\begin{array}{l}\text { darauf stecken } \\
\text { leere } \\
\text { Plastikflaschen } \\
\text { (Schutz?), tío } \\
\text { repariert die } \\
\text { Hochzeitstreppe, } \\
\text { im Hintergrund } \\
\text { Berge, großer } \\
\text { Käfig mit Tauben } \\
\text { auf dem Dach }\end{array}$ \\
\hline 67 & $\begin{array}{l}1.10 .07- \\
1.10 .23\end{array}$ & F. & F (Finger) & F zähltPerlen & & Tik-Tak der Uhr & $\begin{array}{l}\text { 1. extreme close-up } \\
\text { (Waage mit Perlen, } \\
\text { F's Finger), extreme } \\
\text { high shot }\end{array}$ & weiß/ hell & $\begin{array}{l}\text { Fokus auf Waage, } \\
\text { bis auf eine liegen } \\
\text { alleschon auff's } \\
\text { Seite, zählender } \\
\text { Finger }\end{array}$ \\
\hline 68 & $\begin{array}{l}1.10 .24- \\
1.12 .36\end{array}$ & $\begin{array}{l}\text { Ankleide- } \\
\text { zimmer, } \\
\text { Konzerthaus }\end{array}$ & $\begin{array}{l}\text { F, Aída, } \\
\text { Angestellte } \\
\text { Konzerthaus } \\
\text { Aída: schicke } \\
\text { Kleidung, Fin } \\
\text { ihrer grünlichen } \\
\text { Uniform }\end{array}$ & $\begin{array}{l}\text { F föhnt Aída die } \\
\text { Haare, Aída } \\
\text { schminkt sich und } \\
\text { beobachtet } F \text { mit } \\
\text { bösem Blick durch } \\
\text { den Spiegel, } F \\
\text { wartet und nimmt } \\
\text { Blumen für Aída } \\
\text { an, sie hört ihr } \\
\text { Lied (gespielt von } \\
\text { Aída) undfolgtder } \\
\text { Musik zur Bühne, } \\
\text { lächelt am Ende }\end{array}$ & Labyrinth & $\begin{array}{l}\text { Fön, Aída: } \\
\text { immerlauter } \\
\text { werdende } \\
\text { Klaviermusik } \\
\text { (Lied: Sirena) }\end{array}$ & $\begin{array}{l}\text { 1. medium shot } \\
\text { (Aída, F), normal } \\
\text { camera height } \\
\text { (gefilmt durch } \\
\text { einen Spiegel) } \\
\text { 2. close shot ( } F \text {, } \\
\text { Spiegel hinter F, } \\
\text { Blumen, } \\
\text { Angestellte), } \\
\text { normal camera } \\
\text { height, F geht aus } \\
\text { dem Bild } \\
\text { 3. close-up (F), } \\
\text { Vorwegfahrt, } \\
\text { normal camera } \\
\text { height }\end{array}$ & $\begin{array}{l}\text { beige, orange, } \\
\text { mintgrün/ } \\
\text { oranges Licht } \\
\text { von Lampen } \\
\text { um den } \\
\text { Spiegel/ } \\
\text { dunkle Flure, } \\
\text { künstliches } \\
\text { Licht (Quelle } \\
\text { nicht zu } \\
\text { erkennen) }\end{array}$ & $\begin{array}{l}\text { Spiegel an der } \\
\text { Wand mit Fließen, } \\
\text { im Spiegel sitzt } \\
\text { Aída vor einer } \\
\text { Schminkkommode } \\
\text { mit Spiegel und } \\
\text { Glühbirnen am } \\
\text { Rahmen, } \\
\text { Schminke auf der } \\
\text { Ablage, } \\
\text { F sitzt mit dem } \\
\text { Rücken zum } \\
\text { Spiegel und } \\
\text { wartet/ hört die } \\
\text { Musik, Fgeht } \\
\text { durch dunkle } \\
\text { Flure } \\
\text { (Holzwände), } \\
\text { schmerzverzertes } \\
\text { Gesicht teilweise } \\
\text { ganzim Schatten, }\end{array}$ \\
\hline
\end{tabular}




\begin{tabular}{|c|c|c|c|c|c|c|c|c|}
\hline & & & & & & & & $\begin{array}{l}\text { teilweise mit Licht } \\
\text { bedeckt }\end{array}$ \\
\hline 69 & $\begin{array}{l}1.12 .37- \\
1.12 .52\end{array}$ & Bühne & $\begin{array}{l}\text { F, Aída, Publikum } \\
\text { elegante Kleidung } \\
\text { (high society) }\end{array}$ & $\begin{array}{l}\text { F steht neben der } \\
\text { Bühne, Aída lässt } \\
\text { sich vom Publikum } \\
\text { feiern }\end{array}$ & $\begin{array}{l}\text { Aída: } \\
\text { Klaviermusik } \\
\text { (Lied: Sirena), } \\
\text { Applaus }\end{array}$ & $\begin{array}{l}\text { 1. full shot (Aída, } \\
\text { Publikum), F steht } \\
\text { am Rand der Bühne } \\
\text { imDunkeln (beide } \\
\text { v. hinten), Fbewegt } \\
\text { sich langsam aus } \\
\text { dem Bild }\end{array}$ & beige & $\begin{array}{l}\text { prunkvoller } \\
\text { Konzertsaal, wie } \\
\text { Opernhaus, mit } \\
\text { goldenen } \\
\text { Ornamenten } \\
\text { verzierte Galerien }\end{array}$ \\
\hline 70 & $\begin{array}{l}1.12 .53- \\
1.14 .19\end{array}$ & $\begin{array}{l}\text { Auto, Straße } \\
\text { in Lima }\end{array}$ & F, Aída, Sohn & $\begin{array}{l}\text { Aída und Sohn } \\
\text { freuen sich über } \\
\text { ihren Erfolg, F } \\
\text { lächelt: "les gustó } \\
\text { no?". Aída (wirftF } \\
\text { bösen Blick zu) } \\
\text { fühltsich bedroht } \\
\text { und lässt sie } \\
\text { aufgrund ihres } \\
\text { Kommentars } \\
\text { mitten auf der } \\
\text { Straße aussteigen, } \\
\text { damitbricht sieihr } \\
\text { Versprechen } \\
\text { (Perlen), F zögert } \\
\text { mit verängstigten, } \\
\text { entgeisterten } \\
\text { Blick, dann steigt } \\
\text { sie doch aus, F } \\
\text { rennt und schreit }\end{array}$ & $\begin{array}{l}\text { Motor- } \\
\text { geräusche, } \\
\text { Straßen- } \\
\text { geräusche, F's } \\
\text { Schreie }\end{array}$ & $\begin{array}{l}\text { 1. close-up (Aída), } \\
\text { normal camera } \\
\text { height/ subj. } \\
\text { Kamera (Persp. F) } \\
\text { 2. close-up (F), } \\
\text { normal camera } \\
\text { height } \\
\rightarrow \text { Wdh. } 1 \\
\text { 3. close-up (Sohn, } \\
\text { v. hinten), normal } \\
\text { camera height } \rightarrow \\
\text { mehrmaligeWdh. } 1 \\
+2 \\
\text { 4. medium long } \\
\text { shot (F), subj. } \\
\text { Kamera (Persp. } \\
\text { Aída) }\end{array}$ & $\begin{array}{l}\text { schwarz } \\
\text { (Dunkelheit), } \\
\text { Straßen- } \\
\text { laternen }\end{array}$ & $\begin{array}{l}\text { Auto von innen, } \\
\text { Straße, Lichter } \\
\text { und Häuser durch } \\
\text { die Autofenster }\end{array}$ \\
\hline \multicolumn{9}{|c|}{4 Sekunden Schwarzblende } \\
\hline 71 & $\begin{array}{l}1.14 .25- \\
1.15 .16\end{array}$ & $\begin{array}{l}\text { Wohnraum } \\
\text { des Onkels } \\
\text { und seiner } \\
\text { Familie }\end{array}$ & $\begin{array}{l}\text { Frauen, Frisöre } \\
\text { (ohne } \mathrm{F} \rightarrow \\
\text { Parallelhandlung: } \\
71 \text { und } 72 \text { ) }\end{array}$ & $\begin{array}{l}\text { Frauen werden } \\
\text { von transsexuellen } \\
\text { Frisören für die } \\
\text { Hochzeit gerichtet }\end{array}$ & $\begin{array}{l}\text { Stimmen- } \\
\text { gewirr }\end{array}$ & $\begin{array}{l}\text { 1. medium long } \\
\text { shot (Frauen, } \\
\text { Frisöre, Kinder), } \\
\text { normal camera } \\
\text { height, statische } \\
\text { Kamera }\end{array}$ & $\begin{array}{l}\text { beige, braun/ } \\
\text { Tageslicht von } \\
\text { draußen }\end{array}$ & $\begin{array}{l}\text { Wohnraum: } \\
\text { Stühle und Bilder, } \\
3 \text { Reihen: hinten } \\
\text { transsexuelle } \\
\text { Frisöre, mittig: } \\
\text { Frauen, vorne: }\end{array}$ \\
\hline
\end{tabular}




\begin{tabular}{|c|c|c|c|c|c|c|c|c|}
\hline & & & & & & & & $\begin{array}{l}\text { Kinder und } \\
\text { Kameramann }\end{array}$ \\
\hline 72 & $\begin{array}{l}1.15 .17- \\
1.15 .54\end{array}$ & A. & $\begin{array}{l}\text { F, Tío, Leichnam } \\
\text { F im Nachthemd, } \\
\text { offene Haare } \\
\text { tío: Hemd und } \\
\text { Krawatte }\end{array}$ & $\begin{array}{l}\text { Fentschuldigtsich } \\
\text { bei ihrem Onkel, } \\
\text { er ist sprachlos }\end{array}$ & $\begin{array}{l}\text { Stimmen vom } \\
\text { Hof, Luftholen } \\
\text { (tío) }\end{array}$ & $\begin{array}{l}\text { 1. close-up (tío, } \\
\text { Leichnam), high } \\
\text { shot, F tritt ins Bild }\end{array}$ & $\begin{array}{l}\text { weiß, türkis, } \\
\text { beige/ } \\
\text { Tageslicht von } \\
\text { draußen }\end{array}$ & $\begin{array}{l}\text { Bett mit } \\
\text { mumifiziertem } \\
\text { Leichnam } \\
\text { (himmelblaues } \\
\text { Leichentuch) }\end{array}$ \\
\hline 73 & $\begin{array}{l}1.15 .55- \\
1.16 .12\end{array}$ & $\begin{array}{l}\text { Straße in } \\
\text { Bergen }\end{array}$ & $\begin{array}{l}\text { Hochzeits- } \\
\text { gesellschaft, } \\
\text { Gäste, Verwandte, } \\
\text { F }\end{array}$ & $\begin{array}{l}\text { Familie fährt zur } \\
\text { Hochzeit }\end{array}$ & fröhliche Musik & $\begin{array}{l}\text { 1. long shot } \\
\text { (Familie in } \\
\text { vorbeifahrenden } \\
\text { Rikschas), statische } \\
\text { Kamera }\end{array}$ & $\begin{array}{l}\text { rosa, weiß, } \\
\text { beige/ } \\
\text { Tageslicht }\end{array}$ & $\begin{array}{l}\text { Berge und Straße, } \\
\text { bunte Rikschas } \\
\text { fahren von rechts } \\
\text { nach links }\end{array}$ \\
\hline 74 & $\begin{array}{l}1.16 .13- \\
1.19 .13\end{array}$ & Manchay & $\begin{array}{l}\text { Hochzeits- } \\
\text { gesellschaft, } \\
\text { Gäste, Verwandte, } \\
\text { F } \\
\text { Máxima: } \\
\text { opulentes } \\
\text { Hochzeitskleid, } \\
\text { extrem langer } \\
\text { Schleier mit rosa } \\
\text { Luftballonen } \\
\text { (Schleier sollte } \\
\text { eigentlich fliegen, } \\
\text { aber er fliegt } \\
\text { nicht) } \\
\text { alle in eleganten } \\
\text { Kleidern, manche } \\
\text { in traditionellen } \\
\text { Quechua- } \\
\text { Trachten, } \\
\text { sogar } F \text { trägt ein } \\
\text { hellblaues, langes }\end{array}$ & $\begin{array}{l}\text { Máximas Hochzeit, } \\
\text { sie ärgert sich, } \\
\text { weil ihr Schleier } \\
\text { nicht fliegt, } \\
\text { Fotoshooting vor } \\
\text { paradiesischer } \\
\text { Leinwand, F } \\
\text { immerabseits, sie } \\
\text { leidet }\end{array}$ & $\begin{array}{l}\text { fröhliche Musik } \\
\text { (Lied: Máxima), } \\
\text { Jubelschreie, } \\
\text { Stepptanz }\end{array}$ & $\begin{array}{l}\text { 1. medium long } \\
\text { shot (Marcos, } \\
\text { Máxima, Tante } \\
\text { u.a.), normal } \\
\text { camera height, } \\
\text { statische Kamera } \\
\text { 2. medium long } \\
\text { shot (Familie und } \\
\text { Brautpaar vor } \\
\text { Leinwand), normal } \\
\text { camera height, F } \\
\text { steht am Ende } \\
\text { allein vor der } \\
\text { Leinwand } \\
\text { 3. close shot (F, } \\
\text { tanzende Gäste im } \\
\text { Hintergrund), } \\
\text { Kamera folgt F's } \\
\text { Bewegung, ohne } \\
\text { Schnitt, close-up } \\
\text { (F), Vorwegfahrt,F } \\
\text { geht aus Bild, } \\
\text { Kamera bleibt }\end{array}$ & $\begin{array}{l}\text { sandfarben, } \\
\text { blau, rosa, } \\
\text { weiß/ } \\
\text { Tageslicht bis } \\
\text { Dämmerung (1 } \\
\text { Tag) }\end{array}$ & $\begin{array}{l}\text { Berge und } \\
\text { Armenviertel im } \\
\text { Hintergrund, } \\
\text { kitschige } \\
\text { Hochzeits- } \\
\text { dekoration, } \\
\text { paradiesische } \\
\text { Leinwand für } \\
\text { Fotoshoot, } \\
\text { dahinter Baustelle }\end{array}$ \\
\hline
\end{tabular}




\begin{tabular}{|c|c|c|c|c|c|c|c|c|}
\hline & & & $\begin{array}{l}\text { Tüll-Kleid, ist } \\
\text { geschminkt und } \\
\text { hat Locken }\end{array}$ & & & $\begin{array}{l}\text { stehen, medium } \\
\text { long shot (im Kreis } \\
\text { tanzendeGäste) } \\
\text { 4. close shot } \\
\text { (Ballon), Kamera } \\
\text { verfolgt Ballon, low } \\
\text { camera }\end{array}$ & & \\
\hline 75 & $\begin{array}{l}1.19 .14- \\
1.20 .23\end{array}$ & $\begin{array}{l}\text { Eine Art } \\
\text { Klassen- } \\
\text { zimmer in } \\
\text { Manchay }\end{array}$ & $\begin{array}{l}\text { F, schlafende } \\
\text { Frauen, Tío }\end{array}$ & $\begin{array}{l}\text { F und andere } \\
\text { Frauen schlafen, } \\
\text { Onkel hält Fden } \\
\text { Mund zu, er will } \\
\text { beweisen, dass sie } \\
\text { leben will, sie } \\
\text { wehrt sich und } \\
\text { flüchtet }\end{array}$ & $\begin{array}{l}\text { ruhige } \\
\text { romantische } \\
\text { Musik, F'sNach- } \\
\text { Luft- } \\
\text { schnappen, } \\
\text { schnelle } \\
\text { Schritte, } \\
\text { Schluchzen (tío) }\end{array}$ & $\begin{array}{l}\text { 1. full shot (F, } \\
\text { andere Frauen), } \\
\text { normal camera } \\
\text { height, statische } \\
\text { Kamera } \\
\text { 2. close-up (F's } \\
\text { Gesicht), normal } \\
\text { camera height, tío } \\
\text { nähert sich v. } \\
\text { hinten, F entfernt } \\
\text { sich aus dem Bild, } \\
\text { close-up (tío) }\end{array}$ & türkis, blau & $\begin{array}{l}\text { Klassenzimmer, } \\
\text { Frauen liegen auf } \\
\text { dem Boden, } \\
\text { Tischen, Stühlen, } \\
\text { Leitsprüche („,un } \\
\text { Peru que estudia } \\
\text { es un Peru que } \\
\text { triunfa") und } \\
\text { Plakate an den } \\
\text { Wänden, Tafel mit } \\
\text { Matherechnungen }\end{array}$ \\
\hline 76 & $\begin{array}{l}1.20 .24- \\
1.20 .49\end{array}$ & Manchay & $\begin{array}{l}\text { F } \\
\text { F: blaues Kleid, } \\
\text { offene Haare mit } \\
\text { Locken }\end{array}$ & F rennt & $\begin{array}{l}\text { Gitarren- } \\
\text { musik }\end{array}$ & $\begin{array}{l}\text { 1. close shot }(F) \text {, } \\
\text { Nebenherfahrt, } \\
\text { normal camera } \\
\text { height } \\
\text { 2. medium long } \\
\text { shot }(F), \text { Kamera } \\
\text { auf Position, F } \\
\text { rennt entlang des } \\
\text { Bildes, statische } \\
\text { Kamera }\end{array}$ & $\begin{array}{l}\text { sandfarben, } \\
\text { blau, rot, } \\
\text { steingrau/ } \\
\text { Dunkelheit, } \\
\text { Morgen- } \\
\text { dämmerung }\end{array}$ & $\begin{array}{l}\text { lange Mauer aus } \\
\text { Ziegelsteinen, } \\
\text { darauf steht in } \\
\text { großen, roten } \\
\text { Buchstaben "algo } \\
\text { nuevo está } \\
\text { surgiendo en } \\
\text { Manchay" (der } \\
\text { Rest istnicht } \\
\text { lesbar), } \\
\text { Armenviertel bei } \\
\text { Morgen- } \\
\text { dämmerung }\end{array}$ \\
\hline 77 & $\begin{array}{l}1.20 .50- \\
1.21 .19\end{array}$ & $\begin{array}{l}\text { Weg zum } \\
\text { Herrenhaus, } \\
\text { Markt (Lima) }\end{array}$ & F, Marktarbeiter & $\begin{array}{l}\text { F geht zügig und } \\
\text { entschlossen (aber } \\
\text { weinend) zum } \\
\text { Herrenhaus }\end{array}$ & $\begin{array}{l}\text { Gitarren- } \\
\text { musik, } \\
\text { Stimmen-gewirr }\end{array}$ & $\begin{array}{l}\text { 1. medium shot }(F) \text {, } \\
\text { Hinterherfahrt, } \\
\text { normal camera } \\
\text { height }\end{array}$ & $\begin{array}{l}\text { blau, grün, rot/ } \\
\text { Tageslicht }\end{array}$ & $\begin{array}{l}\text { enge Gassen auf } \\
\text { dem Markt, } \\
\text { Stände an den } \\
\text { Seiten, }\end{array}$ \\
\hline
\end{tabular}




\begin{tabular}{|c|c|c|c|c|c|c|c|c|}
\hline & & & $\begin{array}{l}\text { F: blaues Kleid, } \\
\text { offene Haare mit } \\
\text { Locken } \\
\text { Marktarbeiter in } \\
\text { Schürzen und } \\
\text { Arbeitskleidern }\end{array}$ & & & $\begin{array}{l}\text { 2. close shot (F) } \\
\text { Vorwegfahrt } \\
\text { 3. medium shot } \\
\text { (Markt/ } \\
\text {-verkäufer) subj. } \\
\text { Kamera (Persp. F) } \\
\text { 4. close shot (F), } \\
\text { Nebenherfahrt } \\
\rightarrow \text { Wdh. } 2+3+4, \\
\text { Kamera bleibt } \\
\text { stehen, F geht aus } \\
\text { dem Bild }\end{array}$ & & $\begin{array}{l}\text { Fleischwaren und } \\
\text { andere } \\
\text { Lebensmittel, } \\
\text { Labyrinth }\end{array}$ \\
\hline 78 & $\begin{array}{l}1.21 .20- \\
1.22 .21\end{array}$ & $\mathrm{~F}$. & $\begin{array}{l}\text { F, Aída } \\
\text { F: blaues Kleid, } \\
\text { offene Haare mit } \\
\text { Locken } \\
\text { Aída: man sieht } \\
\text { nurihren Arm, der } \\
\text { wie von einer } \\
\text { Toten leblos } \\
\text { herabhängt }\end{array}$ & $\begin{array}{l}\text { Fschleichtsichins } \\
\text { Schlafzimmer, sie } \\
\text { sieht sich wieder } \\
\text { im Bild des } \\
\text { Offiziers, sammelt } \\
\text { Perlen vom Boden } \\
\text { (kriecht wie ein } \\
\text { scheues Tier), wird } \\
\text { im Garten } \\
\text { ohnmächtig }\end{array}$ & $\begin{array}{l}\text { Gitarren- } \\
\text { musik (leiser } \\
\text { werdend), F's } \\
\text { stockender } \\
\text { Atem, Vogelge- } \\
\text { zwitscher, } \\
\text { Schritte, Öffnen } \\
\text { des } \\
\text { Eingangstors }\end{array}$ & $\begin{array}{l}\text { 1. extreme close-up } \\
\text { (Bild Offizier, F's } \\
\text { Spiegelbild darin), F } \\
\text { nähert sich } \\
\text { 2. close-up (F, } \\
\text { Perlen, Arm Aída), F } \\
\text { kriecht in das Bild, } \\
\text { normal camera } \\
\text { height } \\
\text { 3. extrem close-up } \\
\text { (F's Gesicht), } \\
\text { Vorwegfahrt }\end{array}$ & $\begin{array}{l}\text { steingrau, } \\
\text { mintgrün, } \\
\text { blau/ Morgen- } \\
\text { dämmerung/ } \\
\text { Tageslicht }\end{array}$ & $\begin{array}{l}\text { F im Spiegelbild } \\
\text { des Bildes vom } \\
\text { Offizier, Bett von } \\
\text { der Seite, } \\
\text { Teppichboden, } \\
\text { Perlen sind auf } \\
\text { dem Boden } \\
\text { verstreut und } \\
\text { führen zu Aída's } \\
\text { Arm (als hätte sie } \\
\text { die Perlen in der } \\
\text { Hand gehabt) }\end{array}$ \\
\hline 79 & $\begin{array}{l}1.22 .22- \\
1.24 .17\end{array}$ & C. & F, Noé & $\begin{array}{l}\text { Noé findet } F, \\
\text { bedeckt ihre Brust } \\
\text { und tröstet sie, } F \\
\text { weint und will die } \\
\text { Kartoffel } \\
\text { entfernen lassen }\end{array}$ & $\begin{array}{l}\text { Kirchenglocke, } \\
\text { Fahrrad fällt auf } \\
\text { den Boden, } \\
\text { schnelle } \\
\text { Schritte, } \\
\text { Stimmen- } \\
\text { gewirr, Vogelge- } \\
\text { zwitscher }\end{array}$ & $\begin{array}{l}\text { 1. extreme close-up } \\
\text { (F's Hand mit } \\
\text { Perlen), normal } \\
\text { camera height } \\
\text { 2. close-up (F, } \\
\text { Noé), high shot, } \\
\text { Kamera bewegt } \\
\text { sich mit }\end{array}$ & $\begin{array}{l}\text { grün, blau, } \\
\text { steingrau/ } \\
\text { Tageslicht }\end{array}$ & $\begin{array}{l}\text { Fliegtaufdem } \\
\text { Steinboden am } \\
\text { Eingangstor }\end{array}$ \\
\hline 80 & $\begin{array}{l}1.24 .18- \\
1.24 .38\end{array}$ & $\begin{array}{l}\text { Markt/ } \\
\text { Straße Lima }\end{array}$ & F, Noé & $\begin{array}{l}\text { Noé trägt F auf } \\
\text { dem Rückenzum } \\
\text { Krankenhaus }\end{array}$ & $\begin{array}{l}\text { Stimmen- } \\
\text { gewirr, hupende } \\
\text { Autos }\end{array}$ & $\begin{array}{l}\text { 1. close-up (F auf } \\
\text { Noé's Rücken), } \\
\text { Nebenherfahrt }\end{array}$ & $\begin{array}{l}\text { bunt/ } \\
\text { Tageslicht }\end{array}$ & $\begin{array}{l}\text { enge Gassen auf } \\
\text { dem Markt, große } \\
\text { Fleischwaren }\end{array}$ \\
\hline
\end{tabular}




\begin{tabular}{|c|c|c|c|c|c|c|c|c|c|}
\hline & & & & & & & $\begin{array}{l}\text { 2. close shot } \\
\text { (beide), } \\
\text { Nebenherfahrt }\end{array}$ & & $\begin{array}{l}\text { hängen an den } \\
\text { Ständen }\end{array}$ \\
\hline 81 & $\begin{array}{l}1.24 .39- \\
1.25 .09\end{array}$ & $\begin{array}{l}\text { Krankenhaus } \\
\text { Lima, Flur }\end{array}$ & $\begin{array}{l}\text { F, Noé, } \\
\text { Angehörige von } \\
\text { Patienten, } \\
\text { Krankenhaus- } \\
\text { personal }\end{array}$ & $\begin{array}{l}\text { Noé trägt F zur } \\
\text { Notaufnahme }\end{array}$ & & $\begin{array}{l}\text { schnelle } \\
\text { Schritte, } \\
\text { hallender Flur }\end{array}$ & $\begin{array}{l}\text { 1. medium shot } \\
\text { (beide), } \\
\text { Vorwegfahrt, Noé } \\
\text { bewegt sich aus } \\
\text { dem Bild }\end{array}$ & $\begin{array}{l}\text { weiß, blau/ } \\
\text { Tageslicht von } \\
\text { draußen }\end{array}$ & $\begin{array}{l}\text { kahler } \\
\text { Krankenhausflur } \\
\text { mit Fenstern, } \\
\text { ohne Schilder, } \\
\text { ohne Sitzmöglich- } \\
\text { keiten, ein } \\
\text { Informations- } \\
\text { Glaskasten }\end{array}$ \\
\hline 82 & $\begin{array}{l}1.25 .10- \\
1.25 .52\end{array}$ & $\begin{array}{l}\text { Kranken- } \\
\text { haus, } \\
\text { Behand- } \\
\text { lungs- } \\
\text { zimmer }\end{array}$ & $\begin{array}{l}\text { F, Tío } \\
\text { F: nackt, aber } \\
\text { zugedeckt mit } \\
\text { einer beigen } \\
\text { Decke }\end{array}$ & $\begin{array}{l}\text { F wacht auf, Onkel } \\
\text { sitzt bei ihr, F zeigt } \\
\text { Perlen in der Hand }\end{array}$ & & $\begin{array}{l}\text { Klimpern der } \\
\text { Perlen }\end{array}$ & $\begin{array}{l}\text { 1. close-up } \\
\text { (Neonlampe), } \\
\text { extreme high shot } \\
\text { 2. close-up }(F) \text {, } \\
\text { subj. Kamera } \\
\text { (Persp. Tío) } \\
\text { 3. full shot (F,tío), } \\
\text { normal camera } \\
\text { height } \\
\text { 4. close-up (F's } \\
\text { Hand mit Perlen, } \\
\text { tío's Handum F's) } \\
\text { 5. close-up }(F), \\
\text { subj. Kamera } \\
\text { (Persp. Tío) }\end{array}$ & weiß, beige & $\begin{array}{l}\text { grelle, runde } \\
\text { Neonlampe an der } \\
\text { Decke, F liegt auf } \\
\text { einer Trage in } \\
\text { einem kahlen, } \\
\text { sterilen Raum, der } \\
\text { teilweise mit } \\
\text { einem Vorhang } \\
\text { geschlossen ist }\end{array}$ \\
\hline 83 & $\begin{array}{l}1.25 .53- \\
1.27 .43\end{array}$ & $\begin{array}{l}\text { Straße zur } \\
\text { Küste }\end{array}$ & $\begin{array}{l}\text { F, Familie, } \\
\text { Leichnam } \\
\text { Alltagskleidung }\end{array}$ & $\begin{array}{l}\text { Familie fährt im } \\
\text { Pickup mit dem } \\
\text { Leichnam, F } \\
\text { entdeckt das } \\
\text { Meer, Fwill } \\
\text { anhalten }\end{array}$ & $\begin{array}{l}\text { Meer } \\
\text { Mumie }\end{array}$ & $\begin{array}{l}\text { Fahrtwind, } \\
\text { Motor- } \\
\text { geräusche, } \\
\text { Rauschen der } \\
\text { Wellen, Klopfen } \\
\text { auf Pickup-Dach }\end{array}$ & $\begin{array}{l}\text { 1. extremelong } \\
\text { shot(Straße, } \\
\text { Gebirge, Boot), } \\
\text { Kamerafahrt/ subj. } \\
\text { Kamera (Persp. F) } \\
\text { 2. medium shot } \\
\text { (Familie auf } \\
\text { Ladefläche), normal } \\
\text { camera height }\end{array}$ & $\begin{array}{l}\text { steingrau, } \\
\text { sandfarben, } \\
\text { blau/Tageslicht }\end{array}$ & $\begin{array}{l}\text { lange Straße ohne } \\
\text { Ende, Berge drum } \\
\text { herum, vereinzelt } \\
\text { Schilder am } \\
\text { Straßenrand, } \\
\text { fahren durch } \\
\text { einen Tunnel im } \\
\text { Berg, davor steht } \\
\text { ein großen Holz- } \\
\text { Boot auf einem }\end{array}$ \\
\hline
\end{tabular}




\begin{tabular}{|c|c|c|c|c|c|c|c|c|c|}
\hline & & & & & & & $\begin{array}{l}\text { 3. close-up (F), } \\
\text { normal camera } \\
\text { height, im } \\
\text { Hintergrund das } \\
\text { Meer } \\
\text { 4. extreme long } \\
\text { shot (Pick-up zw. } \\
\text { Sanddünen) }\end{array}$ & & $\begin{array}{l}\text { Anhänger (passt } \\
\text { nicht durch den } \\
\text { Tunnel } \rightarrow \\
\text { Anspielung auf } \\
\text { Kartoffel?), } \\
\text { Sanddünen und } \\
\text { Meer } \\
\text { (verschwommen) }\end{array}$ \\
\hline 84 & $\begin{array}{l}1.27 .44- \\
1.29 .06\end{array}$ & $\begin{array}{l}\text { Küste } \\
\text { (Sanddünen) }\end{array}$ & F, Leichnam & $\begin{array}{l}\text { F trägt den } \\
\text { Leichnam auf dem } \\
\text { Rücken zum Meer, } \\
\text { F singt und } \\
\text { verabschiedet sich } \\
\text { von ihrer Mutter }\end{array}$ & $\begin{array}{l}\text { Meer } \\
\text { Mumie }\end{array}$ & $\begin{array}{l}\text { Rauschen der } \\
\text { Wellen, } \\
\text { Gitarren- } \\
\text { musik (lauter } \\
\text { werdend), Off: } \\
\text { Gesang F } \\
\text { (Quechua) }\end{array}$ & $\begin{array}{l}\text { 1. extreme long } \\
\text { shot ( } F \text { trägt } \\
\text { Leichnam durch } \\
\text { Sanddünen) } \\
\text { 2. close-up ( } F \text { v. } \\
\text { hinten, Meer vor } \\
\text { ihr) } \\
\text { 3. extreme long } \\
\text { shot ( } F \text {, Leichnam, } \\
\text { Meer) }\end{array}$ & $\begin{array}{l}\text { steingrau, } \\
\text { blau/ } \\
\text { Tageslicht }\end{array}$ & $\begin{array}{l}\text { Sanddünen mit } \\
\text { einzelner Straße, } \\
\text { hellblauer Himmel } \\
\text { mit Wolken, Berge } \\
\text { im Hintergrund } \\
\text { (wie direkter } \\
\text { Übergang zw. } \\
\text { Küste und } \\
\text { Bergen), } \\
\text { verlassenerStrand }\end{array}$ \\
\hline \multicolumn{10}{|c|}{11 Sekunden Schwarzblende/ Gitarrenmusik } \\
\hline 85 & $\begin{array}{l}1.29 .18- \\
1.29 .43\end{array}$ & B. & $\begin{array}{l}\text { Kinder } \\
\text { Alltagskleider }\end{array}$ & $\begin{array}{l}\text { Kinder tanzen auf } \\
\text { dem Dach und } \\
\text { rufen } F\end{array}$ & & $\begin{array}{l}\text { Stepptanz der } \\
\text { Kinder, } \\
\text { Hundebellen }\end{array}$ & $\begin{array}{l}\text { 1. full shot (Kinder), } \\
\text { low shot }\end{array}$ & $\begin{array}{l}\text { sandfarben, } \\
\text { bunt/ } \\
\text { Tageslicht }\end{array}$ & $\begin{array}{l}\text { Blick von unten } \\
\text { aufunfertiges } \\
\text { Dach, nackter } \\
\text { Beton, } \\
\text { Ziegelsteine, } \\
\text { Berge im } \\
\text { Hintergrund }\end{array}$ \\
\hline 86 & $\begin{array}{l}1.29 .44- \\
1.30 .20\end{array}$ & B. & $\begin{array}{l}\text { F } \\
\text { F: Bluse, Kettegut } \\
\text { sichtbar }\end{array}$ & $\begin{array}{l}\text { Föffnet das Tor } \\
\text { und entdeckt } \\
\text { (lächelnd) die } \\
\text { blühende } \\
\text { Kartoffelpflanze, } \\
\text { sie riecht daran }\end{array}$ & $\begin{array}{l}\text { Kartoffel- } \\
\text { Pflanze } \\
\text { Taubenkette }\end{array}$ & $\begin{array}{l}\text { Öffnen des Tors, } \\
\text { Stimmen aus } \\
\text { dem Viertel, } \\
\text { Wind }\end{array}$ & $\begin{array}{l}\text { 1. close-up (F), } \\
\text { normal camera } \\
\text { height } \\
\text { 2. extreme close-up } \\
\text { (Kartoffelpflanze, } \\
\text { F's Gesicht), normal } \\
\text { camera height }\end{array}$ & $\begin{array}{l}\text { steingrau, } \\
\text { weiß, grün/ } \\
\text { Tageslicht }\end{array}$ & $\begin{array}{l}\text { dunkles Metall- } \\
\text { Eingangstor, auf } \\
\text { dem Boden davor } \\
\text { Kartoffelpflanze }\end{array}$ \\
\hline
\end{tabular}




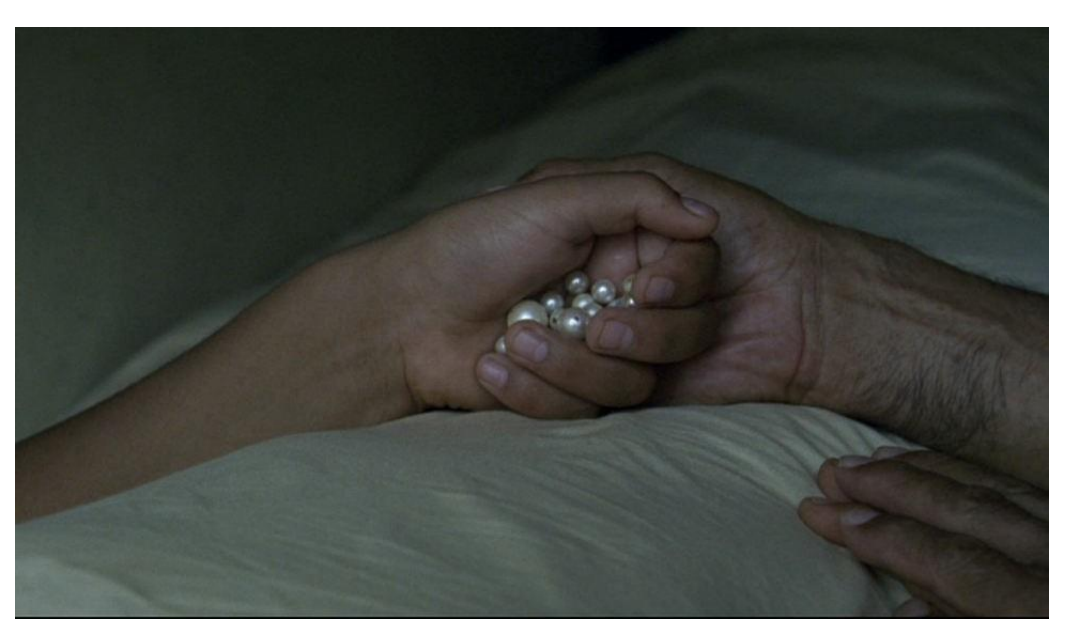

Screenshot Nr. 1

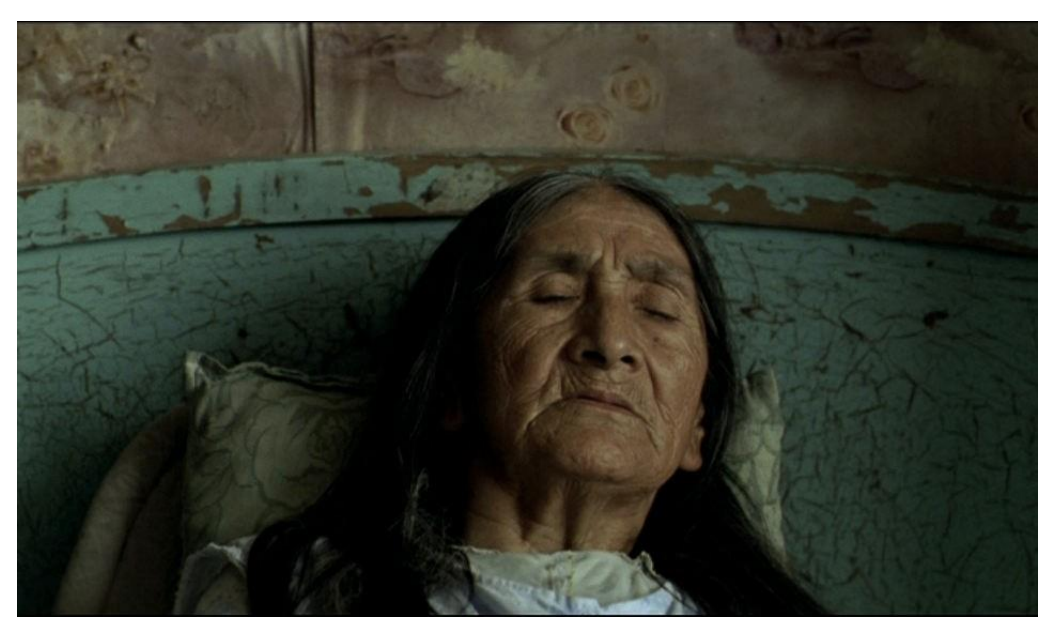

Screenshot Nr. 3

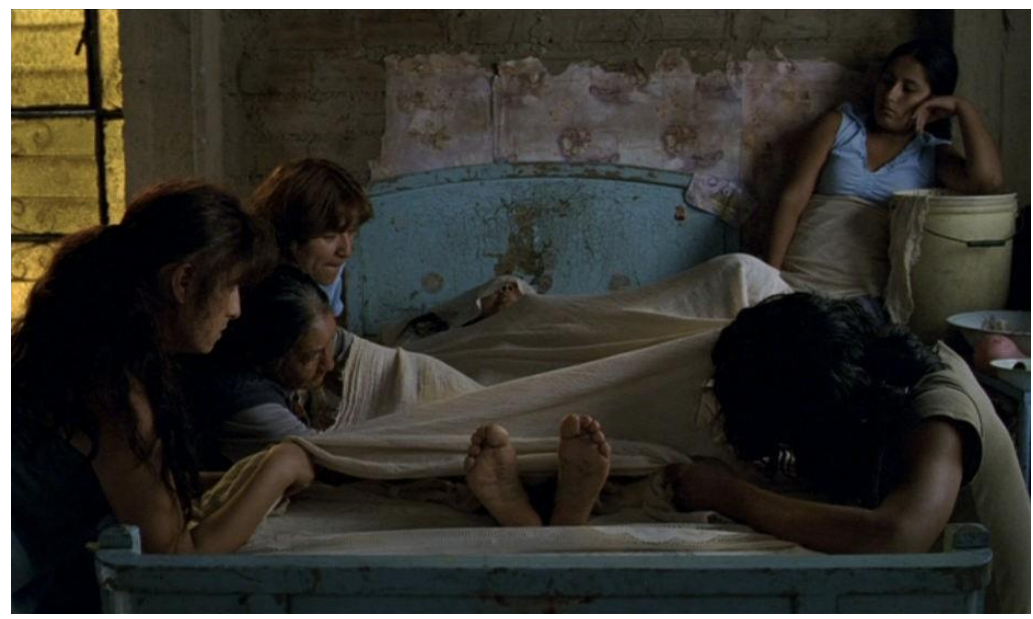

Screenshot Nr. 2

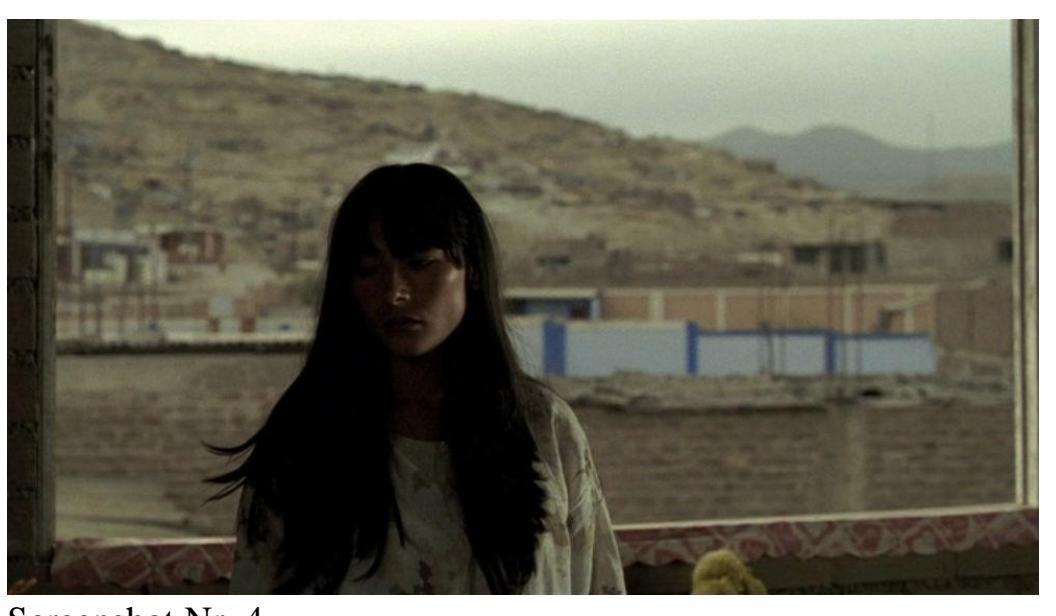

Screenshot Nr. 4 


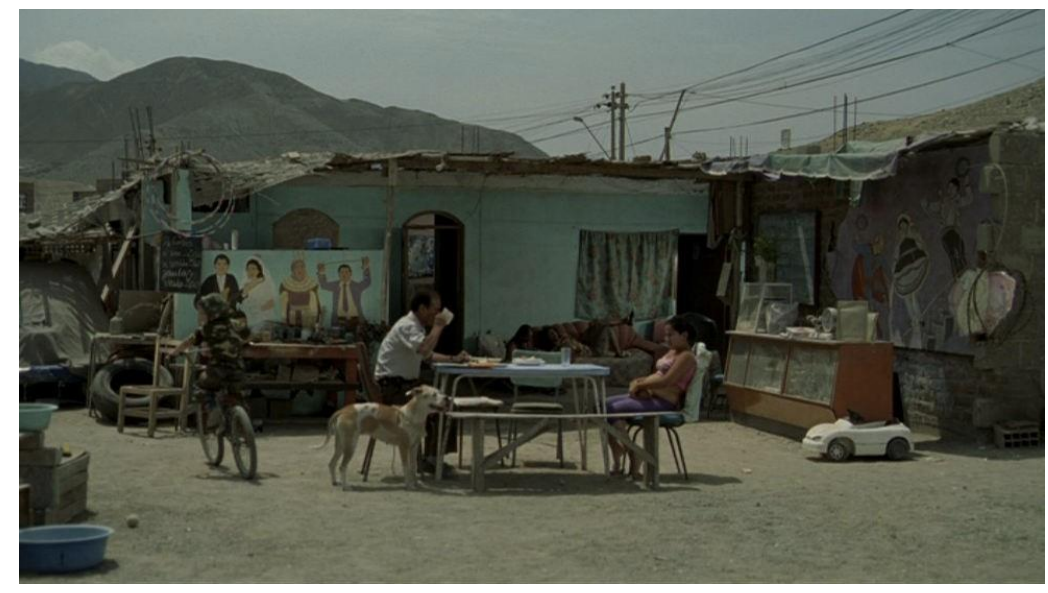

Screenshot Nr. 5

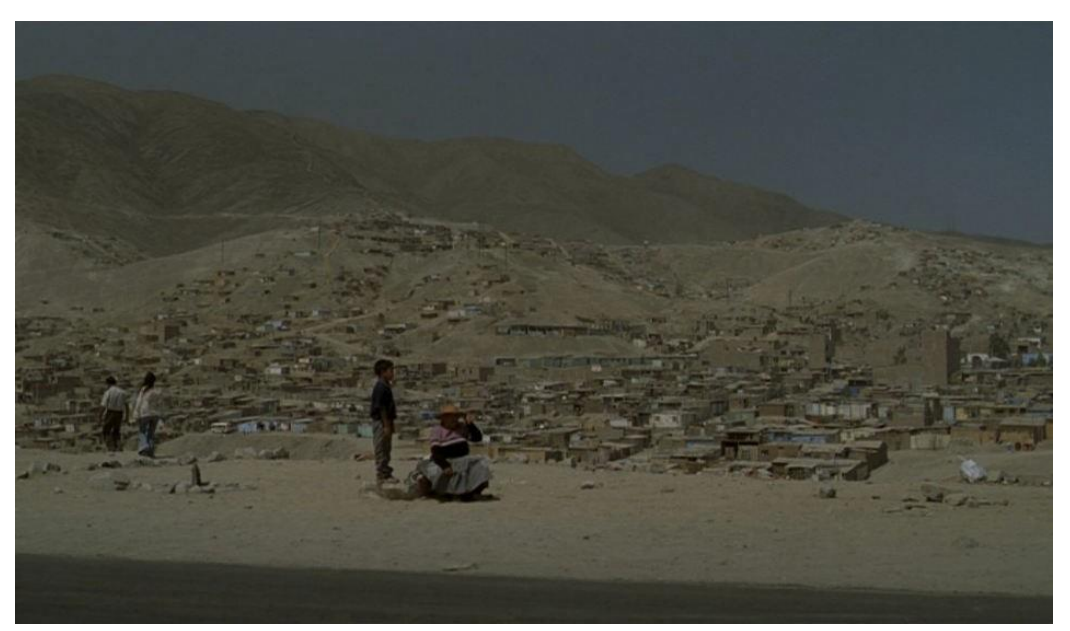

Screenshot Nr. 7

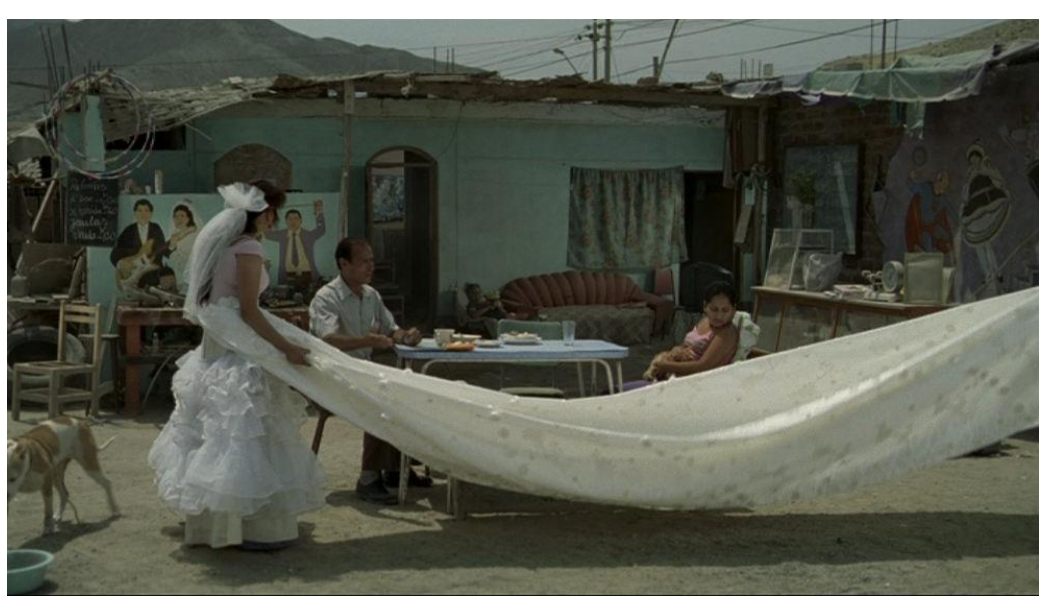

Screenshot Nr. 6

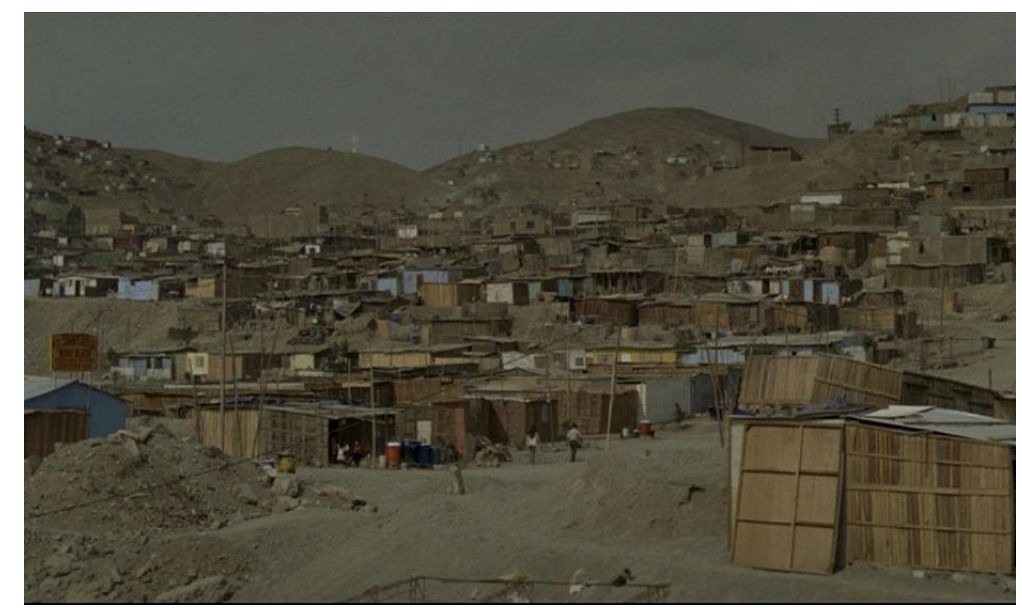

Screenshot Nr. 8 


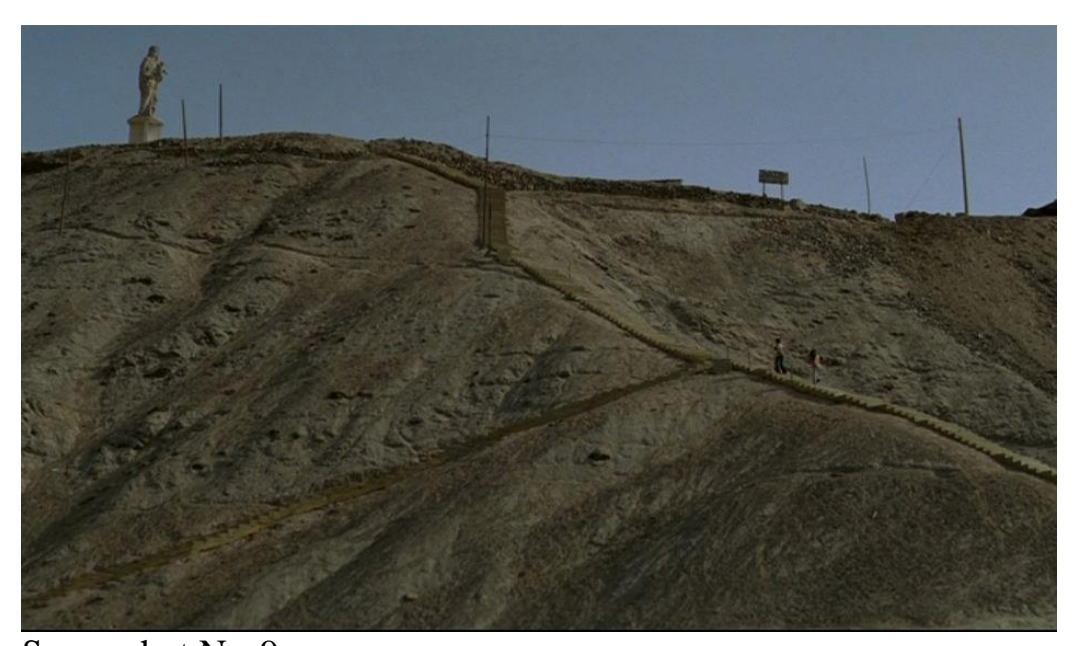

Screenshot Nr. 9

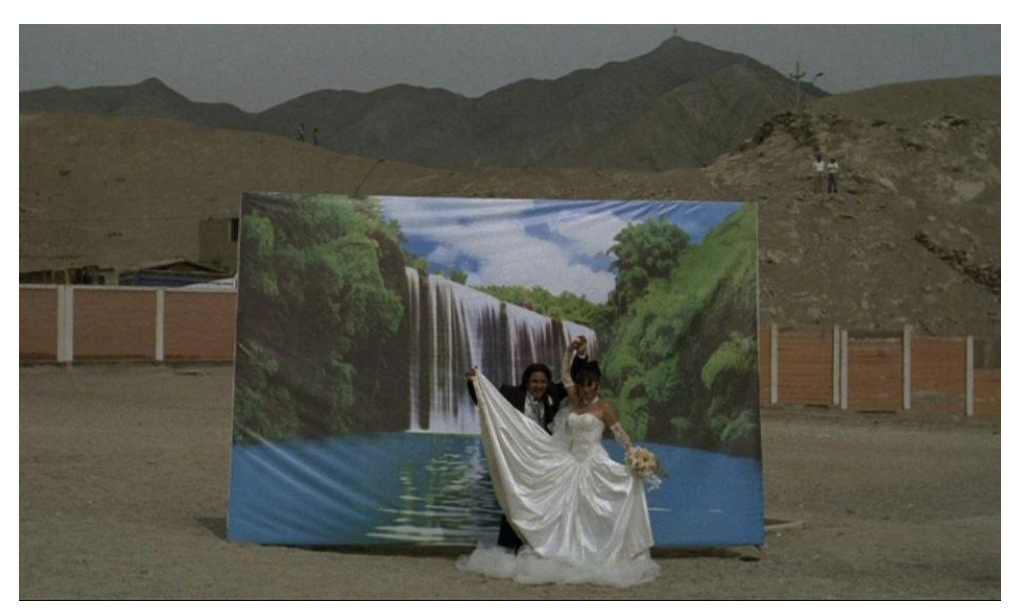

Screenshot Nr. 11

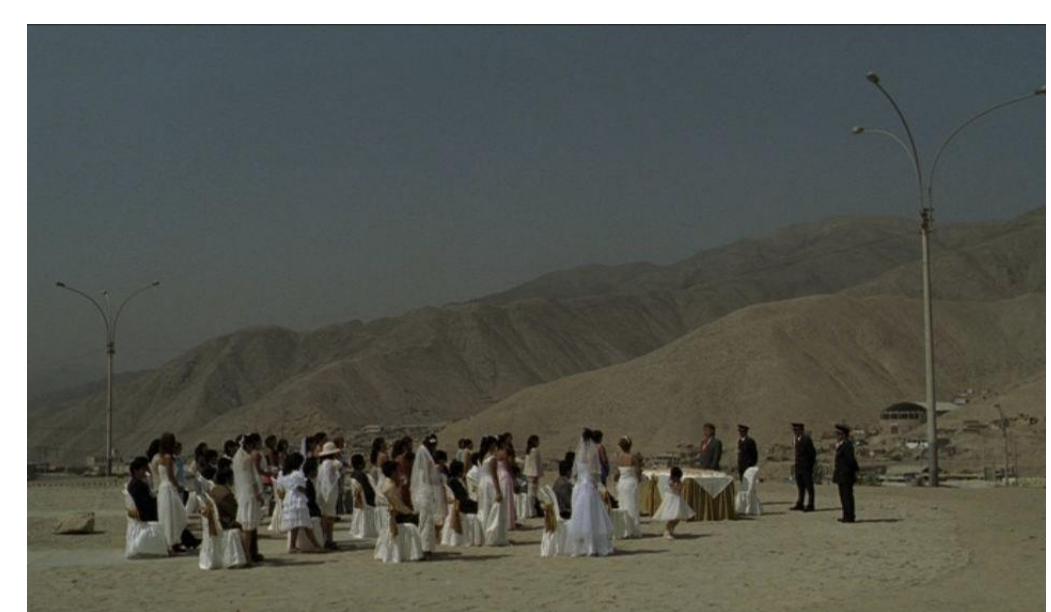

Screenshot Nr. 10

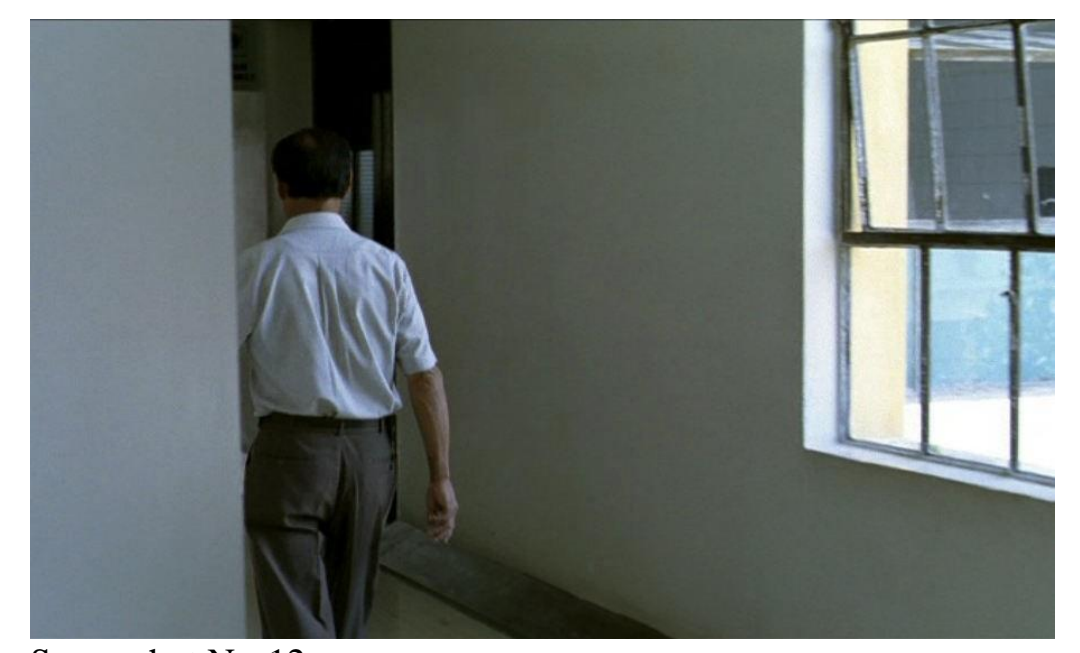

Screenshot Nr. 12 


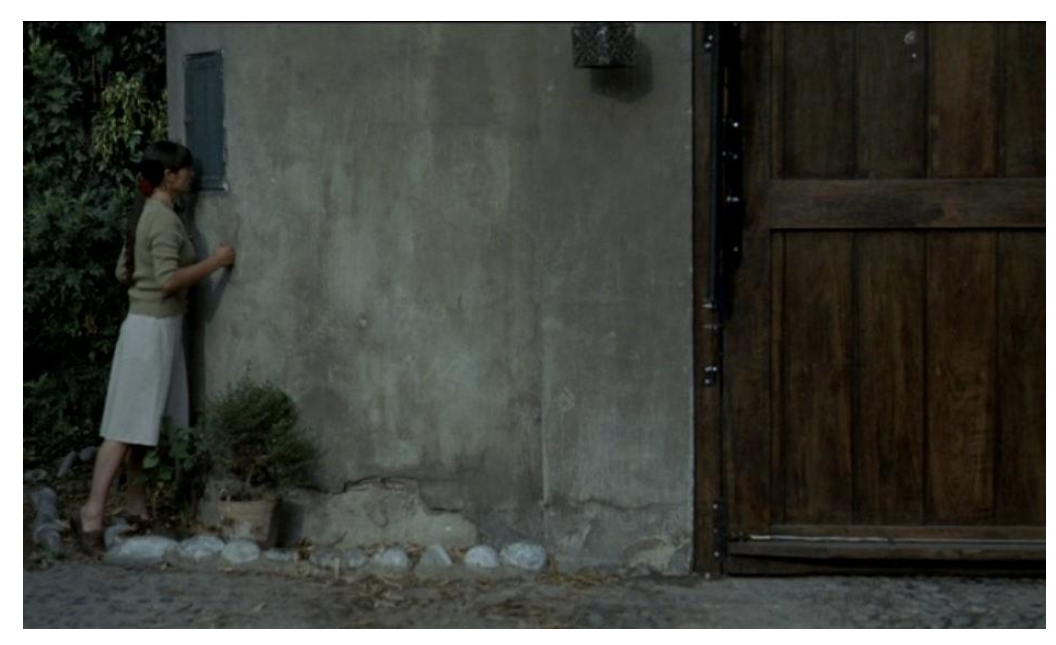

Screenshot Nr. 13

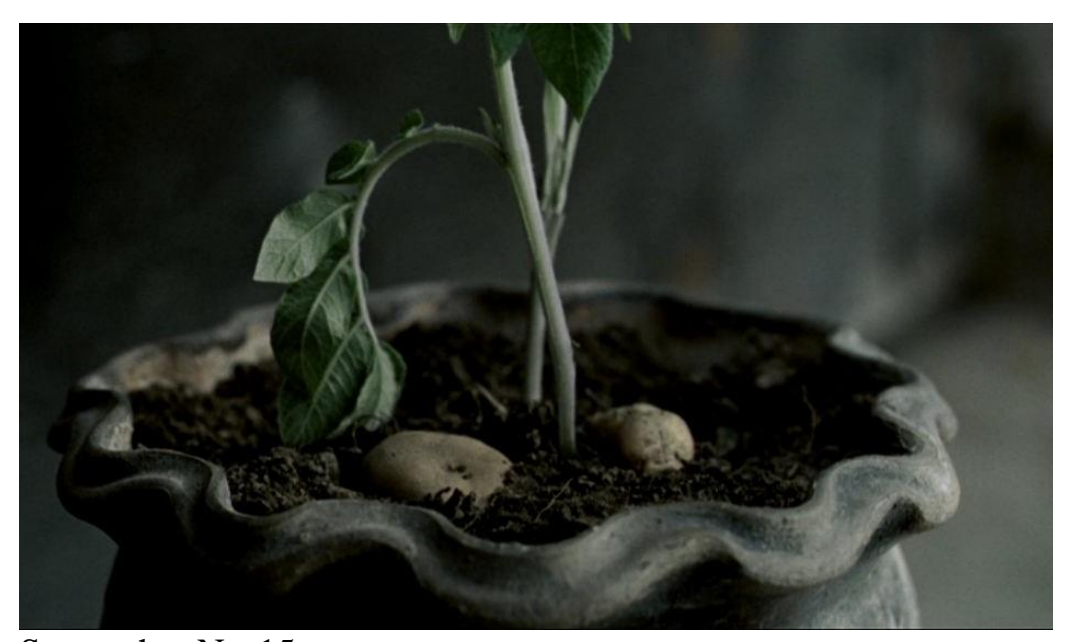

Screenshot Nr. 15

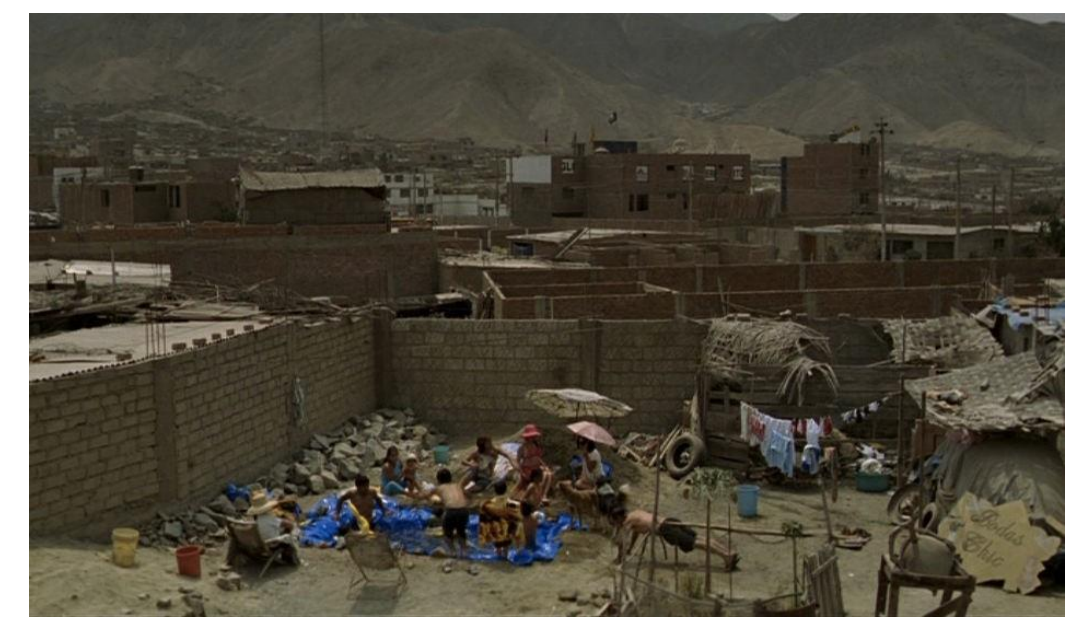

Screenshot Nr. 14

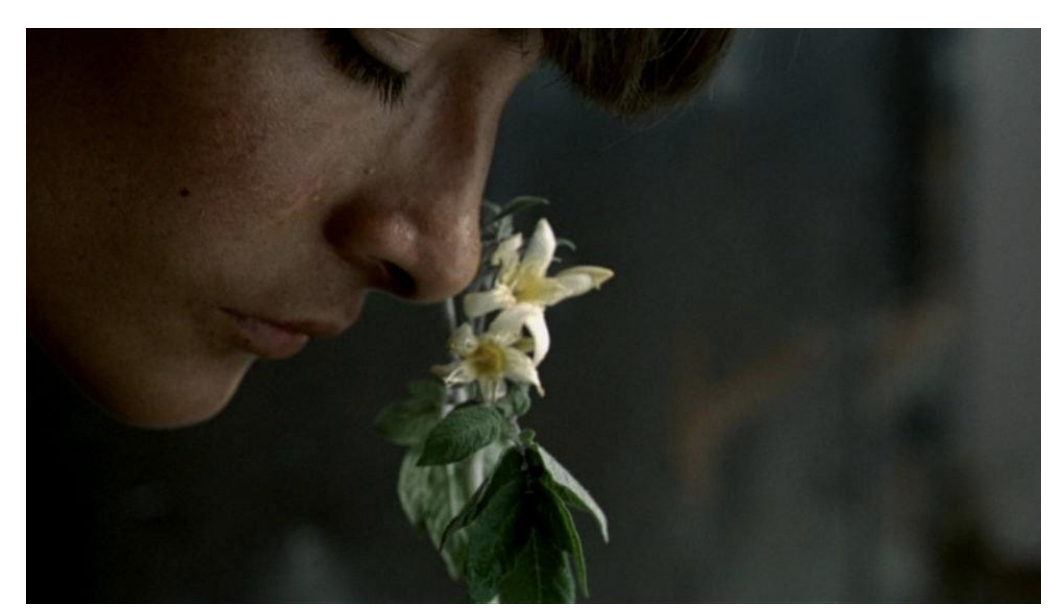

Screenshot Nr. 16 


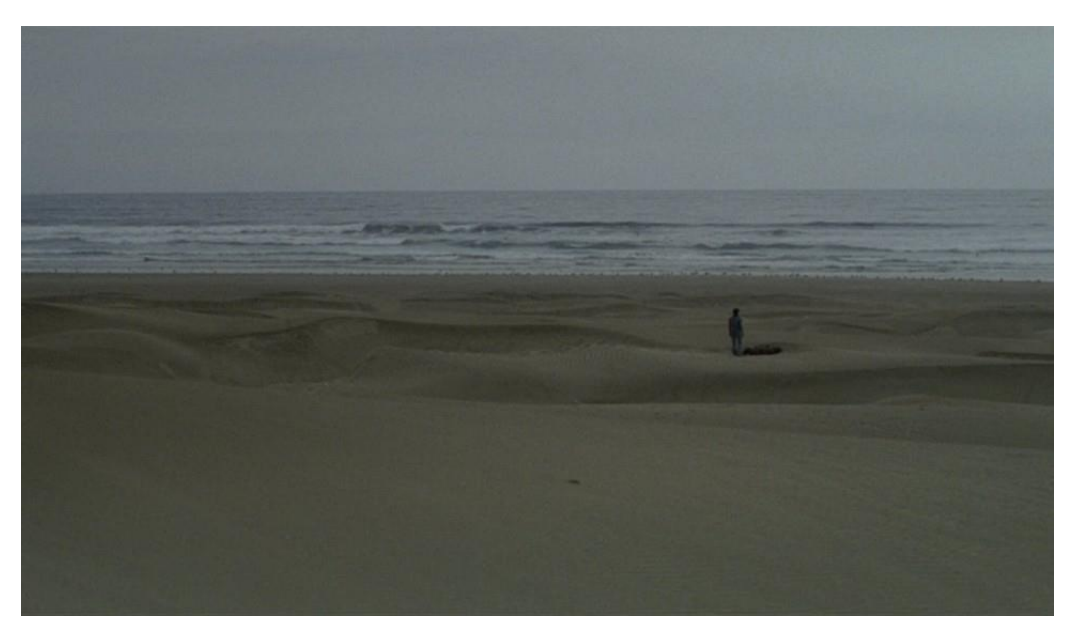

Screenshot Nr. 17

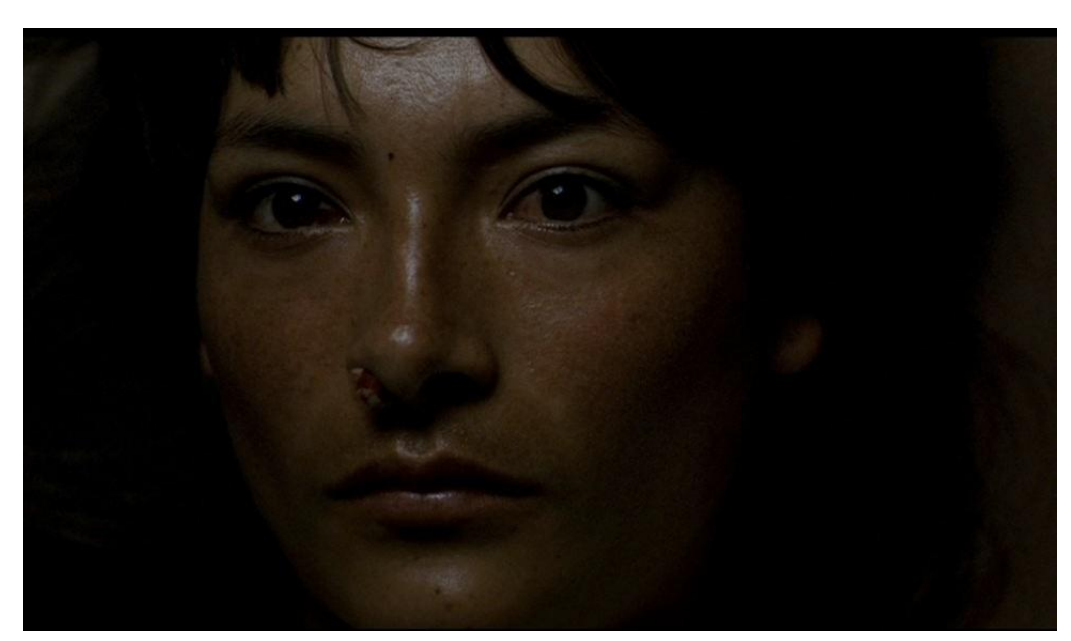

Screenshot Nr. 19

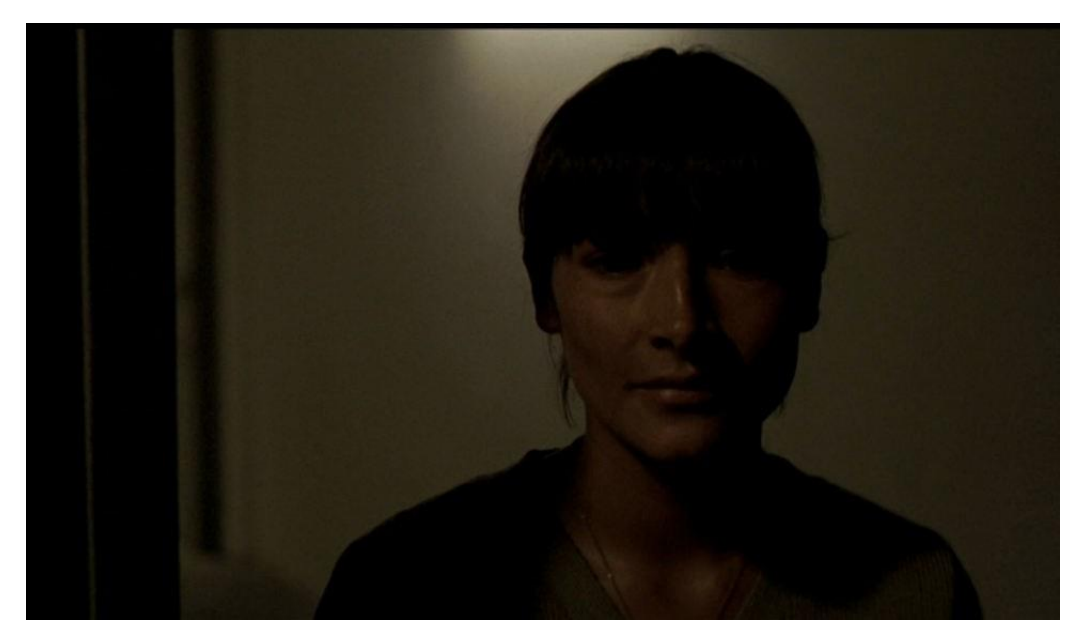

Screenshot Nr. 18

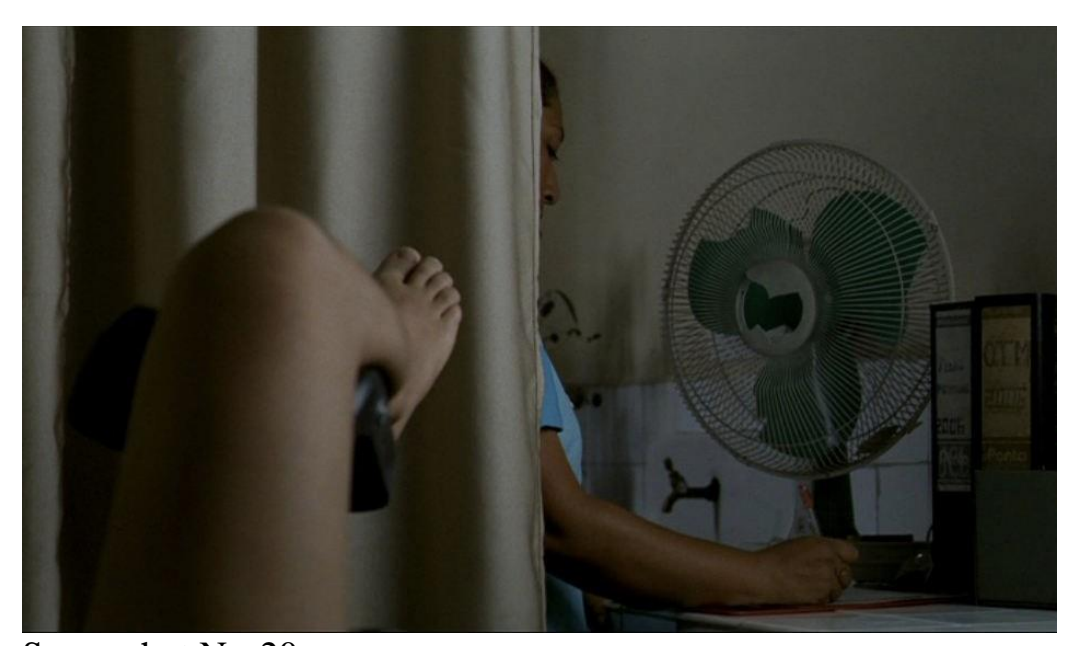

Screenshot Nr. 20 


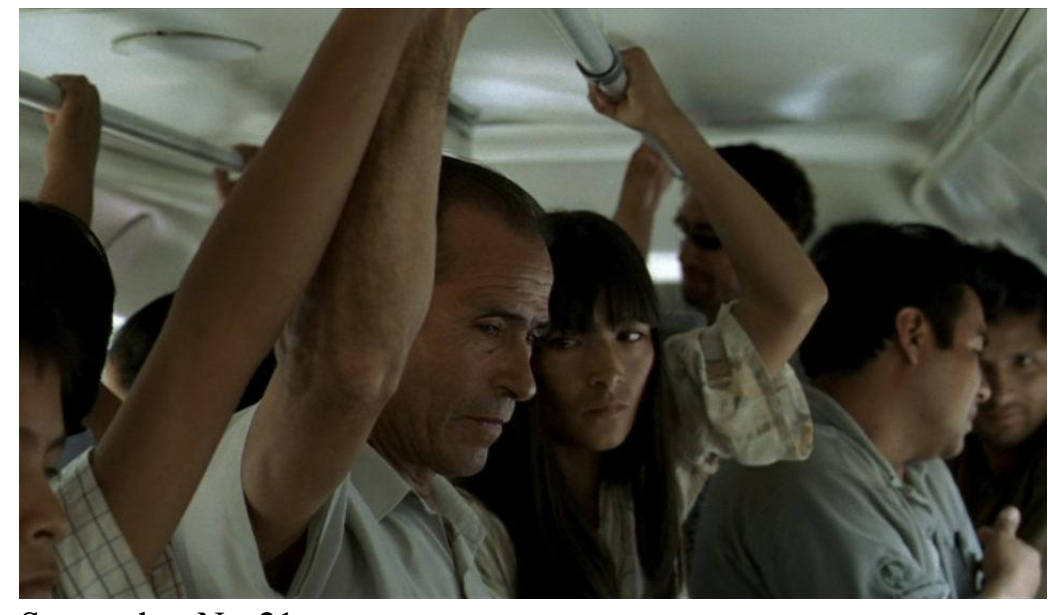

Screenshot Nr. 21

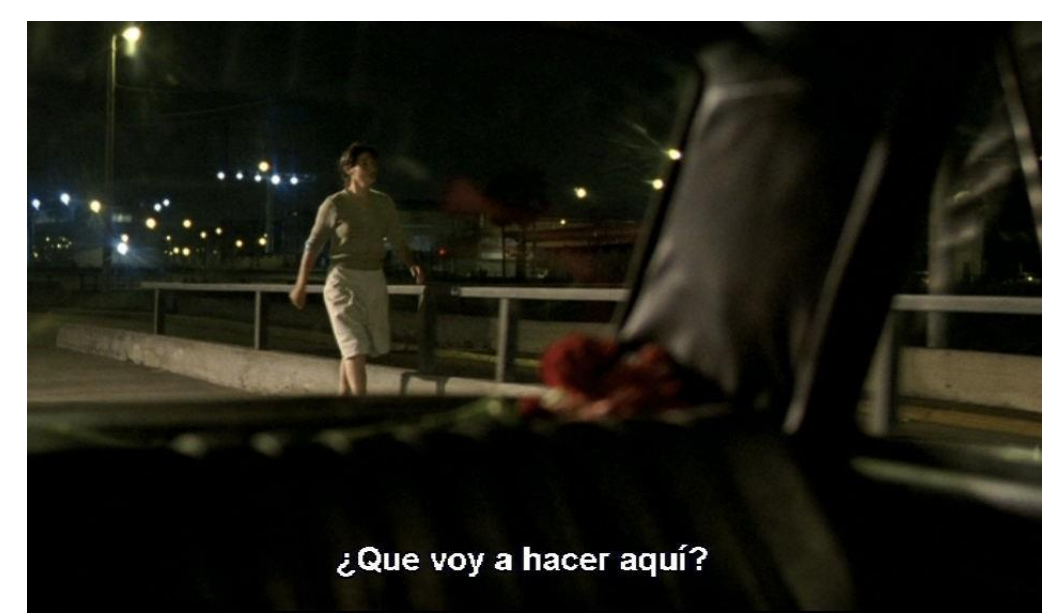

Screenshot Nr. 22 


\section{Eidesstattliche Erklärung}

Ich erkläre, dass ich die Arbeit selbstständig angefertigt und nur die angegebenen Hilfsmittel benutzt habe. Alle Stellen, die dem Wortlaut oder dem Sinn nach anderen Werken, gegebenenfalls auch elektronischen Medien, entnommen sind, sind von mir durch Angabe der Quelle als Entlehnung kenntlich gemacht. Entlehnungen aus dem Internet sind durch Angabe der Quelle und des Zugriffsdatums sowie dem Ausdruck der ersten Seite belegt; sie liegen zudem für den Zeitraum von 2 Jahren entweder auf einem elektronischen Speichermedium im PDF-Format oder in gedruckter Form vor.

Freiburg, den 30.08.2018

Unterzeichnet von Yasemin Soydan 


\section{manchayperu}

Información desde Manchay al mundo y desde el mundo a Manchay.

$\underline{16 \text { noviembre, } 201616 \text { noviembre, } 2016 \text { por manchayperu }}$

\section{Nosotros}

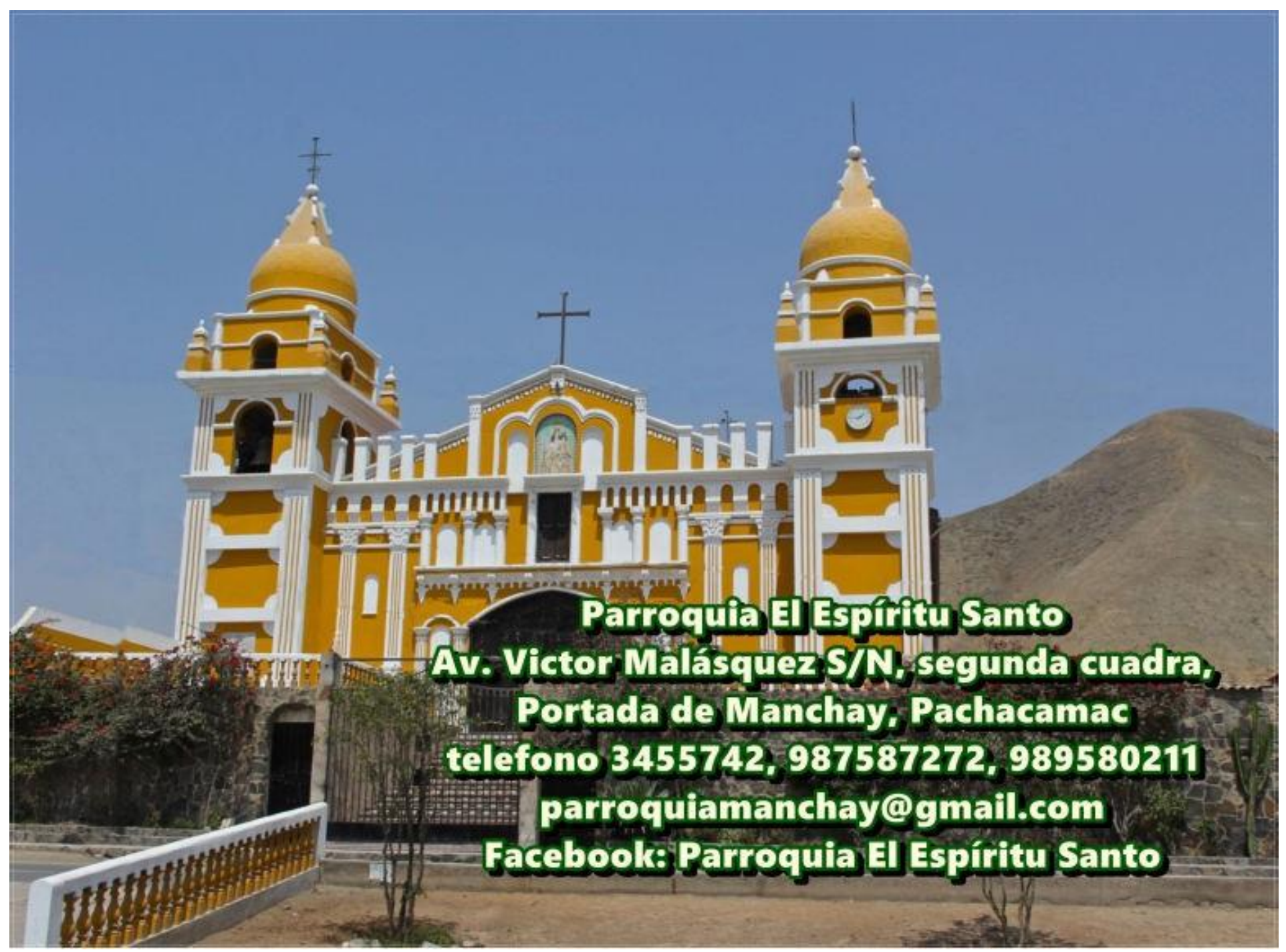

Posted in Super importante

Blog de WordPress.com. 


\section{VashiVisuals}

\section{Average Shot Length}

\section{Revealing Every Single Shot in "Gone Girl” \& "Girl With the Dragon Tattoo" in 1 Image}

\section{DAVID FINCHER FILMS AVERAGE SHOT LENGTH (ASL)}

THE SOCIAL NETWORK (2010)

3.0 seconds

GIRL WITH THE DRAGON TATTOO (2011)

ALIEN 3 (1992)

3.2 seconds

3.6 seconds

GONE GIRL (2014)

3.7 seconds

THE GAME (1997)

3.9 seconds

BENJAMIN BUTTON (2008)

4.0 seconds

SE7EN (1995)

4.5 seconds

ZODIAC (2007)
5.0 seconds
Click to enlarge

CONNECT WITH VASHI

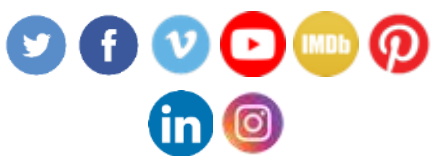

To search type and hit enter..

SUBSCRIBE FOR UPDATES

Email Address*

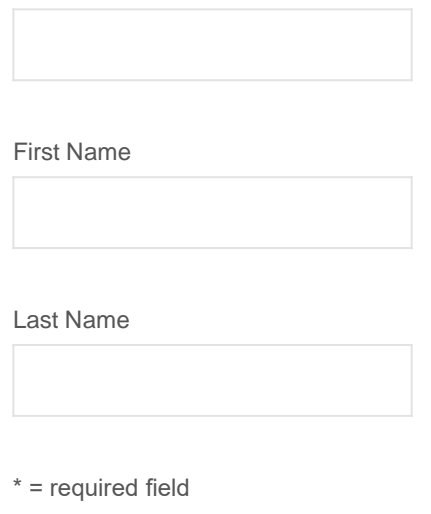

Subscribe

SPEAKING VENUES INCLUDE:

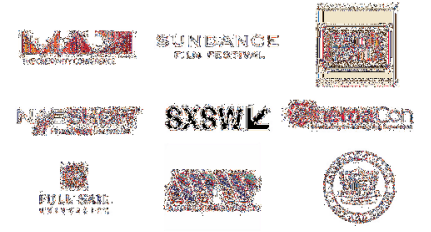

filmmakers and cinephiles further comprehend the mystery behind the numbers.
AVERAGE SHOT LENGTH

Analyzing the average shot length (ASL) of films / TV / music videos can be very telling or completely irrelevant. Taken as its own metric...it is just a number. The supposition that action / thriller / sci-fi films genres have a shorter ASL is statistically accurate but that does not mean a longer ASL means less tension, action, drama or intensity. I have been deep-diving into ASL statistics for several years and shared a lot of SHAREABLES to help

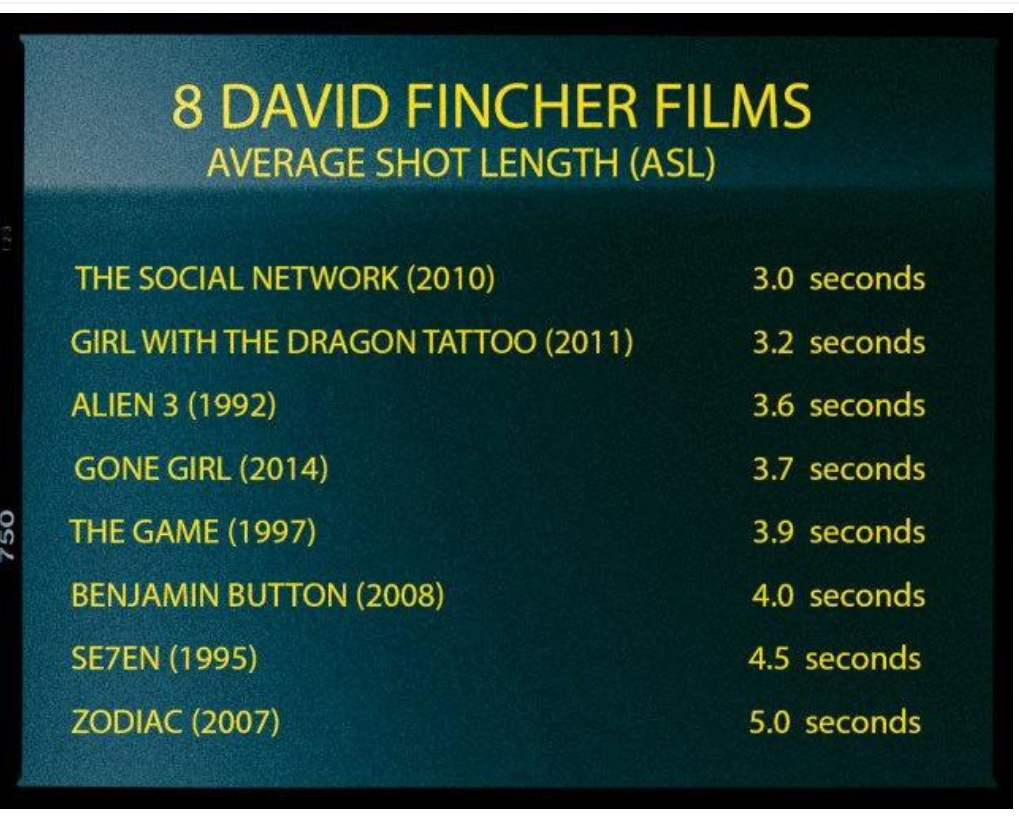


News (https://soundsandcolours.com/news/) Music(https://soundsandcolours.com/music/) Film (https://soundsandcolours.com/!lm//) Arts \&Books(https://soundsandcolours.com/books/) What's On (https://soundsandcolours.com/event)

City Guides:

London (https://soundsandcolours.com/locations/london/), New York (https://soundsandcolours.com/locations/new-york/)

About (https://soundsandcolours.com/about/) Shop (https://soundsandcolours.com/shop/) Subscribe

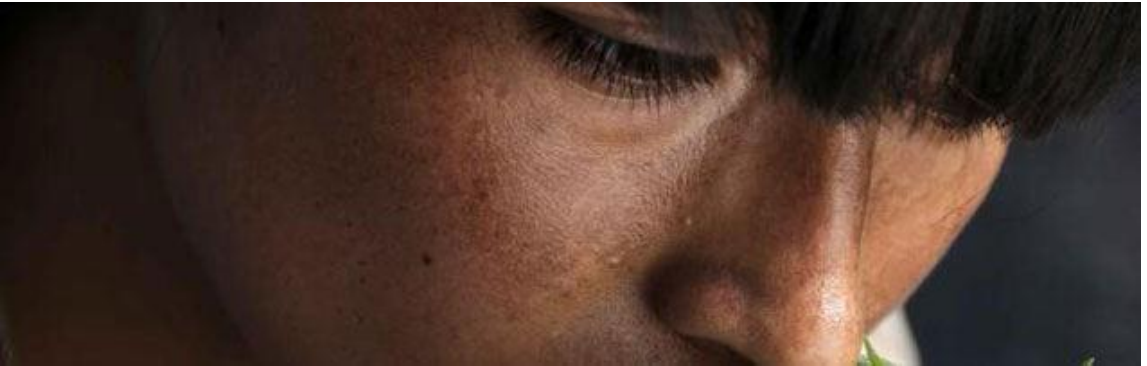

\section{THE MAGIC REALISM OF PERUVIAN CLAUDIA LLOSA'S 'LA TETA ASUSTADA' AND 'MADEINUSA'}

By Leo Nikolaidis (https://soundsandcolours.com/author/leo/) | 04 September, 2010

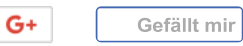

The !lm world rarely publicises women directors, and sometimes, when pressed, it can be hard to think of any at all. For the most part, the !lm director still occupies what Francis Ford Coppola called the 'last truly dictatorial post,' in a world where roles have become increasingly democratic or market driven. Film sets are notoriously chaotic, and for the director to juggle and coordinate so many di"erent aspects - the team of camera operators, stage managers, actors, the time and budget restraints - is perhaps traditionally seen as something which one needs an aggressive man's voice and temper to do. Let the women look pretty and get emotional on screen, leave the technical stu" to us boys.

But some deep and di\#cult !lms have come out of the last 12 months with women in full control, Home by Ursula Meier; Lourdes by Jessica Hausner; even a British o"ering, Fishtank by Andrea Arnold; all refreshingly original in style and content. This should please feminist !lm critics who obsess about the corrupting power of the 'male gaze'; as well as those of us who like emotionally heavy and raw themes to be dealt with in imaginative ways.

La Teta Asustada (The Milk of Sorrow) (2010) from Claudia Llosa, is billed as a magic realist !lm from Peru, but it is actually much cleverer than that. To fully appreciate the magic realist label one must make a distinction from the more fantastical side of the genre. Rather than dazzling the viewer with scary monsters and the fairydust of CGI, Llosa puts the viewer in the same position as the characters with respect to the myth of the !lm's title, 'the milk of sorrow'. Children born in times of great distress or as the product of rape, are said to contract a disease which robs them of a soul.

There are no obvious conclusions to the truth of this tale, we are just forced to interpret what we see, in the light of the story we are given. Here you see no mischievous cartoon demons, just Fausta's awkwardness with everyone, which then perpetuates and reinforces everyone else's suspicious reactions towards her. A black cloud hovers over her, an ever present reminder of the brutal circumstances of her birth.

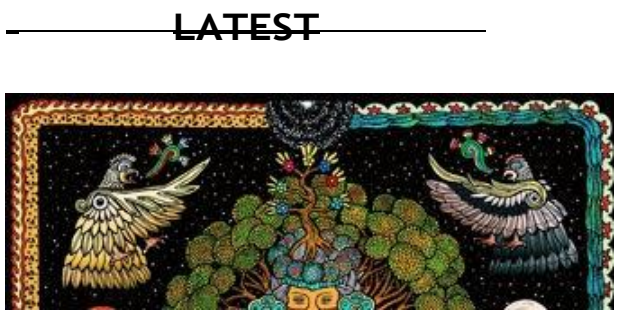

Ecuador

(Https://Soundsandcolours.com/E / REVIEW Huaira- Ñka

Shunku

(Https://Soundsandcolours.com/A Nuka-Shunku-42286/).

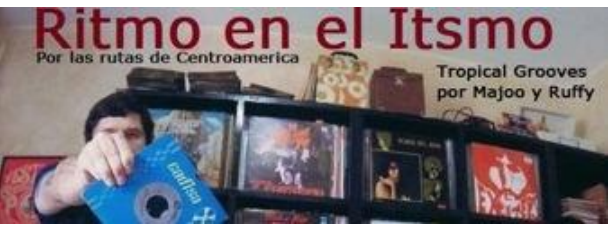

Costa Rica

(Https://Soundsandcolours.com/C

Rica/) / El Salvador

(Https://Soundsandcolours.com/El

Salvador/) / Guatemala

(Https://Soundsandcolours.com/G

I Honduras

(Https://Soundsandcolours.com/H

/ Nicaragua

(Https://Soundsandcolours.com/N

I Panama

(Https://Soundsandcolours.com/P

I Majoo Salguero Y Ruffy's

'Ritmo En El Itsmo' Central

American Mixtape

(Https://Soundsandcolours.com/S

Salguero-Y-Ruffys-Ritmo-

En-El-Itsmo-Central-

American-Mixtape-

42213/) 
$(/)$

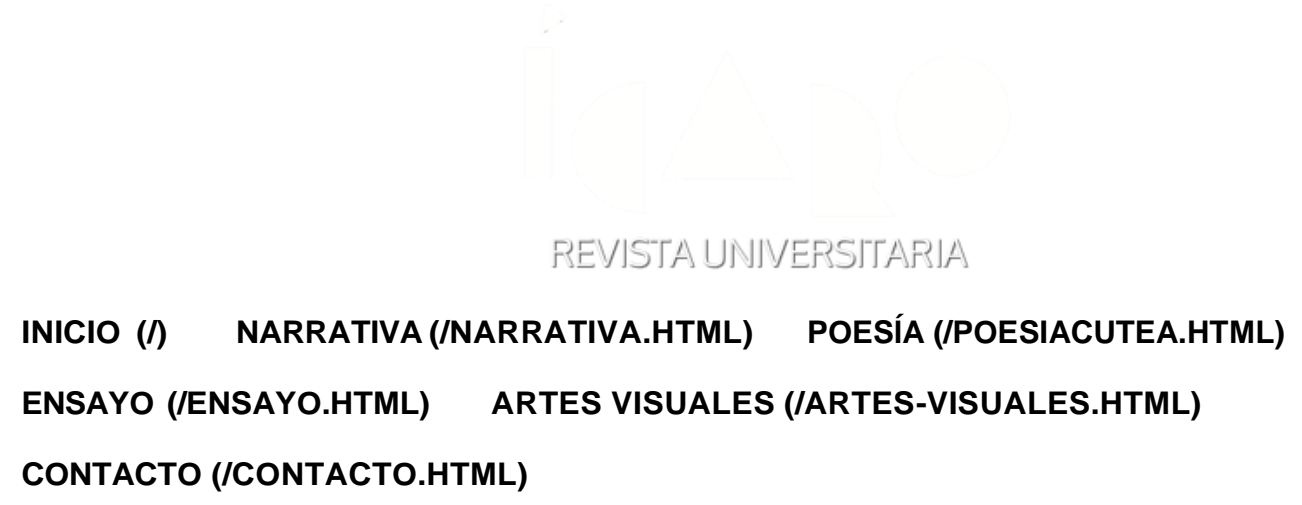

\title{
UN VIAJE AL MIEDO DE LA LIBERTAD: \\ LA TETA ASUSTADA, LO MEJOR DEL NUEVO CINE LATINOAMERICANO
}

POR MARIO GALVÁN REYES

\begin{abstract}
Actualmente el cine latinoamericano pasa por un momento de mucha proyección internacional, pues lejos de ser una fábrica de sueños como en algún tiempo lo fue, ahora busca ser un agente social de cambio, como elemento didáctico que explique la realidad con detalles mágicos de su folclory cultura.

En abril de 1985 se creó la Fundación del Nuevo Cine Latinoamericano (http://www.cinelatinoamericano.org/) bajo la dirección de Gabriel García Márquez, excelso novelista e incansable difusor cultural. Desde entonces ha sido una institución que trabaja en aras de la integración del cine regional, de lograr un universo audiovisual común y de cooperar en la lucha por rescatar y aSanzar la identidad cultural de América Latina y el Caribe. En su base de datos se encuentran registrados 3010 directores, de los cuales su mayoría no rebasan los 38 años de edad. Es decir, existe un medio prolíSco con una oferta cinematográSca variada y realizada por jóvenes directores.

Así, una nueva generación de cineastas ha tomado la responsabilidad de ejercer no sólo un trabajo estético sino ilustrador, de denuncia, que rompe paradigmas estéticos y propone un nuevo lenguaje cinematográSco. Una de las integrantes de esta nueva oleada es Claudia Llosa, joven directora peruana, quien a sus 35 años presume en su haber 2 largometrajes exitosos: Madeinusa (http://youtu.be/ECMTqYw_6IY) y La teta asustada (http://youtu.be/hAxBkfBBTTI), trabajos ganadores de 9 premios y otras menciones en distintos festivales mundialmente reconocidos.
\end{abstract}

A continuación describiré detalladamente los aspectos importantes de La teta asustada; a mi parecer una obra relevante para el contexto actual de América Latina y el medio cinematográSco dado sus valores narrativos, temáticos y estéticos. 
$\underline{\text { Verfasserin der Wissenschaftlichen Arbeit }}$

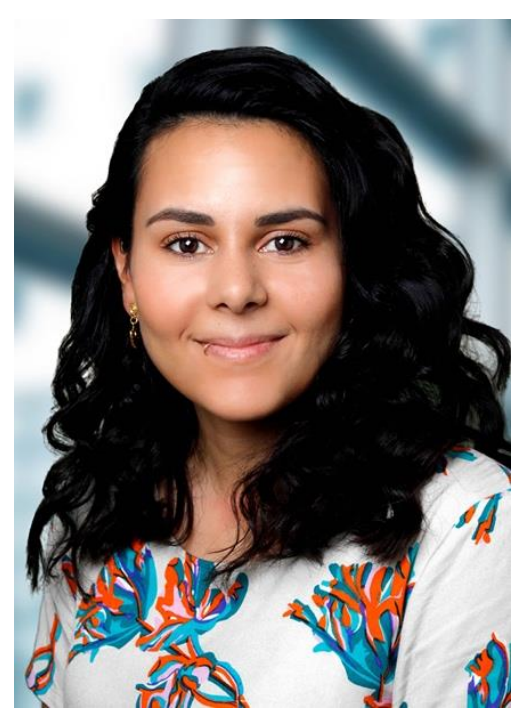

Name: Yasemin Soydan

Geburtstag: 14.06 .1990

Geburtsort: Kehl

Wohnort: Freiburg

Studium: Staatsexamen in Spanisch und Französisch, Albert-Ludwigs-Universität Freiburg

Tätigkeit: Referendarin an einem Beruflichen Gymnasium in Freiburg 